\title{
POSTTRAUMATIC SYMPTOMATOLOGY, INTERPERSONAL RELATIONSHIP FUNCTIONING, AND THEIR ASSOCIATION FOLLOWING TRAUMA EXPOSURE
}

by

\section{Philippe Shnaider}

Master of Arts, Ryerson University, October 2012

Bachelor of Arts, York University, June 2010

\author{
A dissertation presented to Ryerson University \\ in partial fulfillment of the requirements for the degree of \\ Doctor of Philosophy in the Program of Psychology
}

Toronto, Ontario, Canada, 2016

CPhilippe Shnaider 2016 
Author's Declaration for Electronic Submission of a Dissertation

I hereby declare that I am the sole author of this dissertation. This is a true copy of the dissertation, including any required final revisions, as accepted by my examiners.

I authorize Ryerson University to lend this dissertation to other institutions or individuals for the purpose of scholarly research.

I further authorize Ryerson University to reproduce this dissertation by photocopying or by other means, in total or in part, at the request of other institutions or individuals for the purpose of scholarly research.

I understand that my dissertation may be made electronically available to the public. 


\title{
Posttraumatic Symptomatology, Interpersonal Relationship Functioning, and their Association Following Trauma Exposure
}

Doctor of Philosophy 2016

Philippe Shnaider

Psychology

Ryerson University

\begin{abstract}
Interpersonal relationship functioning problems have been documented among individuals with posttraumatic stress disorder (PTSD). Although the trajectory of PTSD following traumatization is well understood, there is minimal research examining the trajectory of interpersonal relationship functioning following trauma. It is yet to be determined if interpersonal relationship functioning confers risk/resilience for PTSD, if interpersonal relationship functioning problems are a consequence of PTSD, or if the direction of the association between these constructs changes over time. The current study examined the trajectory of PTSD and interpersonal relationship functioning, following recent trauma exposure, and the effects that these constructs exert on one another over time. Trauma-exposed individuals $(N=107)$ and their close significant others (e.g., intimate partner, family member, close friend) were recruited from the community. Trauma-exposed individuals completed clinician-administered and self-report measures of PTSD, as well as a self-report measure of interpersonal relationship functioning in reference to their relationship with their close significant other. Four assessments took place at 4-month intervals. PTSD severity declined over time. The trajectory of self-reported, but not clinicianassessed, PTSD was associated with end-state PTSD. Relationship support and depth deteriorated over time. After controlling for trauma type, relationship support and depth
\end{abstract}


worsened over time as end-state PTSD symptom severity improved. Significant associations between initial levels of interpersonal relationship functioning and end-state PTSD were detected, in both bivariate models and in the context of interaction terms. Initial relationship conflict was positively associated with end-state PTSD among participants participating with intimate close others but not those participating with non-intimate close others. There was a negative association between initial relationship support and end-state PTSD among those who experienced non-interpersonal traumas, and a positive association between initial relationship depth and end-state PTSD among those who experienced interpersonal traumas. Findings did not support the hypothesis that the direction of the association between interpersonal relationship functioning and PTSD changes over time. Results can inform early intervention efforts aimed at reducing risk for PTSD following trauma. Brief interpersonally-based interventions, which could be "indicated" based on the type of trauma exposure and whether someone is in an intimate relationship, should be considered and further investigated. 


\section{Acknowledgements}

I would like to thank my supervisor, Dr. Candice M. Monson for her continued support throughout this process. The lessons she has taught me over the past 6 years will continue to shape who I am both personally and professionally. I would also like to extend my gratitude to Dr. Michael K. Suvak (The Wolf) for solving problems, his patience, and the countless hours of statistical help. I would like to thank Dr. Maya Roth for her insightful feedback and for always findings ways to involve me in her work. Finally, I would like to thank Dr. Elizabeth McCay and Dr. Ruth A. Lanius for their willingness to contribute their time. 
Table of Contents

Author's Declaration for Electronic Submission of a Dissertation ............................................ ii

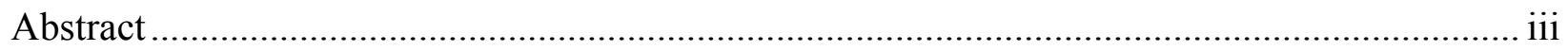

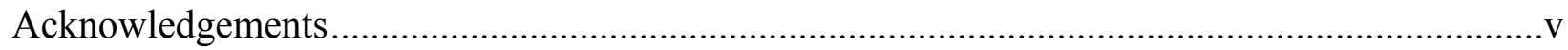

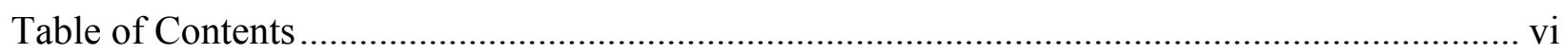

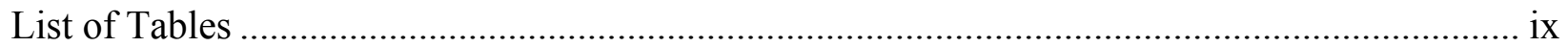

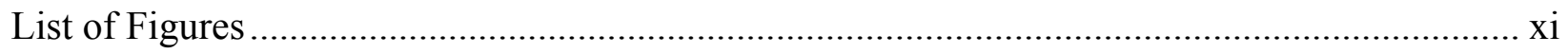

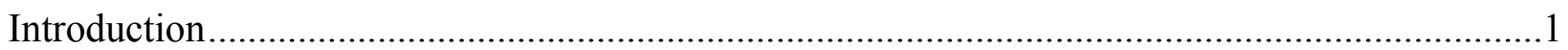

Trauma Exposure and Posttraumatic Stress Disorder...................................................2

Interpersonal Relationship Functioning and Posttraumatic Stress Disorder.....................4

Interpersonal Relationship Dysfunction as an Outcome of Posttraumatic Stress

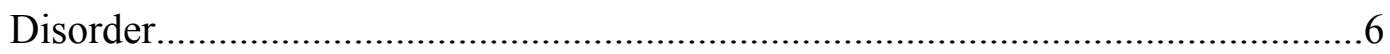

Interpersonal Relationship Functioning as a Risk/Resilience Factor for

Posttraumatic Stress Disorder ...............................................................

The Association between Interpersonal Relationship Functioning and

Posttraumatic Stress Disorder as Bidirectional and Time-dependent....................9

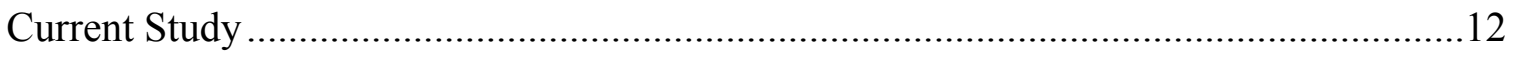

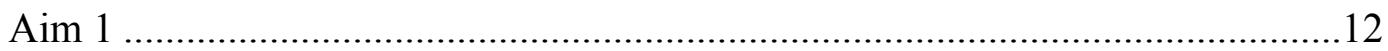

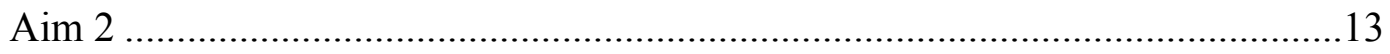

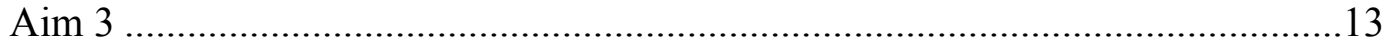

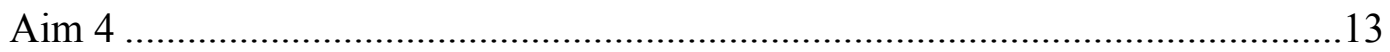

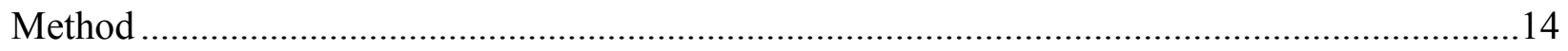

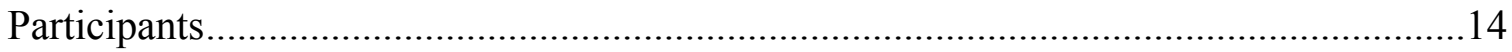




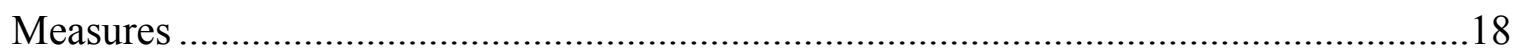

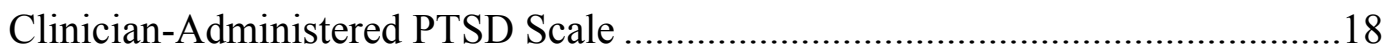

MINI International Neuropsychiatric Interview ...............................................19

Mental Health Care Utilization ......................................................................20

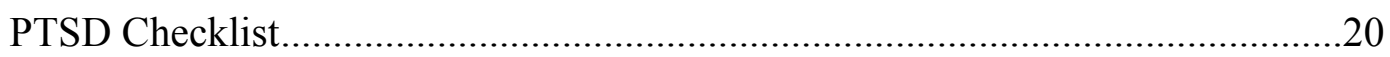

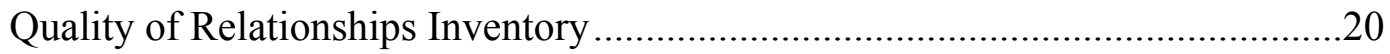

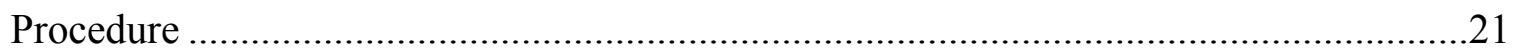

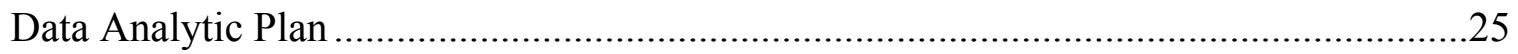

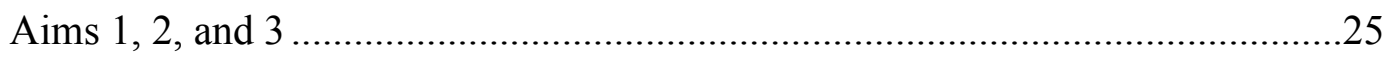

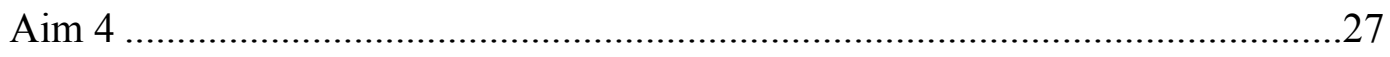

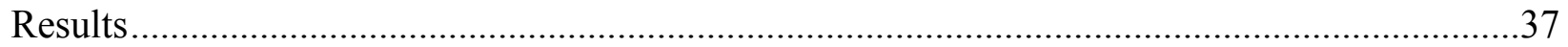

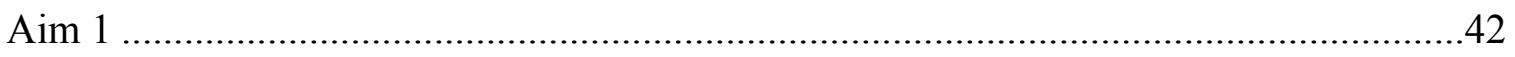

Aim 2

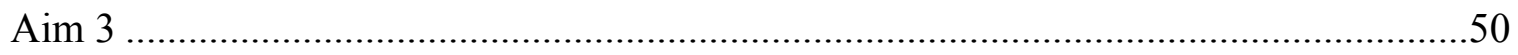

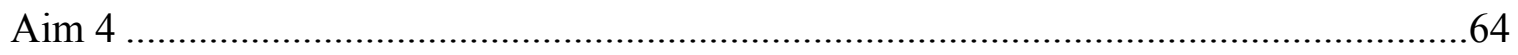

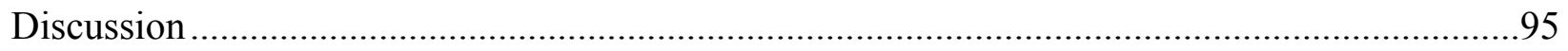

Trajectory of PTSD Following Trauma Exposure: Aim 1...............................................96

Trajectory of Interpersonal Relationship Functioning Following Trauma Exposure: Aims

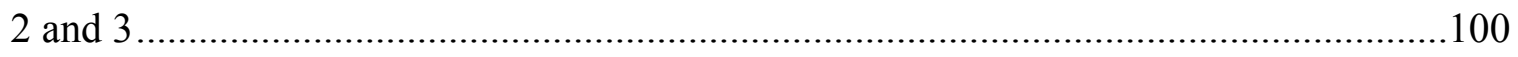

Associations between Initial Levels of Interpersonal Relationship Functioning and

Projected End-state PTSD Symptom Severity: Aims 2 and 3 ......................................104

Directionality of the Association between PTSD and Interpersonal Relationship

Functioning Following Trauma Exposure: Aim 4 ………........................................107 


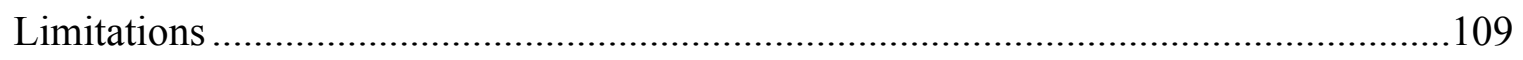

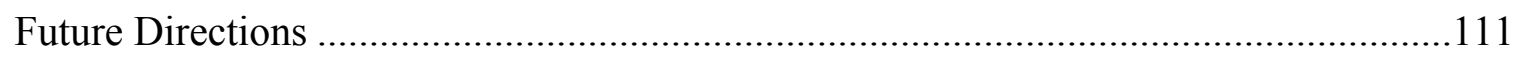

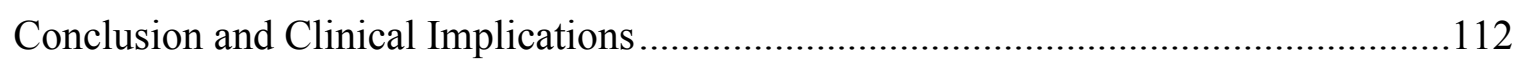

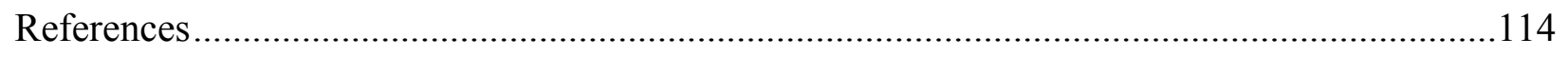




\section{List of Tables}

Table 1: Prevalence of DSM-IV-TR Current and Lifetime Mental Health Conditions among Participants at their Initial Assessment

Table 2: Means and Standard Deviations for Outcome Variables across Assessment Points ....38

Table 3: Correlations between Outcomes Variables, and Means and Standard Deviations for Outcome Variables Separated by Trauma Type and Close Other Type across Assessment Points

Table 4: Growth Curve Models Predicting Initial PTSD Symptom Severity and Symptom Trajectory from Projected End-State PTSD Severity

Table 5: Growth Curve Models Predicting Interpersonal Relationship Functioning Initial Status and Trajectory from Projected End-State PTSD Severity .46

Table 6: Growth Curve Models Predicting Interpersonal Relationship Functioning Initial Status and Trajectory by Projected End-State PTSD Severity, Trauma Type, and their Interaction

Table 7: Growth Curve Models Predicting Interpersonal Relationship Functioning Initial Status and Trajectory by Projected End-State PTSD Severity, Type of Close Other, and their Interaction.

Table 8: Descriptive Statistic for Time Structures .65

Table 9: Parameter Estimates and Deviance Statistics from Growth Curve Models used to Evaluate Time Structures .66

Table 10: Model Fit Estimates and Results of the Chi-Square Difference Tests for the Univariate Latent Difference Score Models. .68 
Table 11: Model Parameter Estimates for the Best Fitting PTSD Univariate Latent Difference Score Models.

Table 12: Model Parameter Estimates for the Best Fitting Interpersonal Relationship Functioning Univariate Latent Difference Score Models . .73

Table 13: Model Fit indices and Parameter Estimates for the CAPS with QRI-Support Bivariate

Latent Difference Score Model . .79

Table 14: Model Fit indices and Parameter Estimates for the PCL with QRI-Support Bivariate Latent Difference Score Model .82

Table 15: Model Fit indices and Parameter Estimates for the CAPS with QRI-Conflict Bivariate Latent Difference Score Model .85

Table 16: Model Fit indices and Parameter Estimates for the PCL with QRI-Conflict Bivariate Latent Difference Score Model

Table 17: Model Fit indices and Parameter Estimates for the CAPS with QRI-Depth Bivariate Latent Difference Score Model

Table 18: Model Fit indices and Parameter Estimates for the PCL with QRI-Depth Bivariate Latent Difference Score Model 


\section{List of Figures}

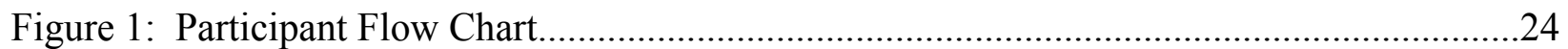

Figure 2: Path Diagrams For The Six Univariate Latent Difference Score (LDS) Models Based on a 5 Segment Time Structure

Figure 3: Trajectories of PTSD Symptoms Over Time According to the CAPS and PCL across High and Low Levels of Projected End-state PTSD .44

Figure 4: Trajectories of the QRI Subscales across High and Low Levels of Projected End-state

$$
\text { PTSD }
$$

Figure 5: Trajectories of the QRI Subscales by Type of Trauma Exposure across High and Low

Levels of Projected End-state PTSD

Figure 6: Trajectories of the QRI Subscales by Type of Close Other across High and Low

Levels of Projected End-state PTSD

Figure 7: Path Diagram for the Bivariate Latent Difference Score (LDS) Model for Dual Variable Proportional Change for PTSD and Constant Change for Interpersonal

Relationship Functioning

Figure 8: Path Diagram for the Bivariate Latent Difference Score (LDS) Model for Dual Variable Proportional Change for PTSD and Fixed Proportional Change for Interpersonal Relationship Functioning .77 


\section{Posttraumatic Symptomatology, Interpersonal Relationship Functioning, and their Association Following Trauma Exposure}

Exposure to a traumatic event results in a diagnosis of posttraumatic stress disorder (PTSD) among 7\% to 9\% of the North American population during their lifetime (Breslau et al., 1998; Kessler, Sonnega, Bromet, Hughes, \& Nelson, 1995; Van Ameringen, Mancini, Patterson, \& Boyle, 2008). Across individuals diagnosed with this condition, difficulties in interpersonal relationship functioning (e.g., intimate relationship, family, and social functioning) have been well documented (for reviews, see Galovski \& Lyons, 2004; Lambert, Engh, Hasbun, \& Holzer, 2012; Monson, Taft, \& Fredman, 2009; Taft, Watkins, Stafford, Street, \& Monson, 2011). Although the trajectory of PTSD symptom expression following traumatization is reasonably well understood (i.e., most individuals exhibit PTSD symptoms immediately following exposure that remit over time; Riggs, Rothbaum, \& Foa, 1995; Rothbaum, Foa, Riggs, Murdock, \& Walsh, 1992), there is minimal research examining the trajectory of interpersonal relationship functioning following trauma exposure. In fact, many of the studies in this area have only examined interpersonal difficulties among individuals with longstanding PTSD and in a crosssectional nature (e.g., Allen, Rhoades, Stanley, \& Markman, 2010; King, Taft, King, Hammond, \& Stone, 2006; Laffaye, Cavella, Drescher, \& Rosen, 2008; Melvin, Gross, Hayat, Jennings, \& Campbell, 2012; Solomon, Debby-Aharon, Zerach, \& Horesh, 2011), paying little or no attention to how interpersonal functioning may change over time posttrauma. In this vein, difficulties in interpersonal relationship functioning have typically been framed as a consequence of the disorder. However, the few longitudinal studies that that have examined social support, an aspect of interpersonal relationship functioning, suggest the association between social support and PTSD seems to change over time (e.g., Kaniasty \& Norris, 2008). 
Although problematic interpersonal relationship functioning has traditionally been framed as a consequence of PTSD, the direction of the association between PTSD and interpersonal functioning may differ as a function of time since trauma exposure. Accordingly, understanding the nuances of the associations between these variables over time posttrauma may be critical to identifying viable intervention targets to promote posttraumatic recovery and prevent chronic PTSD. Moreover, in addition to social support, relationship researchers have identified various dimensions of interpersonal relationship functioning such as cohesion, conflict, depth, and expressiveness (e.g., Berscheid, Snyder, \& Omoto, 1989; Moos \& Moos, 1981; Pierce, Sarason, Sarason, Solky-Butzel, \& Nagle, 1997). To date, no studies have examined interpersonal relationship functioning more broadly or taken into consideration that these other dimensions may be differentially associated with PTSD. It is important to consider these broader dimensions of functioning because they may present unique opportunities to influence one's PTSD symptom trajectory posttrauma. Thus, the purpose of the current study was to examine the trajectory of posttraumatic symptomatology and interpersonal relationship functioning, following recent trauma exposure and to examine the effects that these constructs exert on one another over time.

\section{Trauma Exposure and Posttraumatic Stress Disorder}

The Diagnostic and Statistical Manual of Mental Disorders, Fourth Edition, Text Revision (DSM-IV-TR; American Psychiatric Association, 2000) defines trauma as an event that "involved actual or threatened death or serious injury, or a threat to the physical integrity of self or others." (p. 467). These events are varied, and include such experiences as physical and sexual assault, military combat, kidnapping, abduction, torture, motor vehicle accidents, childhood sexual or physical abuse, and natural or manmade disasters. Moreover, the DSM-IV-TR 
indicates that exposure to these events can take the form of directly experiencing, witnessing, or being confronted with them, as well as learning about an event occurring to a close other (i.e., family member or close friend).

PTSD is a condition that can result following trauma exposure. As per the DSM-IV-TR, PTSD is considered to be present if a trauma occurred during which one's response included intense fear, helplessness, or horror, and is followed by the presence of a number of psychological symptoms lasting for at least a month-long period which cause significant distress or functional impairment. These symptoms are classified into three clusters, which include reexperiencing symptoms (e.g., intrusive memories, distressing dreams, flashbacks), avoidance and emotional numbing symptoms (e.g., efforts to avoid internal and external reminders of the trauma, lack of interest, difficulty feeling emotions), and arousal symptoms (e.g., sleep difficulties, irritability and anger, hypervigilance; American Psychiatric Association, 2000).

Epidemiological research suggests that $55 \%$ to $90 \%$ of North Americans will experience at least one traumatic event at some point in their lifetime (Breslau et al., 1998; Kessler et al., 1995; Van Ameringen et al., 2008). Although PTSD can result from exposure to any one of these events, traumas that include an element of interpersonal violence (e.g., rape, sexual/physical assault, combat) appear to present an increased risk for PTSD (Breslau et al., 1998; Frans, Rimmö, Åberg, \& Fredrikson, 2005; Kessler et al., 1995). Conceptually, these events can be thought of as occurring as a result of one's actions against another. This conceptualization has been corroborated by a recent study examining trauma type as a predictor of PTSD in a large sample of Canadian Forces members, which found that interpersonal and military-related traumas were more strongly associated with a PTSD diagnosis compared with a number of other traumatic events (Wanklyn et al., submitted). 
Research has shown that most individuals will experience PTSD-like symptoms in the days and weeks following traumatization (Blanchard, Hickling et al., 1996; Riggs et al., 1995; Rothbaum et al., 1992). For the majority of people, symptoms will remit with time (i.e., natural recovery), with substantial symptom reduction within the first 3 months post-exposure. However, the trajectory of symptom reduction has been shown to extend into the 6- to 12-month period post-exposure among many individuals (Blanchard, Hickling et al., 1996). Although most recover, a significant minority will go on to receive a diagnosis of PTSD (Breslau et al., 1998; Kessler et al., 1995; Van Ameringen et al., 2008). Given this pattern of symptom manifestation and remittance, PTSD is best conceptualized as a disorder of impeded recovery, whereby factors are believed to interfere with one's natural recovery.

\section{Interpersonal Relationship Functioning and Posttraumatic Stress Disorder}

Meta-analytic studies examining correlates of PTSD have identified a number of variables that influence one's risk for the disorder (Brewin, Andrews, \& Valentine, 2000; Ozer, Best, Lipsey, \& Weiss, 2003). Brewin and colleagues investigated 14 risk factors across 77 studies. Their results suggest that peri- and posttraumatic variables have larger effects compared with pre-trauma factors. Specifically, they found that trauma severity, posttrauma life stress, and posttrauma social support were the strongest predictors of PTSD. Ozer and colleagues' metaanalysis excluded an examination of demographic factors (e.g., gender, socioeconomic status); instead, they focused on psychological factors related to trauma. They examined seven predictors across 68 studies and found perceived life threat at the time of traumatization, perceived social support posttrauma, peritraumatic dissociation, and peritraumatic emotions (e.g., fear, helplessness, horror, guilt, shame) to be the strongest predictors of PTSD. Similar to Brewin et al., Ozer and colleagues also concluded that peritraumatic and posttraumatic variables were the 
strongest predictors of the disorder.

Although informative, a key problem with many of the identified risk factors is that they are difficult or impossible to target because they occur during the trauma and cannot be controlled or anticipated (e.g., peritraumatic dissociation, trauma severity, perceived life threat). As a result, they are ineffective targets in the prevention of PTSD. In contrast, one important variable that is dynamic in nature and identified in both meta-analyses to be among the strongest and most robustly associated risk/resilience factors for PTSD is posttraumatic social support (Brewin et al., 2000; Ozer et al., 2003). More specifically, Brewin et al. (2000) found the weighted average correlation between a lack of posttrauma social support and PTSD to be 40 . Likewise, Ozer and colleagues (2003) found the weighted average association between perceived support posttrauma and PTSD to be -.28. Social support, and other aspects of interpersonal relationship functioning, present a unique opportunity as a risk/resilience factor, because they are variables that can more easily be targeted in the wake of traumatization.

The association between PTSD and interpersonal relationship functioning problems has been well documented across various samples differing in sex, trauma type, nationality, and military/veteran status (for reviews, see Galovski \& Lyons, 2004; Lambert et al., 2012; Monson et al., 2009; Renshaw, Blais, \& Caska, 2011; Taft et al., 2011). In their review of PTSD and intimate relationships, Monson and colleagues (2009) propose three lenses by which to evaluate the association between PTSD and interpersonal relationship functioning, with differing levels of theoretical attention and empirical support accumulated for each. The first frames relationship dysfunction as an outcome of PTSD, the second frames relationship functioning as a risk/resilience factor for PTSD, and the third frames the association between PTSD and relationship functioning as a bidirectional process. Below I briefly review the empirical and 
theoretical literature for the three frameworks offered by Monson and colleagues, with the additional consideration of time since trauma exposure.

\section{Interpersonal relationship dysfunction as an outcome of posttraumatic stress}

disorder. Interpersonal relationship problems have typically been framed as consequence of PTSD (e.g., Allen et al., 2010; Erbes, Meis, Polusny, \& Compton, 2011; Erbes, Meis, Polusny, Compton, \& Wadsworth, 2012; Melvin et al., 2012; Solomon et al., 2011). However, many of the studies that support the association in this direction have been cross-sectional in nature (e.g., Allen et al., 2010; Melvin et al., 2012; Solomon et al., 2011), limiting the utility of the findings in establishing a causal pathway. Recent longitudinal studies with US soldiers and veterans provide support for the notion that PTSD has deteriorating effects on interpersonal relationships. For example, among US male National Guard soldiers, PTSD symptom severity within the first year post-deployment was predictive of their female partners' relationship adjustment 6 to 9 months later, after controlling for baseline relationship adjustment (Erbes et al., 2012). When tested in the opposite direction of causality, the association was not statistically significant (i.e., relationship adjustment was not predictive of PTSD over time).

Several studies have examined social support more generally, rather than exclusively from intimate relationships, and its association with PTSD symptomatology in US veterans. King, Taft et al., (2006) found a negative association between PTSD symptoms 2 years postdeployment and social support 7 years later, among male US Gulf War veterans; the opposite direction of association was not statistically significant (i.e., social support was not predictive of PTSD over time). Similarly, Laffaye and colleagues' (2008) studied a group of mostly US Vietnam era veterans with a longstanding history of PTSD, who were assessed 6 to 24 months after they had completed a residential PTSD treatment program and then again 6 months later. 
Perceived interpersonal resources and stressors from four different sources (i.e., spouse, relatives, non-veteran friends, and veteran peers) and PTSD symptom severity were assessed. PTSD symptom severity at the first assessment was associated with erosion of interpersonal resources from non-veteran friends at the 6-month follow up assessment. A similar, although non-significant, trend was found for interpersonal resources from veteran peers, whereas no association was detected for interpersonal resources from relatives or interpersonal stressors from any of the sources. Unfortunately, these associations could not be tested in reference to spouses due to sample size restrictions. Additionally, when tested in the opposite direction, neither interpersonal resources nor stressors from any of the sources at the first assessment were predictive of PTSD symptom severity at the follow-up assessment.

In summary, these findings provide evidence for the influence of PTSD on interpersonal relationship functioning. Although the extant longitudinal studies have not placed an explicit emphasis on time since trauma exposure, two of these three studies have been with individuals with long-standing PTSD (King, Taft et al., 2006; Laffaye et al., 2008). Although the third examined individuals more proximally to exposure (Erbes et al., 2012), there was a large window of recruitment (i.e., within 1 year following a 16-month deployment), which potentially resulted in a significant time lag between exposure and assessment. Taken together, these studies support the deteriorating effect of PTSD on interpersonal relationship functioning, but this association may only occur among individuals who have longer-standing PTSD.

\section{Interpersonal relationship functioning as a risk/resilience factor for posttraumatic}

stress disorder. In contrast to that outlined above, some have viewed interpersonal relationship functioning as a risk or resilience factor influencing the recovery trajectory following trauma exposure (e.g., Perry, Difede, Musngi, Frances, \& Jacobsberg, 1992; Skopp et al., 2011). In this 
framework, interpersonal relationship problems are viewed as a risk factor that contributes to and maintains PTSD symptomatology, whereas adaptive relationship functioning may promote posttraumatic recovery.

In a longitudinal study of hospitalized burn victims, Perry and colleagues (1992) examined objective (e.g., percent of burned area) and subjective (e.g., perceived social support, emotional distress) variables within the first week of injury. They found that perceived emotional support negatively and significantly predicted a diagnosis of PTSD at assessments conducted 2, 6 , and 12 months posttrauma. Notably, none of the other variables measured at 1-week postinjury were predictive of PTSD at the 6- and 12-month follow-up assessments (i.e., percentage of body surface area burned, general distress, intrusive and avoidant thoughts, facial disfigurement).

Evidence for this perspective has more recently emerged from a large longitudinal study of male and female US military service members who were assessed 45 to 120 days prior to deployment and 90 to 180 days post-deployment (Skopp et al., 2011). Examining a subgroup of the sample that endorsed having a spouse or significant other at both pre- and post-deployment assessments, they found that $40 \%$ of soldiers reported reductions in their relationship strength (i.e., responses to a single item on a 5-point scale from the Quality of Marriage Index; Norton, 1983). Using an index of pre- to post-deployment change in relationship strength, they examined whether change in relationship strength interacted with combat exposure and sex to predict the odds of screening positive for PTSD post-deployment. Overall they found a positive association between level of combat exposure and likely PTSD. However, among female soldiers, changes in the strength of their intimate relationship was negatively associated with risk for likely PTSD, but only when they had been exposed to higher, but not lower, levels of combat. In other words, women who had been exposed to more combat and who had reductions in their relationship 
satisfaction were at greater risk for PTSD. In contrast, the association between combat exposure and likely PTSD did not change as a function of changes in relationship strength for men (Skopp et al., 2011).

\section{The association between interpersonal relationship functioning and posttraumatic} stress disorder as bidirectional and time-dependent. Two theoretical models have been put forth that posit reciprocal associations between interpersonal relationship functioning and PTSD (Monson, Fredman, \& Dekel, 2010; Nelson Goff \& Smith, 2005). Although initially developed in the context of intimate relationship functioning, one of these models has also been elaborated to include other, non-intimate, dyads (Monson et al., 2010).

The couple adaptation to traumatic stress (CATS) model (Nelson Goff \& Smith, 2005) provides a framework for how couples respond following trauma. Specifically, it proposes that there are three factors that interact to influence adaptation: each partner's individual functioning, predisposing factors and resources, and couple-level functioning. Individual- and couple-level functioning are considered to be determined and influenced by the predisposing factors and resources. The model suggests that a trauma-exposed individual will initiate a response to trauma that can interact bidirectionally with the other factors in the model to ultimately influence each partner's trauma-related symptoms.

Cognitive-behavioural interpersonal theory (C-BIT) of PTSD (Monson et al., 2010) holds that individual- and dyad-level factors interact with one another to promote trauma recovery or maintain PTSD symptomatology in the wake of trauma exposure. Specifically, it is proposed that trauma-exposed individuals, and their significant others, each have cognitive, behavioural, and emotional factors that interact within each individual and between partners to influence the relationship milieu. In turn, relationship factors feed back on individual functioning. Elements of 
each factor may be pre-existing or may develop in the posttrauma period.

In this model, a trauma-exposed individual's pre-existing beliefs and schemas (i.e., just world belief) can work to confirm maladaptive cognitions about why the trauma occurred. This can then translate into maladaptive cognitions about his/her ability to navigate through the world effectively and manage other stressful events in the future. Moreover, the trauma-exposed individual may refuse to engage in tasks that remind him/her of the trauma or that causes him/her distressing emotions. As a result, his/her close significant other may begin taking on additional duties within the relationship and may develop his/her own beliefs about the trauma-exposed individuals' ability to cope or tolerate distress. This may lead to additional tension and stress on the relationship by virtue of the close significant other feeling burdened or overwhelmed by the added duties that he/she now needs to take on. Moreover, although a significant other's behaviours, and corresponding beliefs, may be designed to prevent a trauma-exposed partner from experiencing distress, they may actually function to maintain PTSD by preventing new learning and promoting avoidance behaviour. Over time, each partners' cognitions, behaviours, and emotions, and their associations, begin affecting the dyad's relationship functioning. This, in turn, can work to facilitate the development of additional maladaptive or distress-maintaining beliefs about each partner, PTSD, and the relationship overall.

Both the CATS model and C-BIT of PTSD contend that the associations between significant others' responses in the posttraumatization period are critical to understanding a dyad's overall adjustment to trauma and PTSD. However, both models neglect to discuss the potential developmental nature of the association. In other words, time since trauma exposure seems to influence the direction of the pathway between PTSD symptomatology and relationship functioning. In light of the empirical literature reviewed earlier, the association between 
interpersonal relationship functioning and PTSD may not be fully bidirectional across the recovery or posttraumatization period. Rather, it may change direction as a function of time since traumatization, such that interpersonal relationship functioning may be protective against PTSD or a risk factor for it in the more proximal aftermath of trauma exposure, whereas PTSD may have a deteriorating effect on interpersonal relationship functioning in the more distal aftermath of exposure.

To date, only one empirical study has documented the time dependent nature of the associations between interpersonal variables and PTSD. Kaniasty and Norris (2008) investigated PTSD and perceived social support from one's family in a large sample of individuals who had survived significant flooding and mudslides in Mexico. Individuals were assessed 6, 12, 18, and 24 months following the disaster. Using a cross-lagged panel design, they investigated the associations between PTSD symptomatology and perceived social support across time. They found that social support assessed 6 months following the disaster was predictive of PTSD symptom severity at the 12-month assessment. In contrast, PTSD symptom severity assessed 18 months post-exposure was found to significantly deteriorate social support after 24 months. PTSD symptoms and social support assessed at 12 months post-exposure were mutually predictive of one another at the18-month assessment. They concluded that social support early on after trauma exposure may determine the presence of PTSD, but as it becomes more chronic, PTSD begins to erode social support.

These findings suggest that time since trauma exposure may be a critical factor that influences the direction of the association between PTSD and interpersonal relationship functioning. However, only one study to date has investigated this varying directionality hypothesis and it was limited to familial social support within flood/mudslide victims. The 
literature reviewed above suggests that the first year following exposure may be a critical period during which interpersonal variables are predictive of PTSD (e.g., Perry et al., 1992; Skopp et al., 2011), whereas as time since trauma exposure increases, PTSD seems to predict interpersonal relationship functioning. Although some research suggests that the deteriorating effect of PTSD on relationship functioning may not take effect until beyond 1-year posttrauma (e.g., Kaniasty \& Norris, 2008; King, Taft et al., 2006), an explicit timeline requires further empirical investigation and validation, primarily because the research establishing this deteriorating effect has been conducted mostly among individuals with longstanding PTSD (e.g., King, Taft et al., 2006; Laffaye et al., 2008).

\section{Current Study}

The current study sought to investigate posttraumatic symptomatology and interpersonal relationship functioning over time, following trauma exposure. These constructs were examined within a community sample of individuals exposed to diverse traumatic events within the 6 months prior to enrolling into the study, and who were followed for 1 year from their date of enrollment. Based on the existing literature, four specific aims and corresponding hypotheses were put forward.

Aim 1. To investigate the existing conceptualization of natural recovery following trauma exposure, the first aim was to examine the trajectory of PTSD symptom severity over 1.5 years following trauma exposure. Specific to Aim 1, it was hypothesized that on average, PTSD symptom severity would decline over time. Additionally, it was hypothesized that the course of PTSD symptom severity following trauma exposure would differ by end-state PTSD (i.e., PTSD diagnostic status at the final assessment), such that individuals without end-state PTSD would evidence faster reductions in their symptomatology over time compared with those with end- 
state PTSD.

Aim 2. The second aim of the current study was to examine the trajectory of interpersonal relationship functioning over time, following trauma exposure. Given the limited knowledge of the trajectory of relationship functioning following trauma exposure, no specific hypotheses were put forth. However, the existing research on interpersonal functioning and PTSD suggests a negative association between these variables over time; thus, it was anticipated that the course of relationship functioning following trauma exposure would differ by end-state PTSD, such that individuals with end-state PTSD would evidence greater worsening of their relationship functioning over time, compared with those without end-state PTSD.

Aim 3. The third aim of this study was to examine two potential moderators of the course of interpersonal relationship functioning after trauma exposure among those with end-state PTSD. These two moderators were type of trauma (i.e., interpersonal vs. non-interpersonal) and type of close other relationship (i.e., intimate vs. non-intimate relationship). Despite a relative absence of research examining the effects that these variables have on the course of interpersonal relationship functioning over time, speculative hypotheses were offered. Specifically, it was hypothesized that among individuals with end-state PTSD, those exposed to interpersonal traumas would evidence greater worsening of their interpersonal relationship functioning over time, compared with those exposed to non-interpersonal traumas. Similarly, it was hypothesized that among individuals with end-state PTSD, those documenting their relationship functioning in reference to an intimate partner would evidence greater reductions in interpersonal relationship functioning over time, compared with those documenting their relationship functioning in reference to a non-intimate relationship.

Aim 4. As outlined above, the direction of the associations between PTSD and 
interpersonal relationship functioning may be dependent on time since traumatization. Therefore, the fourth aim of the current study was to investigate associations between PTSD symptom severity and interpersonal relationship functioning over time, following trauma exposure. It was hypothesized that the direction of the negative associations between PTSD symptom severity and interpersonal relationship functioning would differ over time.

Given that participants were able to enroll into the study within 6 months of experiencing a trauma and were then followed for 1 year from their date of enrollment, each participant had a different assessment schedule in relation to the amount of time that had passed since their trauma exposure. Additionally, the range of enrollment dates allowed for the earliest initial assessment to occur as quickly as 1 day post-exposure (for those who enrolled immediately) and latest final assessment to occur a maximum of 18-months post-exposure (for those who enrolled 6 months post-exposure). It was planned for the entire sample to provide assessments that fell within this time period ( 1 day to 18 months post exposure) across their various assessment points. Accordingly, it was hypothesized that within the first year of exposure, interpersonal relationship functioning would significantly predict subsequent changes in PTSD symptom severity, whereas when assessed beyond one year post-exposure, PTSD symptom severity would significantly predict subsequent changes in interpersonal relationship functioning.

\section{Method}

\section{Participants}

The current study was derived from a larger, ongoing longitudinal study assessing trauma-exposed individuals and their close significant others following trauma exposure. Relevant to the current study, 107 dyads were recruited from the community. Each dyad consisted of an individual who had been exposed to a traumatic event within 6 months of their 
date of enrollment into the study, and a close significant other (e.g., intimate partner, family member, close friend) who was aware that the event occurred but was not exposed to it. The sample consisted of $31(29 \%)$ men and 76 (71\%) women. At the time of their first assessment, participants' mean age was $35.51(S D=13.49)$ years. The majority of participants $(56.1 \%)$ were single (never married), $16.8 \%$ were in a committed relationship, but not married, $12.1 \%$ were married, 14\% were separated, widowed, or divorced. Nearly half $(48.6 \%)$ of the participants were white/Caucasian, 15.9\% were black (e.g., African, Haitian, Jamaican, Somali), 3.7\% were Aboriginal, 13.1\% were South Asia, East Asian or South East Asian, 3.7\%, were Arab or West Asian, 4.7\%, were Latin American, and 10.3\% self-identified as having a mixed, or being of another, ethnicity. Participants' average yearly income ranged from under $\$ 5,000$, to over $\$ 100,000$, and over half (62.6\%) were either currently employed or enrolled in an education program. Not counting a potential diagnosis of PTSD associated with the traumatic event that led to enrollment into the current study, $59.8 \%$ of participants met criteria for a current mental health condition, and $64.5 \%$ had a lifetime history of a mental health condition as per the DSM-IV-TR. Table 1 presents descriptive statistics with regard to participants' current and lifetime mental health conditions. Notably, approximately a third (34.6\%) of participants were utilizing some kind of mental healthcare (e.g., psychotropic medication, psychotherapy) at the time of the their initial assessment. 
Table 1

Prevalence of DSM-IV-TR Current and Lifetime Mental Health Conditions among Participants at their Initial Assessment.

\begin{tabular}{lcc}
\hline & Current & Lifetime \\
& $n(\%)$ & $n(\%)$ \\
Mood Disorder & $43(40.2 \%)$ & $57(53.3 \%)$ \\
Anxiety Disorder (excluding PTSD) & $26(24.3 \%)$ & $30(28.0 \%)$ \\
PTSD (related to another event) & $15(14.0 \%)$ & $19(17.8 \%)$ \\
Substance Use Disorder & $26(24.3 \%)$ & \\
Psychotic Disorder & $2(1.9 \%)$ & $3(2.8 \%)$ \\
Eating Disorder & $5(4.7 \%)$ & \\
\hline
\end{tabular}

Note. The prevalence figures for PTSD reflect PTSD as result of a

traumatic event other than the event that led to participation in the current study. 
With regard to the traumatic events that led to enrollment into the current study, $30.8 \%$ of the participants were exposed to an accident (e.g., motor vehicle accident, accidental burn), $25.2 \%$ were exposed to a physical assault, $21.5 \%$ were exposed to a sexual assault, $9.3 \%$ were exposed to sudden illness or death, $3.7 \%$ were exposed to a robbery or a home invasion, $1.9 \%$ were exposed to threats, $.9 \%$ were held hostage, and $6.5 \%$ reported exposure to a different traumatic event. Notably, none of the participants in the sample were exposed to combat-related trauma. Participants were also categorized with regard to the type of exposure to their traumatic events (i.e., directly experiencing, witnessing, or learning about the trauma). The majority $(81.3 \%)$ of participants in the sample directly experienced their traumatic event, $15.9 \%$ witnessed it occur, and .9\% learned about it happening. Additionally, based on criteria established from findings from previous empirical studies (Breslau et al., 1998; Frans et al., 2005; Kessler et al., 1995) participants were classified with regard to whether they had been exposed to interpersonal trauma, defined as having directly experienced a physical or sexual assault, threats, or being the victim of a hostage situation, robbery, or home invasion. Just over half $(51.4 \%)$ of participants were classified as having experienced interpersonal traumatic events. Although participants had to enroll into the study within 6 months of exposure to their traumatic events, due to scheduling and availability of assessors and participants, some participants received their initial assessment beyond 6 months following trauma exposure. Accordingly, the length of time between trauma exposure and participants' initial assessments ranged from 24 to $260(M=124.22, S D=52.78)$ days following trauma exposure.

With regard to the types of relationship between participants and the close significant others enrolled in the study with them, just under half (46.7\%) of participants enrolled in the study with a friend, $22.4 \%$ enrolled with an intimate partner, $14 \%$ enrolled with a parent or 
parental figure, 9.3\% enrolled with a sibling, 3.7\% enrolled with a child, and 3.7\% enrolled with an extended family member. Less than half of the sample (37.4\%) reported living with the close significant other with whom they enrolled into the study.

\section{Measures}

Clinician-administered interviews were administered to trauma-exposed participants by clinical psychology graduate students trained in the administration of these instruments. Additionally, trauma-exposed participants completed self-report measures, either in hard copy or electronically using an online survey administration program (Qualtrics, 2005). Close significant others did not complete measures for the purpose of this study, but their relationships with the trauma-exposed individuals served as the basis for responding for measures in which the traumaexposed participant was asked about interpersonal relationship functioning.

Clinician-Administered PTSD Scale. The Clinician-Administered PTSD Scale (CAPS; Blake et al., 1995) is a semi-structured clinical interview that assesses for DSM-IV-TR PTSD diagnostic criteria and symptom severity. After assessing for the presence of a DSM-IV-TR Criterion A traumatic event, each of the 17 DSM-IV-TR PTSD symptoms are assessed with regard to their frequency and intensity over the past month. Both frequency and intensity are rated on a 5-point scale (0-4). Consistent with previous research (e.g., Weathers, Keane, \& Davidson, 2001), items endorsed with a frequency equal to or greater than one, and an intensity of equal to or greater than two are considered to meet the minimum threshold to count as a symptom of PTSD. In addition to meeting the necessary symptom threshold (i.e., one reexperiencing symptom, three avoidance/emotional numbing symptoms, and two hyperarousal symptoms), an overall severity rating (i.e., sum of the frequency and intensity ratings on all items) equal to or greater than 45 is necessary for a PTSD diagnosis. Psychometric studies 
support the reliability and validity of this measure, with strong inter-rater reliability on the global severity score (.89), total frequency score (.92-1.00) and total intensity score (.93-.98; Hovens et al., 1994). Additionally, test-retest reliability and internal consistency across the DSM-IV-TR PTSD symptoms clusters are also strong when considered individually $(r=.77-.96 ; \alpha=.85-.87)$ and altogether $(r=.90-.98 ; \alpha=.94$; Blake et al., 1995). The CAPS has also been shown to have high convergent validity with the PTSD module of the Structured Clinical Interview for DSM-IV (SCID; Foa \& Tolin, 2000).

In the current study, the CAPS was used as the primary measure of PTSD diagnostic status and symptom severity. Additionally, participants' descriptions of their traumas provided during the administration of the CAPS were used to determine the type of trauma and type of exposure. To assess the reliability of the administration of the CAPS, a subset (8.5\%) of the interviews conducted were randomly selected for review by an independent assessment monitor, resulting in an excellent intra-class correlation coefficient (ICC) between the assessors' ratings and the independent assessment monitor's rating $(\mathrm{ICC}=.99)$. Additionally, the kappa coefficient with regard to PTSD diagnostic status was good between the assessors' ratings and the independent assessment monitor's rating $(\kappa=.87)$. Internal consistency for the CAPS was excellent across all assessment point $(\alpha \mathrm{s}=.94$ to .96$)$.

\section{MINI International Neuropsychiatric Interview. The MINI International} Neuropsychiatric Interview (MINI; Lecrubier et al., 1997) is a semi-structured clinical interview that assesses for the presence of past and current DSM-IV-TR Axis I disorders. It has been shown to have good concordance with the other clinician administered interviews for DSM Axis I disorders (Sheehan et al., 1998). In the current study, the MINI was used to characterize and describe the sample. 
Mental Health Care Utilization. The Mental Health Care Utilization (MHU) measure is a clinician-administered interview designed to assess for utilization of psychosocial and psychopharmacological treatments. Individuals are asked about the type, frequency, and provider of treatment. Currently, there is no available psychometric research on the MHU; however, this measure has been used in previous PTSD studies (e.g., Monson et al., 2012; Schnurr et al., 2007). In the current study, the MHU was used to characterize and describe the sample.

PTSD Checklist. The trauma specific version of the PTSD Checklist (PCL; Weathers, Litz, Herman, Huska, \& Keane, 1993) is a self-report measure that assesses the severity of PTSD symptoms, from a specific traumatic event, consistent with the DSM-IV-TR over the past month. The PCL consists of 17 items rated on a 5-point scale (1-5). Research supports the psychometric properties of the PCL with a total score correlation between the PCL and the CAPS of $r=.93$ and diagnostic efficiency of .90 when using a diagnostic cut-off of 44 (Blanchard, JonesAlexander, Buckley, \& Forneris, 1996). In the current study, the PCL was used as a secondary measure of PTSD symptom severity. Internal consistency for the PCL was excellent across all assessment point ( $\alpha$ s $=.94$ to .96$)$.

Quality of Relationships Inventory. The Quality of Relationships Inventory (QRI; Pierce, Sarason, \& Sarason, 1991) is a self-report measure that assesses the quality of one's relationship to a close significant other (e.g., intimate partner, family member, close friend). The QRI contains 25 items rated on a 4-point scale (1-4) and has three subscales assessing support, conflict, and depth of the relationship being assessed. The support subscale (QRI-Support) assesses the perceived availability of social support from the close other in reference. The conflict subscale (QRI-Conflict) assesses the degree to which the relationship in reference is a source of conflict. The depth subscale (QRI-Depth) assesses the degree to which the relationship 
in reference is perceived to be positive, important, and secure. Previous research demonstrates that the QRI subscales have adequate internal consistency, as well as good concordance when relationships are assessed by both members of a dyad (Pierce et al., 1997). In the current study, trauma-exposed individuals responded to the items on the QRI in reference to the close other who enrolled into the study with them. Internal consistency for the QRI subscales was strong across all assessment points (QRI-Support $\alpha \mathrm{s}=.85$ to .93; QRI-Conflict $\alpha \mathrm{s}=.92$ to .93; QRI-

Depth $\alpha$ s $=.88$ to .92$)$.

\section{Procedure}

The current study was part of a larger ongoing study examining trauma-exposed individuals and their close significant others following trauma exposure. Though the current study documented and reported on the type of relationship between the members of each dyad (i.e., intimate, relative, friend), it focused exclusively on the trauma-exposed partners. Dyads were recruited using newspaper advertisements and flyers posted in the community (universities, hospitals, community centres, local newspapers) and online (facebook, lab website). To increase the external validity of the current study, there were limited inclusion/exclusion criteria. To be eligible to participate, dyads needed to consist of an individual who had been exposed to a DSMIV-TR Criterion A traumatic event within the past 6 months and a close significant other (intimate partner, family member, close friend) who was aware of the event but was not exposed to it. Participation from both the trauma-exposed individual and the close significant other were required in order for the dyad to enroll into the study, and all participants needed to be between the ages of 18 and 75 years. Additionally, all participants had to consent to having their assessments audiorecorded for the purpose of conducting reliability assessments on the clinical interviews. 
Individuals contacted study personnel over telephone or email and were provided with information regarding the purpose of the study and details of participation. Additionally, they received a preliminary screen via telephone to assess inclusion criteria (i.e., age, dyadic nature of participation, audiorecording, trauma exposure). Those who screened positive for inclusion criteria were asked to provide oral consent for participation and to have their study partner (i.e., either the trauma-exposed individual or close significant other) contact study personnel so that they could be provided with study information and details regarding participation. The phone screen was repeated on the second member of the dyad in order to confirm study inclusion criteria. If eligible, this partner was asked to provide oral consent for participation. Dyads who met the inclusion criteria for participation were informed that they could participate in person (i.e., complete the clinical interviews and self-report measures at Ryerson University) or remotely (i.e., complete the clinical interviews over the phone and self-report measures online). They were then asked to review and sign the informed consent form either in person or using an online survey program (Qualtrics, 2005).

Given that the current study focused exclusively on the trauma-exposed partners, the remainder of the procedure is specific to the participation of the trauma-exposed individuals. Trauma-exposed individuals were scheduled for their initial assessment, either remotely or in person, which included a clinician assessment consisting of the CAPS, MINI, and MHU, as well as the administration of self-report measures including the PCL and QRI. Following the initial assessment, participants were scheduled to complete three additional assessments, spaced in 4month intervals, during which the same clinical interviews and self-report measures were administered. Individuals were administered the clinical interviews first and were then required to complete the self-report portion of the assessment within 1 week of completing the interviews. 
Figure 1 depicts participant flow through the study. Participants received $\$ 60$ following their completion of the initial assessment, $\$ 70$ following their completion of the second assessment, $\$ 80$ following their completion of the third assessment, and $\$ 90$ following their completion of their fourth assessment, totaling $\$ 300$ as compensation. Individuals who completed the clinical interviews but failed to complete the self-report measures only received half of the reimbursement offered for the given assessment. 


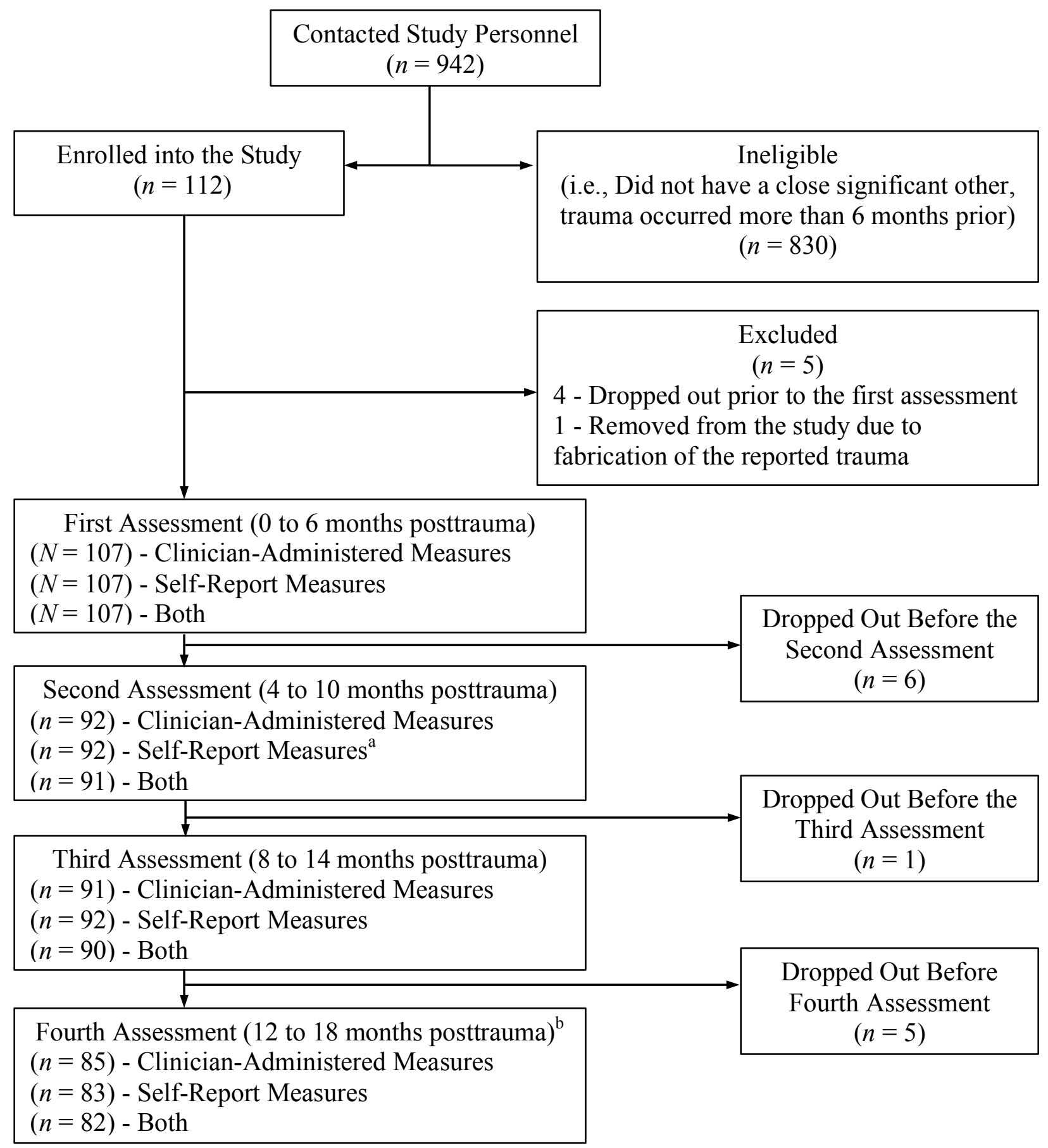

Figure 1. Participant flow chart.

${ }^{a}$ One participant's responses to the PTSD Checklist were deleted due to them receiving the wrong version of the measure.

${ }^{\mathrm{b}}$ The study concluded prior to nine participants having the opportunity to complete their fourth assessment. 


\section{Data Analytic Plan}

Given the longitudinal nature of the data and corresponding research aims, multilevel growth curve modeling and bivariate latent difference score (LDS) modeling, a type of structural equation modeling (SEM), were used to analyze the data.

Aims 1, 2, and 3. Multilevel growth curve modeling was used to examine the trajectory of PTSD symptomatology and interpersonal relationship functioning over time in the posttraumatization period. Multilevel growth curve modeling is a method used to analyze nested data (Raudenbush \& Bryk, 2002; Singer \& Willet, 2003). In the current study, time points were nested within individuals, as each participant was assessed up to four times throughout the study. Multilevel growth curve modeling accounts for the systematic shared variance between data points that occurs via repeated assessment of the same individuals. Accordingly, it compensates for the violation of the assumption of independence of observations that occurs when conducting longitudinal research (Raudenbush \& Bryk, 2002; Singer \& Willet, 2003).

To conduct the analyses relevant to Aims 1, 2, and 3, growth curve models with a 2-level hierarchical structure [time points (Level 1) nested within participant (Level 2)] were evaluated. Models were specified to have random intercepts and slopes (i.e., the intercept and slope of a predictor is permitted to vary across participants) and were estimated using full maximum likelihood estimation. For the purpose of these analyses, time was treated as a continuous variable by calculating the number of days posttrauma at which each assessment occurred, and was centered on the date of trauma exposure. One of the strengths of multilevel growth curve modeling is its flexibility with timing of assessments. It does not require the number or timing of assessments to be invariant across participants.

Consistent with Aim 1, an initial growth curve model was evaluated with time (Level 1) 
predicting PTSD symptom severity (i.e., CAPS and PCL in separate analyses; Level 1), to examine the trajectory of PTSD symptoms following trauma exposure. Next, end-state PTSD diagnostic status, determined at the final assessment, (Level 2) and the time by end-state PTSD diagnostic status interaction (Level 2) were entered into the model, in order to examine differences in initial PTSD symptom severity and symptom trajectory between those who naturally recovered and those who did not. Consistent with Aim 2, an initial growth curve model was constructed with time (Level 1) predicting interpersonal relationship functioning (i.e., QRI; Level 1), in order to examine the trajectory of interpersonal relationship functioning following traumatization. End-state PTSD diagnostic status (Level 2) and the time by end-state PTSD diagnostic status interaction (Level 2) were then entered into the model, in order to examine differences in initial relationship functioning the trajectory of relationship functioning between those who naturally recovered and those who did not.

Consistent with Aim 3, two additional models were assessed to examine the effects of 1) type of trauma and 2) type of close other on interpersonal relationship functioning. First, to examine the influence of type of trauma (interpersonal vs. non-interpersonal) on the trajectory of interpersonal relationship functioning among individuals with end-state PTSD diagnostic status, a model including time (Level 1), end-state PTSD diagnostic status (Level 2$)$, trauma type $(0=$ non-interpersonal trauma, 1 = interpersonal trauma; Level 2), and the interaction of these three terms was used to predict interpersonal relationship functioning (i.e., QRI; Level 1). Second, to examine the influence of type of close other (intimate vs. non-intimate) on the trajectory of interpersonal relationship functioning among individuals with end-state PTSD, a separate model including time (level 1), end-state PTSD diagnostic status (Level 2), close other type $(0=$ nonintimate, 1 = intimate; Level 2), and the interaction of these three terms was used to predict 
interpersonal relationship functioning (i.e., QRI; Level 1). To evaluate both main effects and interactions, these models were constructed hierarchically with main effects entered in the first step and the two- and three-way interactions entered in the second step, as the interpretation of the coefficients associated with the predictor variables differs when an interaction term present (i.e., they represent main effects when no interaction, but represent conditional effects that are influenced by the coding of the other variables with the interaction is present).

Aim 4. To examine the directionality of the associations between PTSD symptomatology and interpersonal relationship functioning over time in the posttraumatization period, bivariate LDS modeling was used (Hamagami \& McArdle, 2001; McArdle, 2001; McArdle \& Hamagami, 2001). LDS models account for autoregressive/proportional change (i.e., change due to the effect of one's level on a given variable on subsequent assessments of the same variable) and nonstationarity/constant change effects (i.e., the mean level change in a variable as a function of time); however, this approach does not allow time to be modeled continuously, and thus does not permit for deviations in the assessment schedule across participants (i.e., the number and timing of assessments must be invariant across participants; see King, King, McArdle, Grimm et al., 2006; King, King, McArdle, Saxe et al., 2006). In the current study, participants enrolled at any point within the first 6 months posttraumatization. Although each subsequent assessment was scheduled at 4-month intervals, depending on the time since trauma exposure at the initial assessment, participants had differing assessment schedules relative to time of traumatization. For example, some participants had their four assessments at 0-, 4-, 8-, and 12-months posttrauma, while others had their four assessments at 6-, 10-, 14-, and 18-months posttrauma. Given that the assessment schedule differed across participants and that LDS modeling cannot accommodate variations in the assessment schedule, growth curve modeling was used to develop 
a time structure for the data.

Fortunately, methods have been developed to allow SEM based LDS modeling to accommodate data structures that do not meet the rigid, invariant data structure restrictions of SEM based LDS models. King and colleagues have described a method to evaluate various time structure specifications in longitudinal trauma research to model time in manner consistent with the SEM based LDS model structure assumptions. Specifically, they advocate for the creation of various time segments (or bins) that can be imposed on data. Various time structure specifications can be compared to one another to identify the time structure that best fits the data using a growth curve modeling framework. By comparing the deviance statistics from the growth curve models that use each imposed time structure, one can identify the best fitting time structure (King, King, McArdle, Grimm et al., 2006). Notably, all participants do not need to have data at each time point. Accordingly, the imposed time structures can differ in their number of time segments.

Using this framework, three time structures were evaluated that utilized four time segments (i.e., the four assessment occasions), five time segments (i.e., Time $1 \leq 120$ days; Time $2=121-240$ days; Time $3=241-360$ days; Time $4=361-480$ days; Time $5 \geq 481$ ), and six time segments (i.e., Time $1 \leq 90$ days; Time $2=91-180$ days; Time $3=181-270$ days; Time 4 = 271360 days; Time $5=361-450$ days; Time $6 \geq 451$ ), respectively. CAPS scores across assessment occasions were sorted into their corresponding time segments and used as the outcome variable in order to evaluate the time structures. To minimize the loss of data that can result from instances in which participants have two data points that corresponded to the same time segment, the data point closest to an adjacent empty time segment was shifted to that time segment. However, if both data points were more than half the number of days of a time segment away 
from their closest adjacent time segment (i.e., more than 60 days away from the next time segment for the 5 time segment structure and more than 45 days away from the next time segment for the 6 time segment structure), or there was not an empty adjacent time segment, the average of the two data points was used in the existing time segment.

After establishing the best fitting time structure, a series of univariate LDS models were constructed to determine the model that best represented change over time for PTSD (i.e., CAPS and PCL in separate analyses) and dimensions of interpersonal relationship quality (i.e., subscales of the QRI). These analyses evaluated the presence of autoregressive/proportional change as well as non-stationarity/constant change for each variable. Additionally, given that the autoregressive/proportional change can be constrained to be equal across time (i.e., the effect of one's level on a variable at a given assessment on the subsequent assessment stays consistent across assessment occasions) or permitted to vary across time (i.e., the effect of one's level on a variable at a given assessment on the subsequent assessment can change across assessment occasions), these models allowed for the evaluation of the most accurate representation of the autoregressive/proportional change (King, King, McArdle, Saxe et al., 2006).

Six univariate LDS models were constructed for each variable (i.e., CAPS, PCL, QRISupport, -Conflict, -Depth). Figure 2 depicts the path diagrams for the six univariate LDS models that were examined based on a 5 segment time structure. The no change model suggests that there is no change in the variable over time (see Figure 2, panel a). The constant change model, accounts for the non-stationarity/constant change in a variable over time (see Figure 2, panel b). The fixed proportional change model accounts for the autoregressive/proportional change in the variable, but constrains this change to be equal over time (see Figure 2, panel c). The variable proportional change model is similar to the fixed proportional change model, but 
allows the change to vary across time (see Figure 2, panel d). The dual fixed proportional change model accounts for both the non-stationarity/constant change and autoregressive/proportional change in the variable, but constrains the proportional change to be equal over time (see Figure 2, panel e). Finally, the dual variable proportional change model is similar to the dual fixed proportional change model, but allows the proportional change component to vary across time (see Figure 2, panel f). 


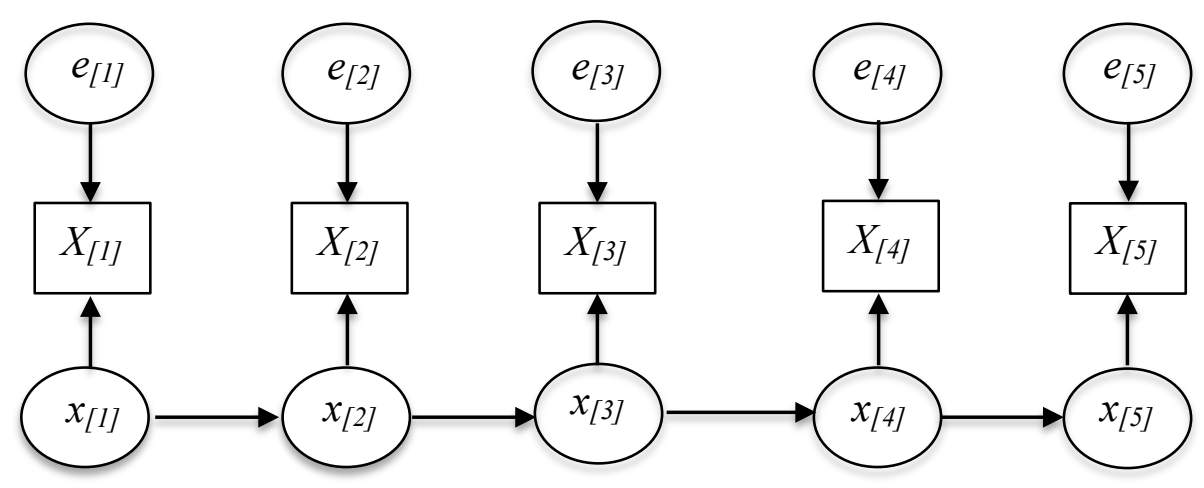

a) No Change Model Path Diagram

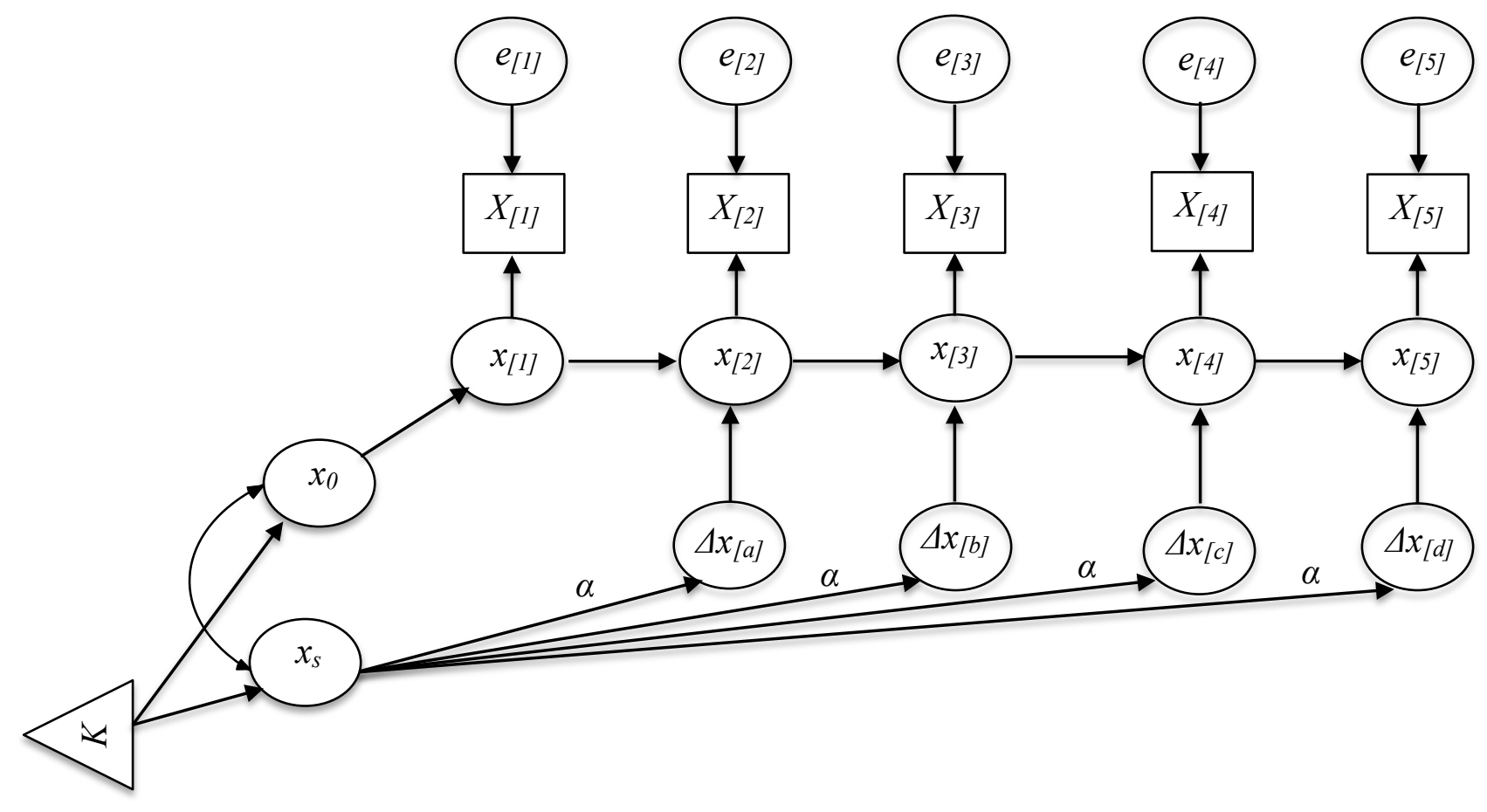

b) Constant Change Model Path Diagram 


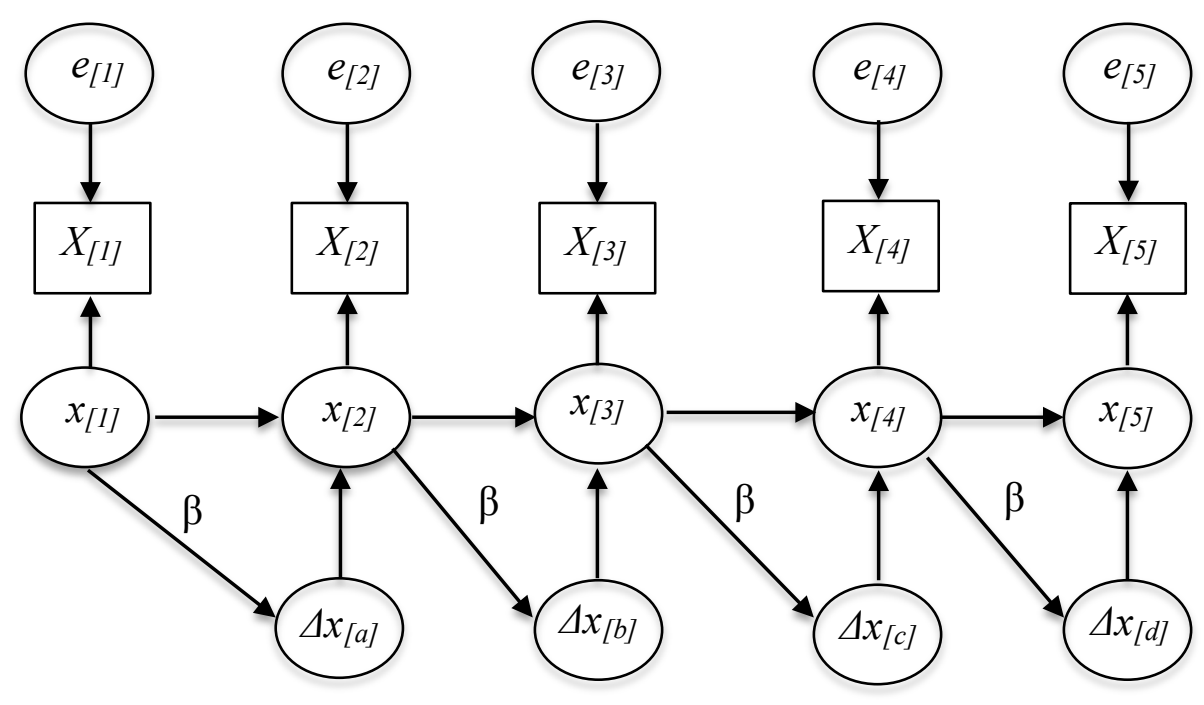

c) Fixed Proportional Change Model Path Diagram

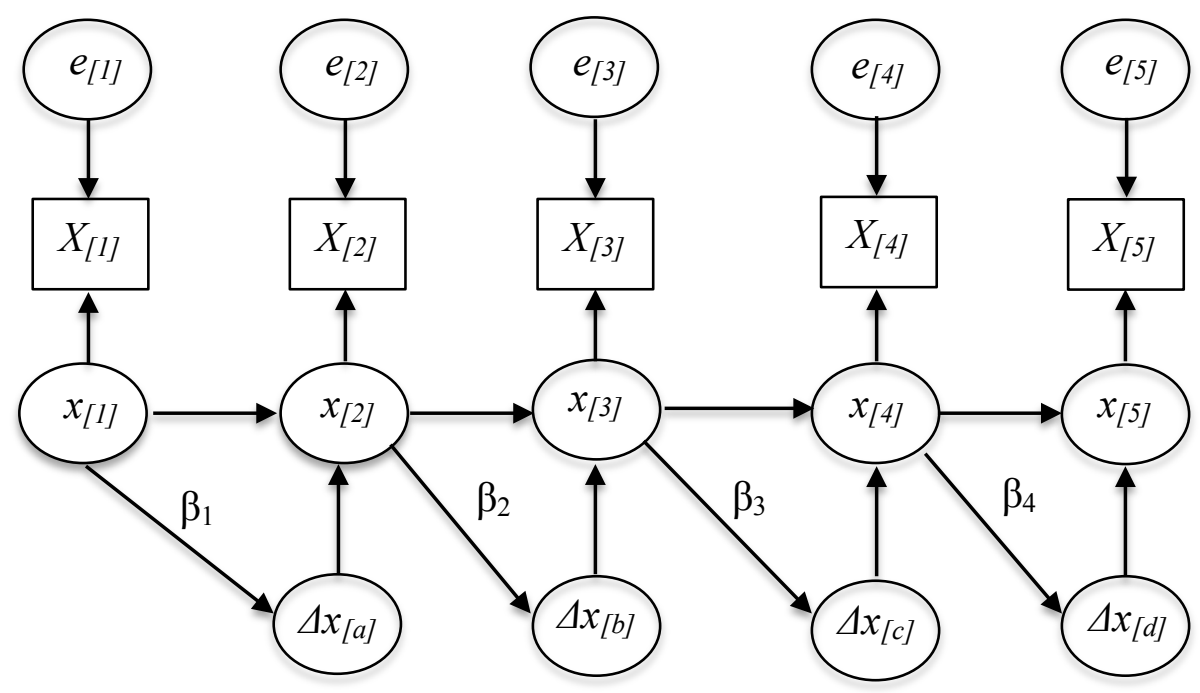

d) Variable Proportional Change Model Path Diagram 


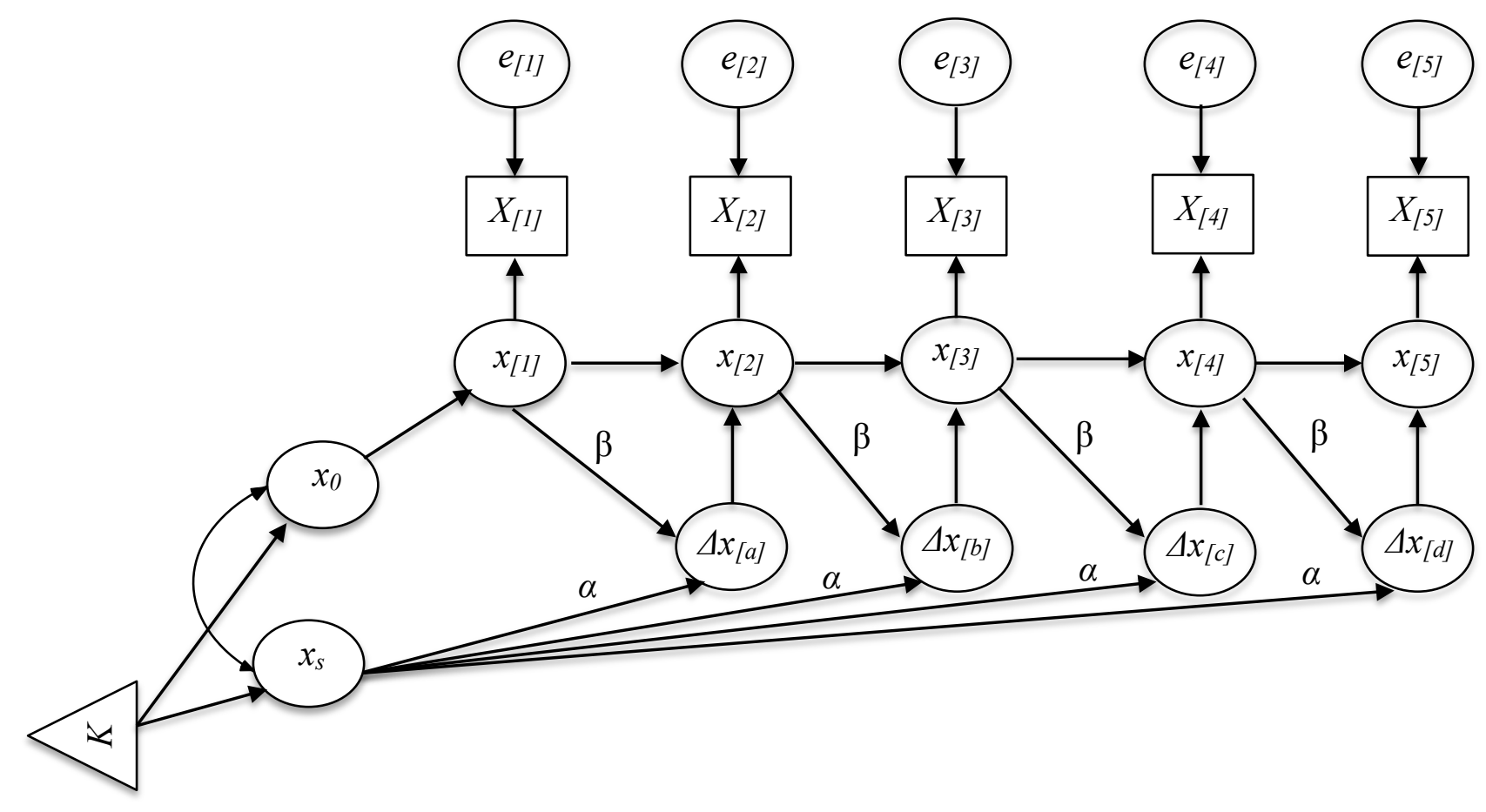

e) Dual Fixed Proportional Change Model Path Diagram

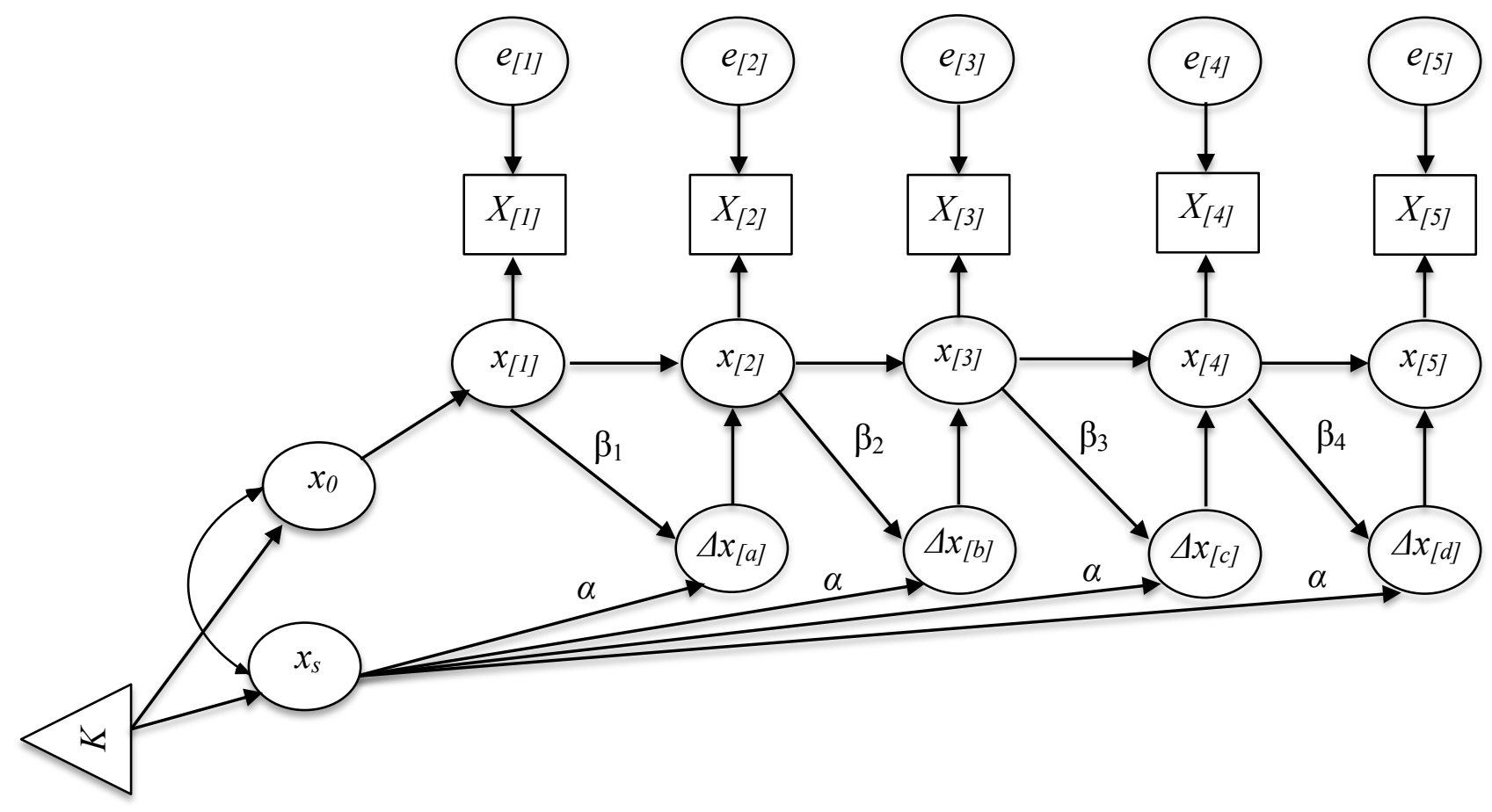

f) Dual Variable Proportional Change Model Path Diagram 
Figure 2. Path diagrams for the six univariate latent difference score (LDS) models based on a 5 segment time structure. Squares represent observed variables, circles represent latent (or unobserved) variables, and the triangle $/ K$ represents a constant used to estimate means and intercepts. Single-headed arrows indicate a regression coefficient, intercept, or mean. Doubleheaded arrows indicate a covariance. $X_{[t]}$ represents the observed variable score at time $t, x_{[t]}$ represents the latent variable score at time $t$, and $\Delta x_{[t]}$ represents the LDS between corresponding time points. $x_{0}$ represents the estimated mean for the initial status of variable $\mathrm{X}, \alpha \times x_{\mathrm{s}}$ represents the non-stationarity/constant change, $\beta_{\mathrm{t}}$ represents the autoregressive/proportional change as a functioning of latent variable $x$ at time $t$ on the subsequent LDS, and $e_{[t]}$ represents the error term at time $t$. Error terms were constrained to be equal. 
Analyses began with the most saturated model for each variable (i.e., the dual variable proportional change model; see Figure 2, panel f), which served as a basis for comparison for each subsequent model. As each subsequent model was constructed, the chi-square difference test $\left(\chi_{\text {diff }}^{2}\right)$ was used to examine if the more parsimonious model resulted in a poorer fit to the data compared to the dual variable proportional change model. The chi-square difference test compares nested models, testing the hypothesis that the reduced model is a poorer fit to the data. A null finding on the chi-square difference test $(p>.05)$ suggests that the more parsimonious model does not result in a poorer fit to the data (Blunch, 2008) and should be accepted over alternative models that are more complex but do not fit the data better. Additionally, the fit of models were evaluated and compared using a number of fit indices, including the chi-square goodness of fit test $\left(\chi^{2}\right)$, the Comparative Fit Index (CFI; Bentler, 1990), the Root Mean Square Error of Approximation (RMSEA; Steiger \& Lind, 1980) and associated significance test, and the Akaike Information Criterion (AIC; Akaike, 1973).

The chi-square goodness of fit test assesses the absolute fit of a model. It evaluates how well the proposed model can recreate the variance-covariance matrix of the data compared to the one created by no model. The null hypothesis associated with chi-square goodness of fit test is that the proposed model fits the data. Accordingly, a null finding $(p>.05)$ suggests good model fit. The chi-square goodness of fit test is often influenced by sample size, with a tendency to reject the model (i.e., a significant finding) among large samples (Tabachnick \& Fidell, 2007). In contrast, the CFI is a relative fit index, meaning that is compares the proposed model to a saturated model (i.e., a model with the maximum number of parameters) and an independence model (i.e., a model with no correlations among variables). The saturated and independence models provide boundaries confining the model being examined. Given that the saturated model 
provides the best possible fit to the data (i.e., $\mathrm{CFI}=1.00$ ), and the independence model provides the worst possible fit to the data (i.e., $\mathrm{CFI}=.00$ ), relative fit indices indicate the distance that the proposed model has travelled between these boundaries. The CFI takes into account the degrees of freedom associated with each model, making it appropriate for smaller samples and for comparing models to assess model fit (Bentler, 1990; Blunch, 2008). Values greater than .95 are indicative of good model fit (Blunch, 2008). The RMSEA is an additional fit index, for which $.01, .05$, and .08 represent an excellent, good, and moderate fit, respectively (MacCallum, Browne, \& Sugawara, 1996; Steiger \& Lind, 1980). The RMSEA is used with its associated significance test, which tests the null hypothesis that the population RMSEA is less than .05 . Accordingly, a null finding $(p>.05)$ is indicative of good model fit (Blunch, 2008). Finally, the AIC, is a comparative index that does not require models to be nested, which rewards parsimonious models by taking into account the associated degrees of freedom. Smaller values indicate a better fitting model when comparing competing models (Akaike, 1973; Blunch, 2008).

After evaluating the six univariate models for each variable, the best fitting models were selected for each variable in order to construct bivariate LDS models. Bivariate LDS modeling is a framework used to examine two univariate LDS models simultaneously. It is used to assess the dynamic interplay between two variables over time. Specifically, it allows for the examination of cross-lagged effects from one variable to the other (e.g., the effect of PTSD at one time point on change in interpersonal relationship functioning from that time point to the next, and vice versa) while controlling for the two internal sources of change within each variable (i.e., autoregressive/proportional change and non-stationarity/constant change).

Six bivariate LDS models were constructed using the best fitting univariate models in combinations: 1) CAPS model with QRI-Support model, 2) CAPS model with QRI-Conflict 
model, 3) CAPS model with QRI-Depth model, 4) PCL model with QRI-Support model, 5) PCL model with QRI-Conflict model, and 6) PCL model with QRI-Depth model. As mentioned above, the bivariate LDS approach to modeling longitudinal data has the added benefit of accounting for autoregressive/proportional change and non-stationarity/constant change, which are not fully accounted for in cross-lagged panel designs (King, King, McArdle, Saxe et al., 2006). The chi-square goodness of fit test, RMSEA and associated significance test, and CFI were used to evaluate the fit of each bivariate LDS model. Additionally, parameter estimates for each bivariate LDS model were examined to evaluate the significance of the autoregressive/proportional change, non-stationarity/constant change, and cross-lagged effects within the bivariate context.

\section{Results}

Means and standard deviations for the CAPS, PCL, and QRI subscales for the total sample at each assessment point can be found in Table 2. Correlations between the CAPS, PCL, and QRI subscales at each assessment point, as well as the means and standard deviations for the CAPS, PCL, and QRI subscales separated by type of trauma (i.e., interpersonal vs. noninterpersonal) and type of close other (intimate vs. non-intimate) can be found in Table 3. Interestingly, with the exception of the correlation between PCL and QRI-Conflict scores at the initial assessment $(r=.31, p<.01)$, all correlations between CAPS and QRI scores as well as PCL and QRI scores at the initial assessment were small and not statistically significant $(r \mathrm{~s}$ ranged from -.16 to $.17, p \mathrm{~s}>.05)$. 
Table 2

Means and Standard Deviations for Outcome Variables across Assessment Points.

\begin{tabular}{|c|c|c|c|c|c|c|c|c|c|c|c|c|}
\hline \multirow[b]{2}{*}{ Variable } & \multicolumn{3}{|c|}{ First Assessment } & \multicolumn{3}{|c|}{ Second Assessment } & \multicolumn{3}{|c|}{ Third Assessment } & \multicolumn{3}{|c|}{ Fourth Assessment } \\
\hline & $n$ & $M$ & $S D$ & $n$ & $M$ & $S D$ & $n$ & $M$ & $S D$ & $n$ & $M$ & $S D$ \\
\hline CAPS & 107 & 43.50 & 26.36 & 92 & 31.23 & 26.94 & 91 & 22.55 & 24.00 & 85 & 17.39 & 24.93 \\
\hline PCL & 106 & 42.10 & 14.83 & 91 & 36.10 & 14.74 & 92 & 31.77 & 14.36 & 82 & 29.87 & 14.37 \\
\hline QRI-Support & 101 & 3.12 & .62 & 91 & 2.97 & .80 & 90 & 3.03 & .79 & 80 & 2.94 & .83 \\
\hline QRI-Conflict & 100 & 1.89 & .62 & 92 & 1.84 & .65 & 90 & 1.80 & .60 & 82 & 1.88 & .60 \\
\hline QRI-Depth & 101 & 3.08 & .71 & 92 & 2.96 & .81 & 90 & 2.97 & .77 & 82 & 2.89 & .86 \\
\hline
\end{tabular}

Note. CAPS = Clinician-Administered PTSD Scale; PCL = PTSD Checklist; QRI = Quality of Relationships Inventory. 
Table 3

Correlations between Outcomes Variables, and Means and Standard Deviations for Outcome Variables Separated by Trauma Type and Close Other Type across Assessment Points.

\begin{tabular}{|c|c|c|c|c|c|c|c|c|c|c|c|c|c|c|c|c|c|c|c|c|c|}
\hline & & 1. & 2. & 3. & 4. & 5. & 6. & 7. & 8. & 9. & 10. & 11. & 12. & 13. & 14. & 15. & 16. & 17. & 18. & 19. & 20. \\
\hline 1. & T1 CAPS & - & & & & & & & & & & & & & & & & & & & \\
\hline 2. & T2 CAPS & $.75^{* * *}$ & - & & & & & & & & & & & & & & & & & & \\
\hline 3. & T3 CAPS & $.65^{* * *}$ & $.80^{* * *}$ & - & & & & & & & & & & & & & & & & & \\
\hline 4. & T4 CAPS & $.62^{2 * *}$ & $.81^{3 * *}$ & $.83^{* * * *}$ & - & & & & & & & & & & & & & & & & \\
\hline 5. & T1 PCL & $.80^{* * *}$ & $.68^{* * *}$ & $.61^{* * * *}$ & $.47^{* * *}$ & - & & & & & & & & & & & & & & & \\
\hline 6. & T2 PCL & $.67^{* * *}$ & $.83^{* * *}$ & $.69^{* * * *}$ & $.63^{* * *}$ & $.76^{* * *}$ & - & & & & & & & & & & & & & & \\
\hline 7. & T3 PCL & $.52^{2 * *}$ & $.65^{5 * *}$ & $.78^{* * *}$ & $.60^{* * *}$ & $.57^{* * *}$ & $.68^{* * *}$ & - & & & & & & & & & & & & & \\
\hline 8. & T4 PCL & $.67^{* * *}$ & $.79^{4 * s}$ & $.79^{* * * *}$ & $.84^{* * *}$ & $.59^{* * *}$ & $.70^{* * * *}$ & $.71^{* * *}$ & - & & & & & & & & & & & & \\
\hline 9. & T1 QRI-S & -.16 & -.08 & -.09 & -.21 & -.06 & -.14 & -.08 & $-.29^{*}$ & - & & & & & & & & & & & \\
\hline 10. & T2 QRI-S & -.19 & -.09 & -.13 & -.17 & -.09 & -.08 & -.05 & $-.23^{*}$ & $.62^{* * *}$ & - & & & & & & & & & & \\
\hline 11. & T3 QRI-S & $-.22^{*}$ & -.10 & -.03 & -.14 & -.11 & -.07 & -.12 & -.18 & $.56^{* * *}$ & $.66^{* * *}$ & - & & & & & & & & & \\
\hline 12. & T4 QRI-S & -.17 & -.03 & -.03 & -.03 & -.13 & .03 & -.03 & -.10 & $.49^{* * *}$ & $.57^{* * *}$ & $.73^{* * *}$ & - & & & & & & & & \\
\hline 13. & T1 QRI-C & .17 & $.31^{* *}$ & $.26^{*}$ & -.18 & $.31^{* *}$ & $.38^{* * *}$ & .19 & .20 & $-.27^{* *}$ & -.21 & -.14 & -.09 & - & & & & & & & \\
\hline & T2 QRI-C & .17 & $.27^{*}$ & .12 & .21 & $.23^{*}$ & $.34^{* *}$ & .08 & .16 & $-.26^{*}$ & -.15 & $-.26^{*}$ & -.13 & $.53^{* * *}$ & - & & & & & & \\
\hline & T3 QRI-C & .14 & .19 & $.24^{*}$ & .14 & $.22^{*}$ & $.30^{* *}$ & .18 & .12 & $-.22^{*}$ & $-.32^{* *}$ & $-.31^{* *}$ & -.17 & $.59^{* * *}$ & $.69^{* * *}$ & - & & & & & \\
\hline & T4 QRI-C & .19 & $.27^{*}$ & $.24^{*}$ & .21 & .12 & $.27^{*}$ & .20 & $.23^{*}$ & -.21 & $-.24^{*}$ & $-.31^{* *}$ & -.07 & $.58^{* * *}$ & $.64^{* * *}$ & $.64^{* *}$ & - & & & & \\
\hline & T1 QRI-D & -.00 & .06 & .06 & -.05 & .10 & -.02 & .03 & -.09 & $.75^{* * * *}$ & $.56^{* * *}$ & $.53^{* * *}$ & $.53^{* * *}$ & .02 & -.13 & -.15 & -.06 & - & & & \\
\hline 18. & T2 QRI-D & -.04 & .10 & .06 & .04 & .07 & .09 & .03 & -.07 & $.51^{* * *}$ & $.86^{* * *}$ & $.66^{* * *}$ & $.60^{* * *}$ & .03 & .07 & -.15 & -.11 & $.68^{* * *}$ & - & & \\
\hline 19. & T3 QRI-D & -.12 & -.01 & .10 & .04 & .02 & .06 & .01 & -.06 & $.53^{* * *}$ & $.54^{* * *}$ & $.83^{* * *}$ & $.64^{* * *}$ & .01 & -.11 & -.06 & -.14 & $.63^{* * *}$ & $.70^{* * * *}$ & - & \\
\hline \multirow{3}{*}{\multicolumn{2}{|c|}{ 20. T4 QRI-D }} & -.03 & .08 & .09 & .12 & .02 & .11 & .10 & .03 & $.44^{* * * *}$ & $.49^{* * *}$ & $.62^{* * *}$ & $.89^{* * *}$ & .10 & -.04 & -.08 & .02 & $.60^{* * *}$ & $.62^{* * *}$ & $.69^{* * * *}$ & - \\
\hline & & $M$ & $M$ & $M$ & $M$ & $M$ & $M$ & $M$ & $M$ & $M$ & $M$ & $M$ & $M$ & $M$ & $M$ & $M$ & $M$ & $M$ & $M$ & $M$ & $M$ \\
\hline & & (SD) & (SD) & $(S D)$ & (SD) & (SD) & (SD) & (SD) & (SD) & $(S D)$ & $(S D)$ & $(S D)$ & $(S D)$ & $(S D)$ & (SD) & (SD) & (SD) & (SD) & (SD) & (SD) & (SD) \\
\hline \multicolumn{22}{|c|}{ Trauma Type } \\
\hline \multirow{2}{*}{\multicolumn{2}{|c|}{ Interpersonal }} & 51.71 & 39.85 & 28.50 & 23.90 & 44.89 & 38.33 & 34.85 & 33.37 & 3.12 & 2.79 & 2.93 & 2.77 & 1.88 & 1.76 & 1.77 & 1.92 & 3.05 & 2.79 & 2.85 & 2.74 \\
\hline & & $(24.75)$ & (26.75) & (24.30) & (24.73) & (14.74) & (14.84) & (14.64) & $(14.69)$ & (.64) & $(.85)$ & $(.80)$ & (.79) & $(.62)$ & $(.61)$ & $(.56)$ & $(.57)$ & $(.67)$ & $(.87)$ & $(.73)$ & $(.80)$ \\
\hline \multirow{2}{*}{\multicolumn{2}{|c|}{ Non-Interpersonal }} & 34.81 & 22.22 & 16.98 & 11.60 & 39.19 & 33.60 & 28.82 & 26.86 & 3.12 & 3.12 & 3.13 & 3.10 & 1.90 & 1.93 & 1.83 & 1.84 & 3.11 & 3.14 & 3.08 & 3.02 \\
\hline & & $(25.40)$ & $(24.30)$ & $(22.57)$ & $(23.91)$ & $(14.49)$ & $(14.40)$ & $(13.59)$ & $(13.53)$ & $(.62)$ & $(.69)$ & $(.78)$ & $(.85)$ & $(.62)$ & $(.70)$ & $(.64)$ & $(.63)$ & $(.76)$ & $(.69)$ & $(.80)$ & $(.90)$ \\
\hline \multicolumn{22}{|c|}{ Close Other Type } \\
\hline \multirow{2}{*}{\multicolumn{2}{|c|}{ Intimate }} & 44.38 & 34.91 & 23.00 & 18.95 & 43.33 & 39.05 & 32.40 & 29.74 & 3.40 & 3.45 & 3.65 & 3.45 & 2.07 & 1.84 & 1.78 & 1.83 & 3.41 & 3.44 & 3.54 & 3.48 \\
\hline & & $(26.05)$ & $(27.33)$ & (23.01) & (17.88) & (14.93) & (15.58) & $(15.08)$ & (11.75) & (.64) & $(.61)$ & (.39) & (.59) & (.77) & (.69) & (.53) & $(.61)$ & $(.58)$ & $(.57)$ & (.42) & $(.56)$ \\
\hline
\end{tabular}


$=$ First Assessment; $\mathrm{T} 2=$ Second Assessment; $\mathrm{T} 3=$ Third Assessment; $\mathrm{T} 4=$ Fourth Assessment. " $p<.05 . " p<.01 . " * p<.001$ 
Due to study attrition, only 85 , of the 107 participants included in the study completed the interview portion of their fourth assessment. This significantly reduced the sample size for analyses in which end-state PTSD diagnostic status was required as a predictor variable. In order to prevent the deletion of these participants for the respective analyses, an alternative approach was utilized to estimate end-state PTSD for individuals within the sample. Specifically, growth curve modeling was used to estimate end-state PTSD symptom severity (i.e., projected PTSD symptom severity at the final assessment point) by reverse coding time and centering it at the average number of days posttrauma at which those who completed their fourth assessment were assessed. With time coded such that zero represents the average amount of days posttrauma at the fourth assessment, the Level-1 regression intercept can be interpreted as an estimate of the level of the outcome variable (PTSD severity) at this time for each participant. These Level-1 coefficients can be saved and used in subsequent analyses (see Griffin, 1997; Iverson et al., 2011; Walling, Suvak, Howard, Taft, \& Murphy, 2012). This created a unique estimate of projected end-state PTSD symptom severity for each participant based on the available data from the trajectory of their PTSD symptoms over time based on the CAPS $(M=17.40, S D=21.91)$. This offers two advantages: 1) it provides estimates of end-state PTSD severity for participants who did not complete the fourth assessment, and 2) the estimates of end-state functioning are based on all of the data present, overcoming some of the reliability issues with basing an estimate on only one score. In order to preserve the variance in the projected end-state PTSD symptom severity variable that was created, the variable was left as a continuous variable rather than dichotomizing it at a severity indicative of a probable PTSD diagnosis. This is consistent with taxometric research suggesting that PTSD is better conceptualized as a dimensional construct rather than a discrete diagnostic category (e.g., Broman-Fulks, Ruggiero, Green, \& 
Kilpatrick, 2006). Projected end-state PTSD symptom severity was mean centered to make the intercept terms more interpretable (i.e., estimate when end-state PTSD was held constant at its mean). Accordingly, for all analyses for which the use end-state PTSD diagnostic status was originally proposed, projected end-state PTSD symptom severity was used in its place.

\section{$\operatorname{Aim} 1$}

The intercept term for the model examining the trajectory of CAPS scores over time indicated that, on average, participants' score on the CAPS was initially approximately $50(b=$ $50.19, S E=2.91, t(106)=17.23, p<.001, d=3.35)$ and that CAPS scores significantly decreased over time $(b=-.06, S E=.01, t(106)=-11.01, p<.001, d=-2.14)$. Similarly, the model examining the trajectory of PCL scores over time indicated that participants score on the PCL was initially approximately 45 on average $(b=45.27, S E=1.64, t(105)=27.60, p<.001, d$ $=5.39)$ and that PCL scores significantly decreased over time $(b=-.03, S E=.00, t(105)=-9.06$, $p<.001, d=-1.77)$.

Results of the models using projected end-state PTSD symptom severity as a Level-2 predictor revealed a significant positive effect of projected end-state PTSD symptom severity on initial PTSD symptom severity (i.e., intercept) for both the CAPS and PCL. Additionally, there was a significant effect of projected end-state PTSD symptom severity on symptom trajectory over time (i.e., slope) for the PCL but not the CAPS, such that end-state PTSD symptom severity decreased, as the slope of self-reported PTSD symptoms over time became steeper (see Table 4 and Figure 3). 
Table 4

Growth Curve Models Predicting Initial PTSD Symptom Severity and Symptom Trajectory from Projected End-State PTSD Severity.

\begin{tabular}{|c|c|c|c|c|c|c|}
\hline \multicolumn{7}{|c|}{ CAPS } \\
\hline Effect & $b$ & $S E$ & $t$ & $d f$ & $p$ & $d$ \\
\hline Initial CAPS Severity (intercept) & 50.24 & 2.19 & 22.90 & 105 & $<.001$ & 4.47 \\
\hline End-State PTSD & .89 & .13 & 7.10 & 105 & $<.001$ & 1.39 \\
\hline CAPS Trajectory (slope) & -.06 & .01 & -12.38 & 105 & $<.001$ & -2.42 \\
\hline End-State PTSD & .00 & .00 & 1.58 & 105 & .117 & .31 \\
\hline \multicolumn{7}{|c|}{ PCL } \\
\hline Effect & $b$ & $S E$ & $t$ & $d f$ & $p$ & $d$ \\
\hline Initial PCL Severity (intercept) & 45.11 & 1.41 & 31.95 & 104 & $<.001$ & 6.27 \\
\hline End-State PTSD & .38 & .07 & 5.26 & 104 & $<.001$ & 1.03 \\
\hline PCL Trajectory (slope) & -.03 & .00 & -9.29 & 104 & $<.001$ & -1.82 \\
\hline End-State PTSD & .00 & .00 & 2.51 & 104 & .014 & .49 \\
\hline \multicolumn{7}{|c|}{ Note. End-state PTSD symptom severity was mean centered. CAPS = Clinician-Administered } \\
\hline PTSD Scale; End-State PTSD = 1 & end-stat & TSD s & imptom & verit & based $\mathrm{c}$ & the \\
\hline
\end{tabular}




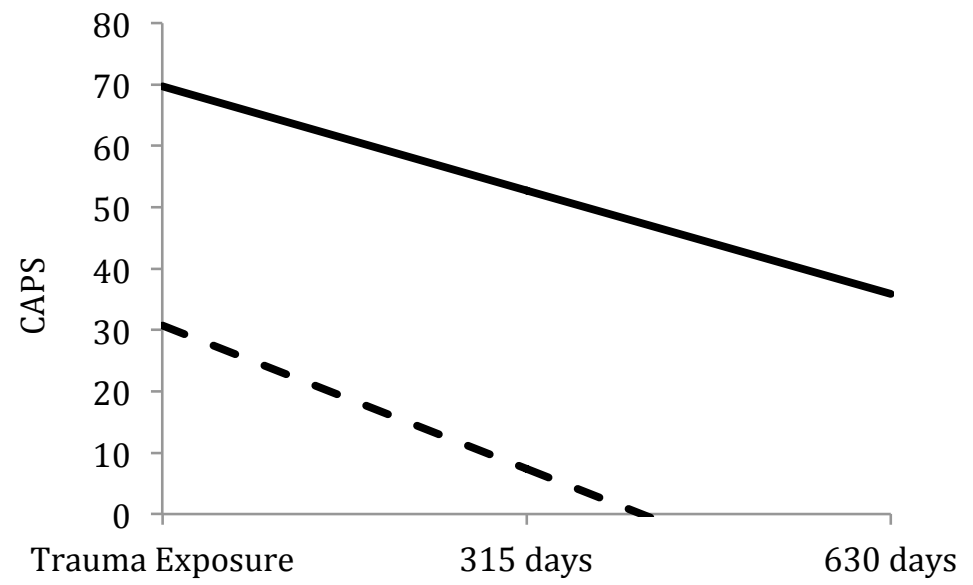

End-State PTSD +1SD — - End-State PTSD -1SD

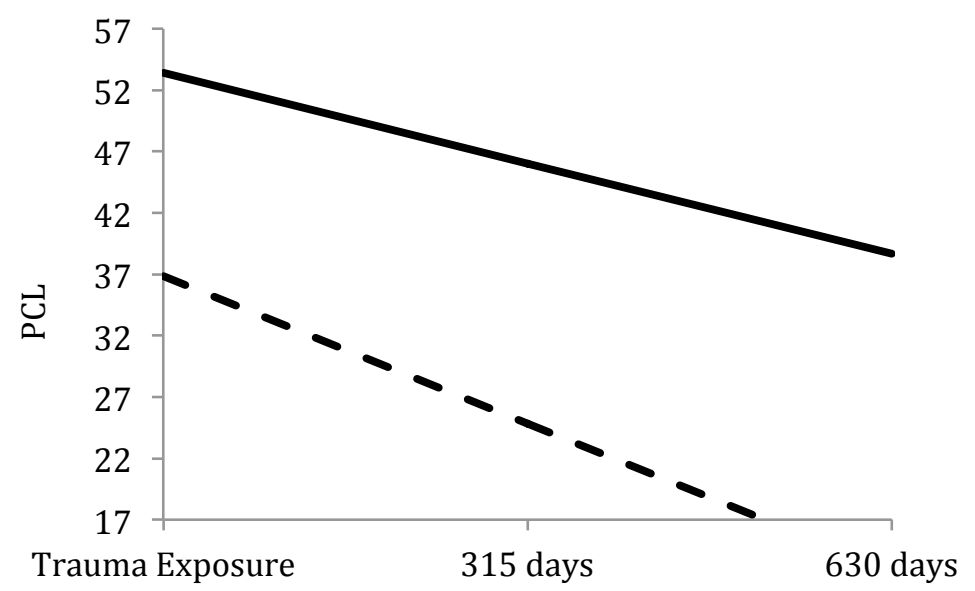

—End-State PTSD +1SD - - End-State PTSD -1SD

Figure 3. Trajectories of PTSD symptoms over time according to the CAPS and PCL across high and low levels of projected end-state PTSD. Trajectories are depicted at 1 standard deviation above and below the mean of the projected end-state PTSD to facilitate interpretation. Note. CAPS = Clinician-Administered PTSD Scale; End-State PTSD = projected end-state PTSD symptom severity based on the CAPS; PCL = PTSD Checklist. 


\section{$\operatorname{Aim} 2$}

The model examining the trajectory of QRI-Support indicated that participants had an average initial QRI-Support score of $3.13(b=3.13, S E=.08, t(105)=41.34, p<.001, d=8.07)$, and that, although it appears to be decreasing over time, the slope of QRI-Support just failed to meet conventional levels of significance $(b=-.00, S E=.00, t(105)=-1.97, p=.050, d=-.39)$. The model examining QRI-Conflict over time indicated that participants had an average initial QRI-Conflict score of $1.90(b=1.90, S E=.07, t(105)=27.40, p<.001, d=5.35)$, but that QRIConflict scores did not significantly change over time $(b=-.00, S E=.00, t(105)=-.84, p=.405$, $d=$-.16). Finally, the model examining QRI-Depth over time indicated that participants had an average initial QRI-Depth score of $3.10(b=3.10, S E=.08, t(105)=38.88, p<.001, d=7.59)$, and that QRI-Depth significantly decreased over time $(b=-.00, S E=.00, t(105)=-2.26, p=$ $.026, d=-.44)$.

Results of the above models using projected end-state PTSD symptom severity as a Level2 predictor indicated a significant effect of projected end-state PTSD symptom severity on initial status (i.e., intercept) for QRI-Conflict and a marginally significant effect on QRI-Support; the effect on QRI-Depth was not significant. Specifically, as projected end-state PTSD symptom severity increased, initial relationship conflict increased and initial relationship support decreased. Notably, there was not a significant effect of projected end-state PTSD symptom severity on the trajectory (i.e., slope) of any of the QRI subscales, (see Table 5 and Figure 4). 
Table 5

Growth Curve Models Predicting Interpersonal Relationship Functioning Initial Status and Trajectory from Projected End-State PTSD Severity.

\begin{tabular}{|c|c|c|c|c|c|c|}
\hline \multicolumn{7}{|c|}{ QRI-Support } \\
\hline Effect & $b$ & $S E$ & $t$ & $d f$ & $p$ & $d$ \\
\hline Initial Status QRI-Support (intercept) & 3.13 & .07 & 41.96 & 104 & $<.001$ & 8.23 \\
\hline End-State PTSD & -.01 & .00 & -1.86 & 104 & .066 & -.36 \\
\hline QRI-Support Trajectory (slope) & -.00 & .00 & -1.97 & 104 & .052 & -.39 \\
\hline End-State PTSD & .00 & .00 & 1.45 & 104 & .151 & .28 \\
\hline \multicolumn{7}{|c|}{ QRI-Conflict } \\
\hline Effect & $b$ & $S E$ & $t$ & $d f$ & $p$ & $d$ \\
\hline Initial Status QRI-Conflict (intercept) & 1.90 & .07 & 28.16 & 104 & $<.001$ & 5.52 \\
\hline End-State PTSD & .01 & .00 & 2.62 & 104 & .010 & .51 \\
\hline QRI-Conflict Trajectory (slope) & -.00 & .00 & -.84 & 104 & .403 & -.16 \\
\hline End-State PTSD & -.00 & .00 & -.47 & 104 & .642 & -.09 \\
\hline \multicolumn{7}{|c|}{ QRI-Depth } \\
\hline Effect & $b$ & $S E$ & $t$ & $d f$ & $p$ & $d$ \\
\hline Initial Status QRI-Depth (intercept) & 3.10 & .08 & 38.86 & 104 & $<.001$ & 7.62 \\
\hline End-State PTSD & .00 & .00 & .22 & 104 & .829 & .04 \\
\hline QRI-Depth Trajectory (slope) & -.00 & .00 & -2.23 & 104 & .028 & -.44 \\
\hline End-State PTSD & .00 & .00 & 1.25 & 104 & .214 & .25 \\
\hline
\end{tabular}

Note. End-state PTSD symptom severity was mean centered. End-State PTSD = projected endstate PTSD symptom severity based on the Clinician-Administered PTSD Scale, QRI = Quality 
of Relationships Inventory. 


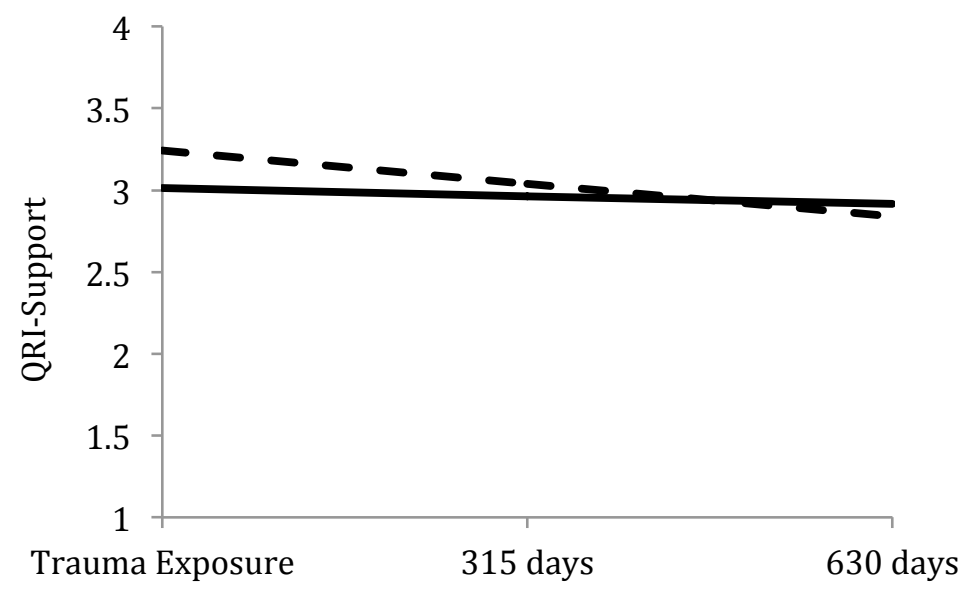

- End-State PTSD +1SD $\quad$ - End-State PTSD -1SD

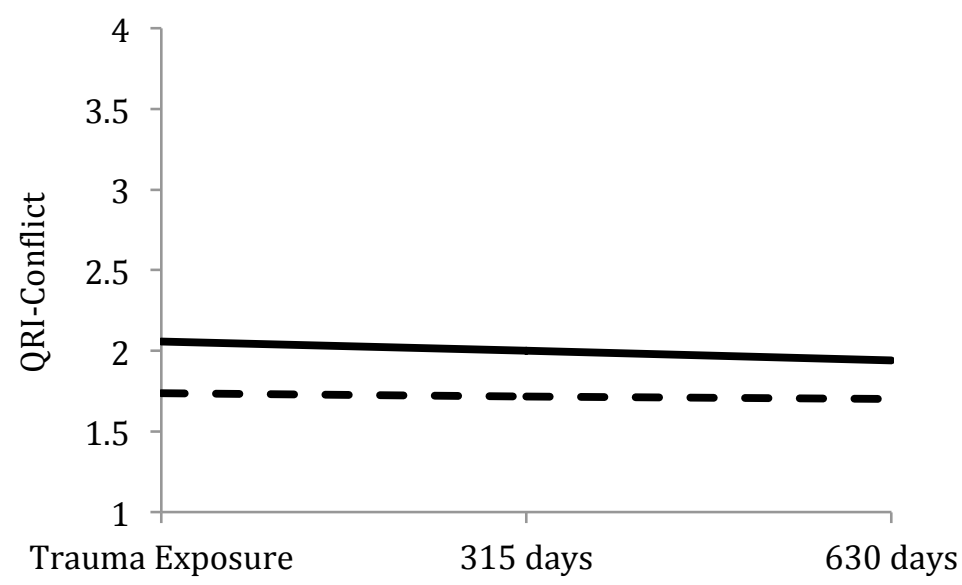

—End-State PTSD +1SD - - End-State PTSD -1SD

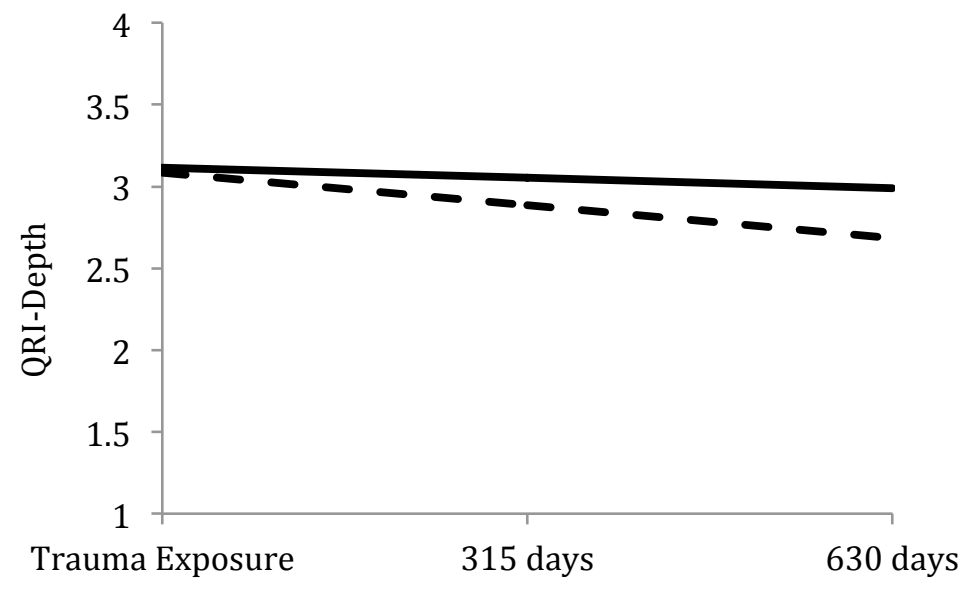

— End-State PTSD +1SD — - End-State PTSD -1SD 
Figure 4. Trajectories of the QRI subscales across high and low levels of projected end-state PTSD. Trajectories are depicted at 1 standard deviation above and below the mean of the projected end-state PTSD for interpretation.

Note. End-State PTSD = projected end-state PTSD symptom severity based on the ClinicianAdministered PTSD Scale; QRI = Quality of Relationships Inventory. 


\section{$\operatorname{Aim} 3$}

Results of the models predicting interpersonal relationship functioning using time, projected end-state PTSD symptom severity, trauma type (interpersonal vs. non-interpersonal), and the interaction of projected end-state PTSD symptom severity by trauma type are depicted in Table 6. When entered without the interaction (Step 1), consistent with the findings in Aim 2, there was a marginally significant effect of projected end-state PTSD symptom severity on the initial status (i.e., intercept) for QRI-Support and significant effect for QRI-Conflict; the effect on QRI-Depth was not significant. Notably, initial status on all of the QRI subscales did not significantly differ across trauma types. When considering the trajectory (i.e., slope) of relationship functioning over time, unlike the results in Aim 2, there was a significant effect of projected end-state PTSD symptom severity on both the trajectory of QRI-Support and -Depth, but not -Conflict. More specifically, increases in end-state PTSD symptom severity were associated with improvements in QRI-Support and -Depth over time. Additionally, there was a marginally significant effect of trauma type on the trajectory of QRI-Support and -Depth, but not -Conflict, such that the course of QRI-Support and -Depth was poorer for individuals exposed to interpersonal traumas compared to those exposed to non-interpersonal traumas.

After the interaction was added to the model (Step 2), results indicated that there was a significant end-state PTSD symptom severity by trauma type interaction effect on the initial status of QRI-Support and -Depth, but not Conflict. These findings suggest that the effect of endstate PTSD symptom severity on initial QRI-Support and -Depth, differed between those exposed to interpersonal compared with those exposed to non-interpersonal traumas. Post-hoc analyses, conducted to probe and further interpret these interactions, suggested that there was a significant negative association between projected end-state PTSD symptom severity and initial 
QRI-Support for individuals who experienced non-interpersonal traumas $b=-.01, S E=.00$, $t(102)=-3.79, p<.001, d=-.75$, but not those exposed to interpersonal traumas, $b=-.00, S E=$ $.00, t(102)=-.07, p=.948, d=-.01$. Additionally, there was a marginally significant positive association between projected end-state PTSD symptom severity and initial QRI-Depth for those who experienced interpersonal traumas, $b=.01, S E=.00, t(102)=1.75, p=.082, d=.35$, but a null association for those who experienced non-interpersonal traumas, $b=-.01, S E=.00, t(102)$ $=-1.24, p=.217, d=-.25$. In contrast, the effect of the end-state PTSD symptom severity by trauma type interaction was not significant for the trajectory of any of the QRI subscales, suggesting that the effect of end-state PTSD symptom severity on the trajectory of interpersonal relationship functioning did not differ across types of trauma exposure (see Figure 5). 
Table 6

Growth Curve Models Predicting Interpersonal Relationship Functioning Initial Status and

Trajectory by Projected End-State PTSD Severity, Trauma Type, and their Interaction.

\begin{tabular}{|c|c|c|c|c|c|c|}
\hline \multicolumn{7}{|c|}{ QRI-Support } \\
\hline \multicolumn{7}{|c|}{ Step 1} \\
\hline Effect & $B$ & $S E$ & $t$ & $d f$ & $p$ & $d$ \\
\hline Initial Status QRI-Support (intercept) & 3.11 & .10 & 29.71 & 103 & $<.001$ & 5.85 \\
\hline End-State PTSD & -.01 & .00 & -1.83 & 103 & .070 & -.36 \\
\hline Trauma Type & .03 & .16 & .17 & 103 & .862 & .03 \\
\hline QRI-Support Trajectory (slope) & .00 & .00 & .01 & 103 & .996 & .00 \\
\hline End-State PTSD & .00 & .00 & 2.26 & 103 & .026 & .44 \\
\hline Trauma Type & -.00 & .00 & -1.95 & 103 & .054 & -.38 \\
\hline \multicolumn{7}{|c|}{ Step 2} \\
\hline Effect & $B$ & $S E$ & $t$ & $d f$ & $p$ & $d$ \\
\hline Initial Status QRI-Support (intercept) & 3.07 & .10 & 30.13 & 102 & $<.001$ & 5.97 \\
\hline End-State PTSD & -.01 & .00 & -3.79 & 102 & $<.001$ & -.75 \\
\hline Trauma Type & .04 & .15 & .27 & 102 & .791 & .05 \\
\hline Interaction & .01 & .01 & 2.28 & 102 & .024 & .45 \\
\hline QRI-Support Trajectory (slope) & -.00 & .00 & -.02 & 102 & .987 & -.00 \\
\hline End-State PTSD & .00 & .00 & 1.71 & 102 & .090 & .34 \\
\hline Trauma Type & -.00 & .00 & -1.97 & 102 & .051 & -.39 \\
\hline Interaction & .00 & .00 & .07 & 102 & .944 & .01 \\
\hline
\end{tabular}




\begin{tabular}{|c|c|c|c|c|c|c|}
\hline \multicolumn{7}{|c|}{ QRI-Conflict } \\
\hline \multicolumn{7}{|c|}{ Step 1} \\
\hline Effect & $B$ & $S E$ & $t$ & $d f$ & $p$ & $d$ \\
\hline Initial Status QRI-Conflict (intercept) & 1.99 & .10 & 19.42 & 103 & $<.001$ & 3.83 \\
\hline End-State PTSD & .01 & .00 & 3.09 & 103 & .003 & .61 \\
\hline Trauma Type & -.19 & .13 & -1.40 & 103 & .163 & -.28 \\
\hline QRI-Conflict Trajectory (slope) & -.00 & .00 & -.82 & 103 & .414 & -.16 \\
\hline End-State PTSD & -.00 & .00 & -.51 & 103 & .612 & -.10 \\
\hline Trauma Type & .00 & .00 & .35 & 103 & .726 & .07 \\
\hline \multicolumn{7}{|c|}{ Step 2} \\
\hline Effect & $B$ & $S E$ & $t$ & $d f$ & $p$ & $d$ \\
\hline Initial Status QRI-Conflict (intercept) & 2.00 & .10 & 19.94 & 102 & $<.001$ & 3.95 \\
\hline End-State PTSD & .01 & .00 & 2.98 & 102 & .004 & .59 \\
\hline Trauma Type & -.19 & .13 & -1.43 & 102 & .157 & -.28 \\
\hline Interaction & -.00 & .01 & -.58 & 102 & .563 & -.11 \\
\hline QRI-Conflict Trajectory (slope) & -.00 & .00 & -.56 & 102 & .576 & -.11 \\
\hline End-State PTSD & .00 & .00 & .32 & 102 & .749 & .06 \\
\hline Trauma Type & .00 & .00 & .32 & 102 & .753 & .06 \\
\hline Interaction & -.00 & .00 & -.90 & 102 & .372 & -.18 \\
\hline
\end{tabular}




\begin{tabular}{|c|c|c|c|c|c|c|}
\hline \multicolumn{7}{|c|}{ QRI-Depth } \\
\hline \multicolumn{7}{|c|}{ Step 1} \\
\hline Effect & $B$ & $S E$ & $t$ & $d f$ & $p$ & $d$ \\
\hline Initial Status QRI-Depth (intercept) & 3.15 & .12 & 25.84 & 103 & $<.001$ & 5.09 \\
\hline End-State PTSD & .00 & .00 & .37 & 103 & .711 & .07 \\
\hline Trauma Type & -.11 & .17 & -.63 & 103 & .533 & -.12 \\
\hline QRI-Depth Trajectory (slope) & -.00 & .00 & -.34 & 103 & .735 & -.07 \\
\hline End-State PTSD & .00 & .00 & 2.05 & 103 & .043 & .40 \\
\hline Trauma Type & -.00 & .00 & -1.79 & 103 & .076 & -.35 \\
\hline \multicolumn{7}{|c|}{ Step 2} \\
\hline Effect & $B$ & $S E$ & $t$ & $d f$ & $p$ & $d$ \\
\hline Initial Status QRI-Depth (intercept) & 3.11 & .12 & 25.42 & 102 & $<.001$ & 5.03 \\
\hline End-State PTSD & -.01 & .00 & -1.24 & 102 & .217 & -.25 \\
\hline Trauma Type & -.09 & .16 & -.56 & 102 & .580 & -.11 \\
\hline Interaction & .01 & .01 & 2.08 & 102 & .040 & .41 \\
\hline QRI-Depth Trajectory (slope) & -.00 & .00 & -.28 & 102 & .784 & -.05 \\
\hline End-State PTSD & .00 & .00 & 1.50 & 102 & .137 & .30 \\
\hline Trauma Type & -.00 & .00 & -1.83 & 102 & .071 & -.36 \\
\hline Interaction & -.00 & .00 & -.32 & 102 & .748 & -.06 \\
\hline
\end{tabular}




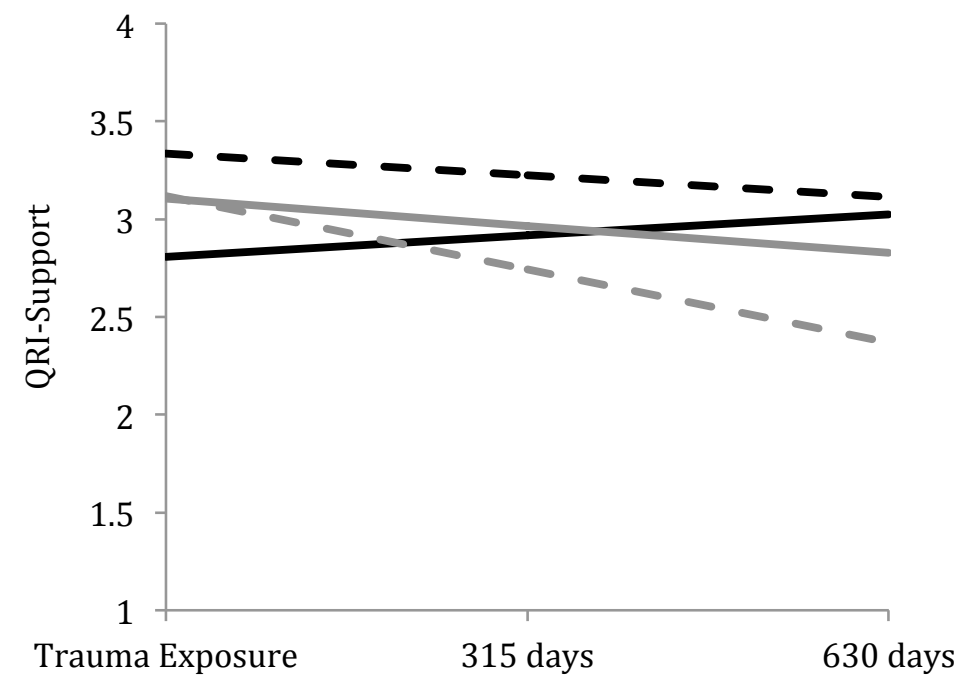

- Non Interpersonal Trauma End-State PTSD +1SD

- - Non Interpersonal Trauma End-State PTSD -1SD

Interpersonal Trauma EndState PTSD +1SD

- - Interpersonal Trauma EndState PTSD -1SD

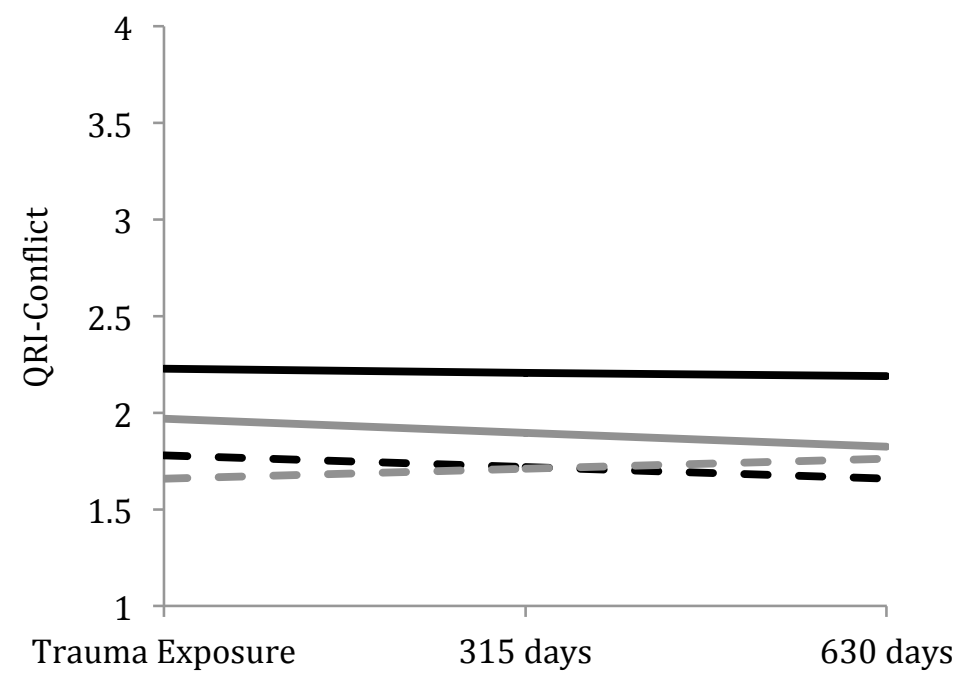

Non Interpersonal Trauma End-State PTSD +1SD

- - Non Interpersonal Trauma End-State PTSD -1SD

Interpersonal Trauma EndState PTSD +1SD

- Interpersonal Trauma EndState PTSD -1SD

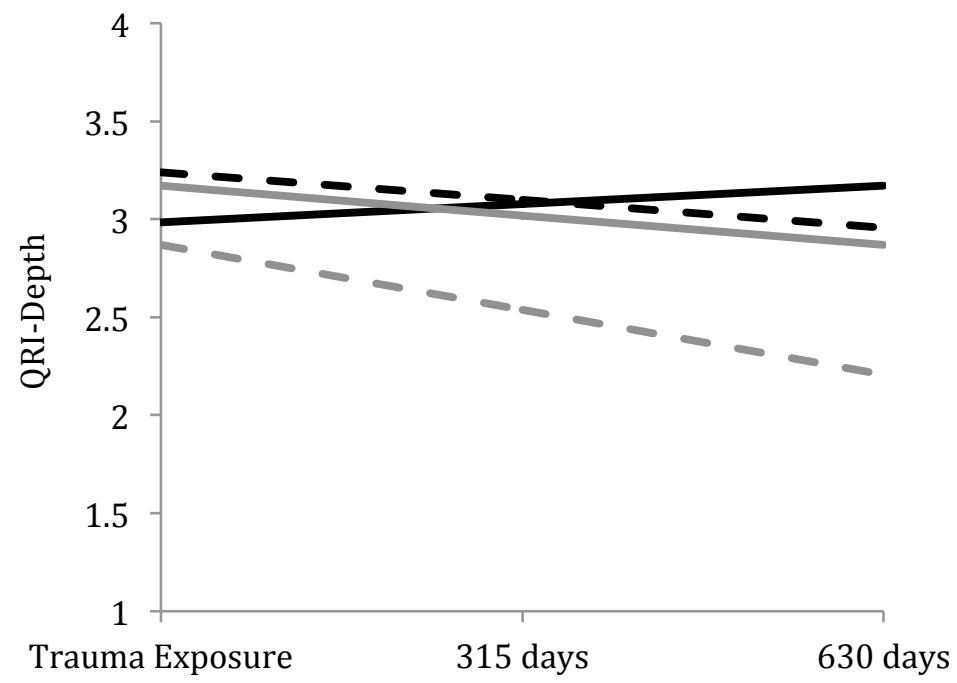

- Non Interpersonal Trauma End-State PTSD +1SD

- - Non Interpersonal Trauma End-State PTSD -1SD

- Interpersonal Trauma EndState PTSD +1SD

- Interpersonal Trauma EndState PTSD -1SD 
Figure 5. Trajectories of the QRI subscales by type of trauma exposure across high and low levels of projected end-state PTSD. Trajectories are depicted at 1 standard deviation above and below the mean of the projected end-state PTSD for interpretation.

Note. End-State PTSD = projected end-state PTSD symptom severity based on the ClinicianAdministered PTSD Scale; QRI = Quality of Relationships Inventory. 
Results of the models predicting interpersonal relationship functioning using time, projected end-state PTSD symptom severity, type of close other (intimate vs. non-intimate), and the interaction of projected end-state PTSD symptom severity by type of close other can be found in Table 7. When entered without the interaction (Step 1), similar to the findings in Aim 2, there was a significant effect of projected end-state PTSD symptom severity on the initial status (i.e., intercept) for QRI-Support and QRI-Conflict, but not QRI-Depth. Additionally, there was a marginally significant effect of close other type on the initial status of QRI-Support and a significant effect on QRI-Depth, such that those responding in reference to an intimate close other had greater initial relationship support and depth compared with those who responded in reference to a non-intimate close other. When considering the trajectory (i.e., slope) of relationship functioning over time, consistent with the findings in Aim 2, there was not a significant effect of projected end-state PTSD symptom severity on the trajectories of any of the QRI subscales. However, there was a significant effect of close other type on the trajectory of QRI-Support and -Depth, and a marginally significant effect on QRI-Conflict, such that the course of QRI-Support, Conflict, and -Depth over time was poorer for individuals who responded in reference to a non-intimate partner compared to those who responded in reference to an intimate partner.

After the interaction was added to the model (Step 2), results indicated that there was a significant end-state PTSD symptom severity by close other type interaction effect on the initial status of QRI-Conflict, but not -Support or -Depth. These findings suggest that the effect of endstate PTSD symptom severity on initial QRI-Conflict differed between those who responded in reference to intimate partners compared with those who responded in reference to non-intimate close others. Post-hoc analyses, conducted to probe and further interpret this interaction, 
suggested that there was a significant positive association between projected end-state PTSD symptom severity and initial QRI-Conflict for individuals who responded in reference to an intimate partner, $b=.02, S E=.01, t(102)=2.70, p=.008, d=.53$, but not those responded in reference to a non-intimate close other, $b=.00, S E=.00, t(102)=1.46, p=.148, d=.29$. In contrast, the effect of the end-state PTSD symptom severity by close other type interaction was not significant for the trajectory of any of the QRI subscales, suggesting that the effect of endstate PTSD symptom severity on the trajectory of interpersonal relationship functioning did not differ across types of close others (see Figure 6). 
Table 7

Growth Curve Models Predicting Interpersonal Relationship Functioning Initial Status and

Trajectory by Projected End-State PTSD Severity, Type of Close Other, and their Interaction.

\begin{tabular}{|c|c|c|c|c|c|c|}
\hline \multicolumn{7}{|c|}{ QRI-Support } \\
\hline \multicolumn{7}{|c|}{ Step 1} \\
\hline Effect & $B$ & $S E$ & $t$ & $d f$ & $p$ & $d$ \\
\hline Initial Status QRI-Support (intercept) & 3.05 & .08 & 36.19 & 103 & $<.001$ & 7.13 \\
\hline End-State PTSD & -.01 & .00 & -2.26 & 103 & .026 & -.44 \\
\hline Close Other Type & .34 & .17 & 1.98 & 103 & .051 & .39 \\
\hline QRI-Support Trajectory (slope) & -.00 & .00 & -2.44 & 103 & .017 & -.48 \\
\hline End-State PTSD & .00 & .00 & 1.21 & 103 & .230 & .24 \\
\hline Close Other Type & .00 & .00 & 2.28 & 103 & .025 & .45 \\
\hline \multicolumn{7}{|c|}{ Step 2} \\
\hline Effect & $B$ & $S E$ & $t$ & $d f$ & $p$ & $d$ \\
\hline Initial Status QRI-Support (intercept) & 3.05 & .08 & 36.21 & 102 & $<.001$ & 7.17 \\
\hline End-State PTSD & -.01 & .00 & -1.86 & 102 & .066 & -.37 \\
\hline Close Other Type & .35 & .18 & 2.02 & 102 & .046 & .40 \\
\hline Interaction & -.00 & .01 & -.37 & 102 & .710 & -.07 \\
\hline QRI-Support Trajectory (slope) & -.00 & .00 & -2.43 & 102 & .017 & -.48 \\
\hline End-State PTSD & .00 & .00 & 1.17 & 102 & .244 & .23 \\
\hline Close Other Type & .00 & .00 & 2.29 & 102 & .024 & .45 \\
\hline Interaction & -.00 & .00 & -.55 & 102 & .584 & -.11 \\
\hline
\end{tabular}




\begin{tabular}{|c|c|c|c|c|c|c|}
\hline \multicolumn{7}{|c|}{ QRI-Conflict } \\
\hline \multicolumn{7}{|c|}{ Step 1} \\
\hline Effect & $B$ & $S E$ & $t$ & $d f$ & $p$ & $d$ \\
\hline Initial Status QRI-Conflict (intercept) & 1.85 & .07 & 24.95 & 103 & $<.001$ & 4.92 \\
\hline End-State PTSD & .01 & .00 & 2.56 & 103 & .012 & .50 \\
\hline Close Other Type & .21 & .16 & 1.27 & 103 & .207 & .25 \\
\hline QRI-Conflict Trajectory (slope) & .00 & .00 & .03 & 103 & .973 & .01 \\
\hline End-State PTSD & -.00 & .00 & -.33 & 103 & .742 & -.07 \\
\hline Close Other Type & -.00 & .00 & -1.75 & 103 & .083 & -.35 \\
\hline \multicolumn{7}{|c|}{ Step 2} \\
\hline Effect & $B$ & $S E$ & $t$ & $d f$ & $p$ & $d$ \\
\hline Initial Status QRI-Conflict (intercept) & 1.84 & .07 & 25.09 & 102 & $<.001$ & 4.97 \\
\hline End-State PTSD & .00 & .00 & 1.46 & 102 & .148 & .29 \\
\hline Close Other Type & .16 & .16 & .99 & 102 & .326 & .20 \\
\hline Interaction & .02 & .01 & 2.01 & 102 & .047 & .40 \\
\hline QRI-Conflict Trajectory (slope) & .00 & .00 & .10 & 102 & .921 & .02 \\
\hline End-State PTSD & .00 & .00 & .66 & 102 & .512 & .13 \\
\hline Close Other Type & -.00 & .00 & -1.57 & 102 & .120 & -.31 \\
\hline Interaction & -.00 & .00 & -1.64 & 102 & .104 & -.32 \\
\hline
\end{tabular}




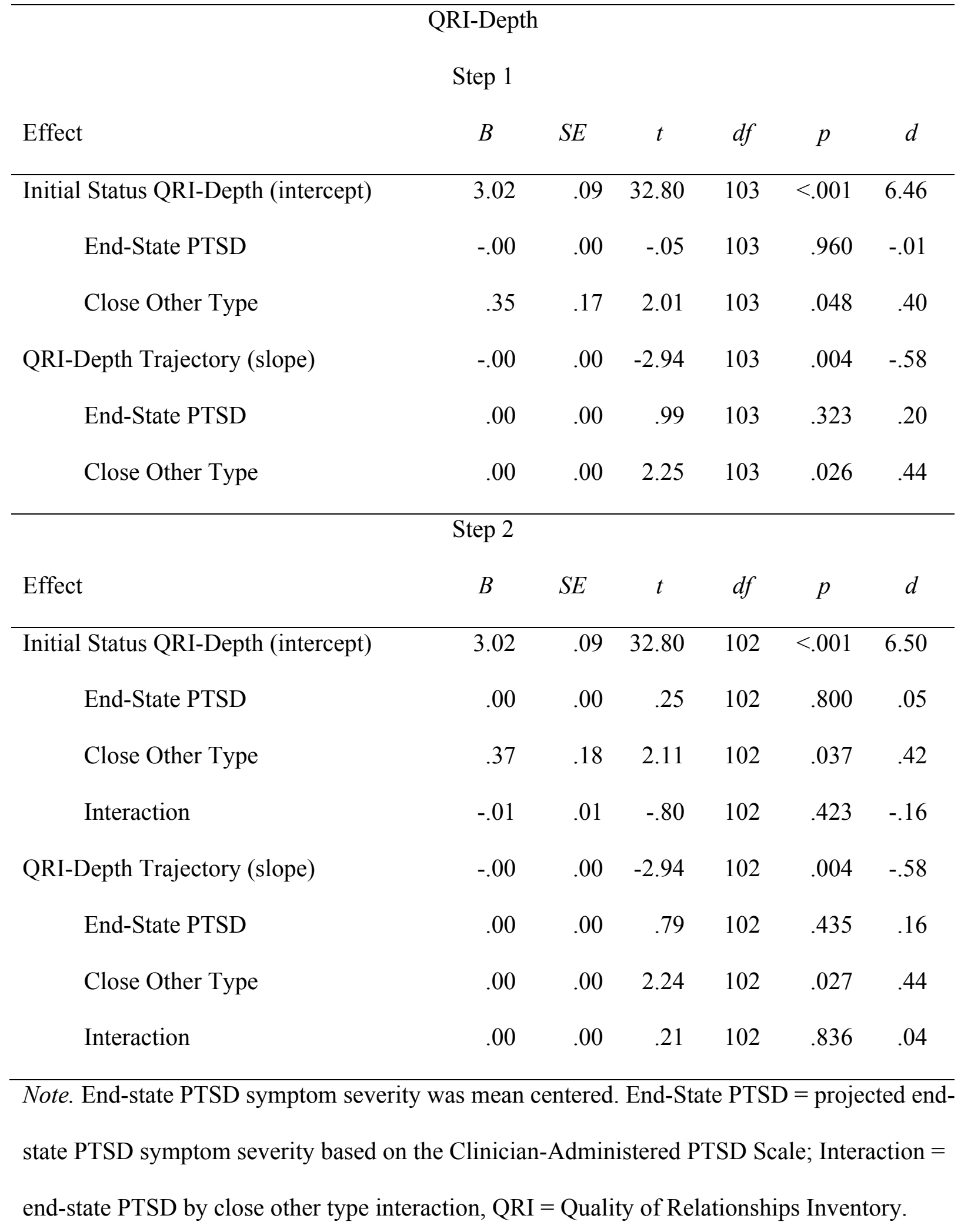



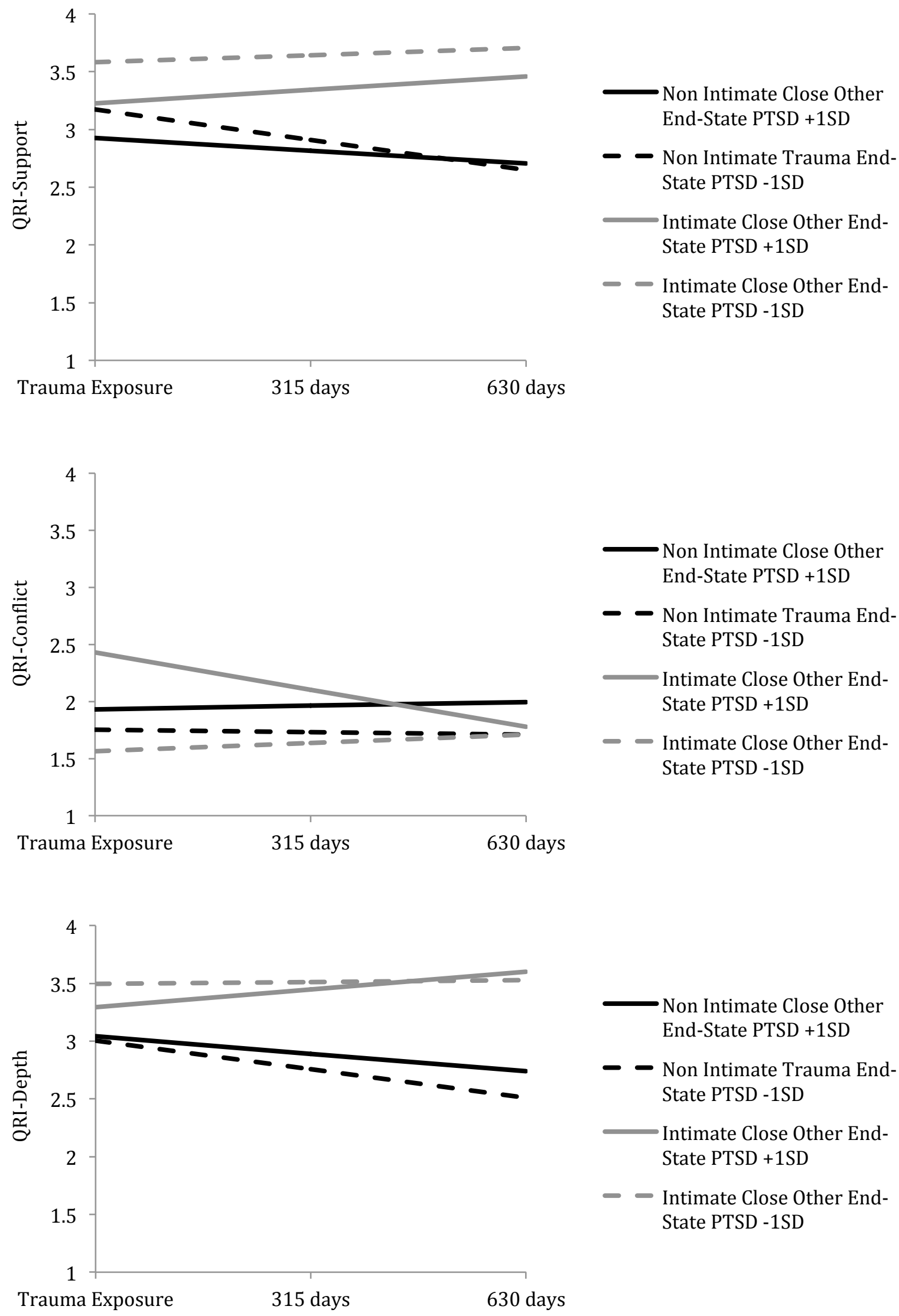
Figure 6. Trajectories of the QRI subscales by type of close other across high and low levels of projected end-state PTSD. Trajectories are depicted at 1 standard deviation above and below the mean of the projected end-state PTSD.

Note. End-State PTSD = projected end-state PTSD symptom severity based on the ClinicianAdministered PTSD Scale; QRI = Quality of Relationships Inventory. 


\begin{abstract}
$\operatorname{Aim} 4$
Table 8 contains descriptive statistics for the various time structures that were evaluated, and Table 9 contains parameter estimates and the deviance statistics from the growth curve models used to evaluate these time structures. The time structure with 6 segments yielded the smallest deviance estimate, indicating that it was the best fitting model. Accordingly, the 6-time segment structure was imposed, and attempts were made to use this time structure when constructing the LDS models. Unfortunately, likely due to the increase in missing data that resulted from sorting data points across 6 time segments (i.e., every participant had a minimum of two missing data points), the 6 time segment univariate LDS models could not converge and thus could not produce parameter and model fit estimates. Accordingly, the 5-segment time structure, which provided a similar but slightly poorer fit compared to the 6-time segment structure, was imposed when constructing the LDS models.
\end{abstract}


Table 8

Descriptive Statistics for Time Structures.

\begin{tabular}{lcccc}
\hline \multicolumn{4}{c}{ Four Time Segment (Assessment Occasion-based) Structure } \\
& Assessment 1 & Assessment 2 & Assessment 3 & Assessment 4 \\
\hline$n$ & 107 & 93 & 93 & 86 \\
$M$ Days & 124.22 & 261.05 & 386.23 & 513.80 \\
$S D$ Days & 52.78 & 57.47 & 55.93 & 56.10
\end{tabular}

Five Time Segment Structure

$\leq 120$ days $\quad 121-240$ days $\quad 241-360$ days $\quad 361-480$ days $\quad \geq 481$ days

\begin{tabular}{llcccc}
\hline$n$ & 52 & 95 & 89 & 81 & 60 \\
$M$ Days & 78.38 & 183.73 & 314.71 & 426.62 & 542.40 \\
$S D$ Days & 24.79 & 34.05 & 32.90 & 35.34 & 40.04
\end{tabular}

Six Time Segment Structure

\begin{tabular}{lcccccc} 
& $\leq 90$ & $91-180$ & $181-270$ & $271-360$ & $361-450$ & $\geq 451$ \\
& days & days & days & days & days & days \\
\hline$n$ & 32 & 63 & 65 & 75 & 67 & 73 \\
$M$ Days & 63.53 & 136.81 & 218.71 & 323.79 & 416.66 & 526.17 \\
$S D$ Days & 19.48 & 28.13 & 26.98 & 23.15 & 33.03 & 44.52 \\
\hline
\end{tabular}


Table 9

Parameter Estimates and Deviance Statistics from Growth Curve Models used to Evaluate Time Structures.

\begin{tabular}{|c|c|c|c|c|c|c|}
\hline & \multicolumn{2}{|c|}{ Four Time } & \multicolumn{2}{|c|}{ Five Time } & \multicolumn{2}{|c|}{ Six Time } \\
\hline & \multicolumn{2}{|c|}{ Segment Structure } & \multicolumn{2}{|c|}{ Segment Structure } & \multicolumn{2}{|c|}{ Segment Structure } \\
\hline & Est. & $S E$ & Est. & $S E$ & Est. & $S E$ \\
\hline Deviance & 3226.85 & & 3222.34 & & 3216.89 & \\
\hline Intercept & 50.64 & 2.91 & 54.47 & 3.18 & 54.04 & 3.12 \\
\hline Slope & -8.39 & .76 & -7.94 & .72 & -6.11 & .56 \\
\hline Intercept Variance & 699.67 & $26.45^{\mathrm{a}}$ & 803.48 & $28.35^{\mathrm{a}}$ & 739.36 & $27.19^{\mathrm{a}}$ \\
\hline Slope Variance & 29.02 & $5.39^{\mathrm{a}}$ & 26.33 & $5.13^{\mathrm{a}}$ & 14.07 & $3.75^{\mathrm{a}}$ \\
\hline Residual Variance & 124.39 & $11.15^{\mathrm{a}}$ & 122.25 & $11.06^{\mathrm{a}}$ & 132.46 & $11.51^{\mathrm{a}}$ \\
\hline \multicolumn{7}{|c|}{ sorted into their corresponding time segments and used as the outcome in growth curve } \\
\hline \multicolumn{7}{|c|}{ models in order to evaluate the time structures. The 4-time segment structure was based } \\
\hline on assessment occa & n. Est. $=$ & ramete & imate. & & & \\
\hline
\end{tabular}


Table 10 presents the model fit estimates and results of the chi-square difference tests for the six univariate models for CAPS, PCL, and QRI subscales. The univariate LDS model analyses indicated that the dual variable proportional change model was the best fit to the data for both the CAPS and PCL. In contrast, the constant change model resulted in the best fit to the data for the QRI-Support and -Depth subscales, whereas the fixed proportional change model resulted in the best fit for the QRI-Conflict subscale. Model parameter estimates for the best fitting univariate models for the CAPS and PCL can be found in Table 11 and for the QRI subscales can be found in Table 12 . 
Table 10

Model Fit Estimates and Results of the Chi-Square Difference Tests for the Univariate Latent Difference Score Models

\begin{tabular}{|c|c|c|c|c|c|c|c|}
\hline \multicolumn{8}{|c|}{ CAPS } \\
\hline Model & Parameters & $\mathrm{df}$ & $\chi^{2}(p)$ & $\operatorname{RMSEA}(p)$ & CFI & AIC & $\chi_{\text {diff }}^{2}(p)$ \\
\hline No Change & 1 & 19 & $622.32(<.001)$ & $.55(<.001)$ & .00 & 3814.87 & $<.001$ \\
\hline Constant Change & 6 & 14 & $31.79(.004)$ & $.11(.031)$ & .94 & 3234.34 & $<.001$ \\
\hline Fixed Proportional Change & 4 & 16 & $104.25(<.001)$ & $.23(<.001)$ & .69 & 3302.81 & $<.001$ \\
\hline Variable Proportional Change & 7 & 13 & $97.95(<.001)$ & $.25(<.001)$ & .70 & 3302.51 & $<.001$ \\
\hline Dual Fixed Proportional Change & 7 & 13 & $24.36(.028)$ & $.09(.113)$ & .96 & 3228.91 & .009 \\
\hline Dual Variable Proportional Change & 10 & 10 & $12.76(.238)$ & $.05(.437)$ & .99 & 3223.32 & \\
\hline \multicolumn{8}{|c|}{ PCL } \\
\hline Model & Parameters & df & $\chi^{2}(p)$ & $\operatorname{RMSEA}(p)$ & CFI & AIC & $\chi_{\text {diff }}^{2}(p)$ \\
\hline No Change & 1 & 19 & $910.77(<.001)$ & $.67(<.001)$ & .00 & 3726.35 & $<.001$ \\
\hline Constant Change & 6 & 14 & $23.65(.051)$ & $.08(.173)$ & .95 & 2849.22 & .017 \\
\hline Fixed Proportional Change & 4 & 16 & $38.82(.001)$ & $.17(.013)$ & .89 & 2860.39 & $<.001$ \\
\hline Variable Proportional Change & 7 & 13 & $31.44(.003)$ & $.17(.022)$ & .91 & 2859.01 & $<.001$ \\
\hline
\end{tabular}




$\begin{array}{lccccccc}\text { Dual Fixed Proportional Change } & 7 & 13 & 19.95(.097) & .07(.260) & .97 & 2847.52 & .039 \\ \text { Dual Variable Proportional Change } & 10 & 10 & 11.59(.314) & .04(.521) & .99 & 2845.15\end{array}$

\begin{tabular}{lccccccc}
\hline Model & \multicolumn{7}{c}{ QRI-Support } \\
& Parameters & df & $\chi^{2}(p)$ & RMSEA $(p)$ & CFI & AIC & $\chi_{\text {diff }}^{2}(p)$ \\
\hline No Change & 1 & 19 & $921.66(<.001)$ & $.67(<.001)$ & .00 & 1577.09 & $<.001$ \\
Constant Change & 6 & 14 & $16.52(.283)$ & $.04(.526)$ & .98 & 681.95 & .877 \\
Fixed Proportional Change & 4 & 16 & $30.27(.017)$ & $.09(.087)$ & .91 & 691.70 & .021 \\
Variable Proportional Change & 7 & 13 & $30.25(.004)$ & $.11(.029)$ & .89 & 697.68 & .002 \\
Dual Fixed Proportional Change & 7 & 13 & $15.51(.277)$ & $.04(.510)$ & .98 & 682.94 & .978 \\
Dual Variable Proportional Change & 10 & 10 & $15.32(.121)$ & $.07(.277)$ & .97 & 688.75 \\
\hline
\end{tabular}

QRI-Conflict

\begin{tabular}{lccccccc} 
Model & Parameters & df & $\chi^{2}(p)$ & RMSEA $(p)$ & CFI & AIC & $\chi_{\text {diff }}^{2}(p)$ \\
\hline No Change & 1 & 19 & $550.27(<.001)$ & $.51(<.001)$ & .00 & 1066.38 & $<.001$ \\
Constant Change & & & (Model covariance matrix was not positive definite) & \\
Fixed Proportional Change & 4 & 16 & $16.42(.424)$ & $.02(.680)$ & 1.00 & 538.26 & .202 \\
Variable Proportional Change & 7 & 13 & $13.47(.412)$ & $.02(.646)$ & 1.00 & 541.58 & Imp.
\end{tabular}


(Model could not converge)

\begin{tabular}{lccccccc}
\hline & \multicolumn{7}{c}{ QRI-Depth } \\
Model & Parameters & df & $\chi^{2}(p)$ & RMSEA $(p)$ & CFI & AIC & $\left.\chi_{\text {diff }}^{2} p\right)$ \\
\hline No Change & 1 & 19 & $929.84(<.001)$ & $.67(<.001)$ & .00 & 1579.61 & $<.001$ \\
Constant Change & 6 & 14 & $24.85(.036)$ & $.09(.138)$ & .94 & 684.62 & .770 \\
Fixed Proportional Change & 4 & 16 & $34.24(.005)$ & $.10(.037)$ & .91 & 690.01 & .082 \\
Variable Proportional Change & 7 & 13 & $34.04(.001)$ & $.12(.011)$ & .89 & 695.81 & .012 \\
Dual Fixed Proportional Change & 7 & 13 & $23.43(.037)$ & $.09(.135)$ & .95 & 685.20 & .942 \\
Dual Variable Proportional Change & 10 & 10 & $23.04(.011)$ & $.11(.048)$ & .93 & 690.80
\end{tabular}

Note. Chi-square difference tests were conducted by comparing each model to the dual variable proportional change model. For the QRI-conflict subscale, the dual variable proportional change model could not converge; accordingly, subsequent models were compared to the dual fixed proportional change models. When multiple models resulted in a better fit to the data than the dual variable proportional change model, model fit was further evaluated by comparing those models using the chi-square difference test and selecting the more parsimonious model. CAPS = Clinician-Administered PTSD Scale; PCL = PTSD Checklist; QRI = Quality of Relationships Inventory; Imp. = impossible value. 
Table 11

Model Parameter Estimates for the Best Fitting PTSD Univariate Latent Difference Score Models

\begin{tabular}{|c|c|c|c|c|c|c|}
\hline \multirow[b]{2}{*}{ Parameters } & \multicolumn{3}{|c|}{ CAPS Dual Variable Proportional Change } & \multicolumn{3}{|c|}{ PCL Dual Variable Proportional Change } \\
\hline & Est. & $S E$ & $p$ & Est. & $S E$ & $p$ \\
\hline \multicolumn{7}{|c|}{ Constant Coefficients } \\
\hline$x_{0}$ & 46.00 & 3.10 & $<.001$ & 42.02 & 1.83 & $<.001$ \\
\hline$x_{s}$ & 5.89 & 3.36 & .079 & 11.61 & 6.02 & .054 \\
\hline$x_{0} x_{S}$ & 137.64 & 52.10 & .008 & 42.88 & 23.61 & .069 \\
\hline \multicolumn{7}{|c|}{ Proportional Coefficients } \\
\hline$\beta_{1}$ & -.28 & .08 & .001 & -.33 & .14 & .021 \\
\hline$\beta_{2}$ & -.47 & .09 & $<.001$ & -.47 & .16 & .002 \\
\hline$\beta_{3}$ & -.41 & .12 & .001 & -.43 & .18 & .021 \\
\hline$\beta_{4}$ & -.54 & .13 & $<.001$ & -.47 & .20 & .017 \\
\hline \multicolumn{7}{|c|}{ Error Variances } \\
\hline$e_{[1-5]}$ & 108.29 & 11.79 & $<.001$ & 57.30 & 6.22 & $<.001$ \\
\hline
\end{tabular}


Note: Est. $=$ parameter estimate $x_{0}=$ estimated mean for initial status; $x_{\mathrm{s}}=$ estimated mean for the non-stationarity $/$ constant change effect; $\beta_{1-4}=$ autoregressive/proportional change effects; $e_{[1-5]}=$ error variances; $\mathrm{CAPS}=$ Clinician-Administered PTSD Scale; PCL = PTSD Checklist. Error terms were constrained to be equal. 
Table 12

Model Parameter Estimates for the Best Fitting Interpersonal Relationship Functioning Univariate Latent Difference Score Models

\begin{tabular}{|c|c|c|c|c|c|c|c|c|}
\hline \multirow[b]{3}{*}{ Parameters } & \multicolumn{3}{|c|}{ QRI-Support } & \multicolumn{2}{|c|}{ QRI-Conflict } & \multicolumn{3}{|c|}{ QRI-Depth } \\
\hline & \multicolumn{3}{|c|}{ Constant Change } & \multicolumn{2}{|c|}{ Fixed Proportional Change } & \multicolumn{3}{|c|}{ Constant Change } \\
\hline & Est. & $S E$ & $p$ & Est. & $p$ & Est. & $S E$ & $p$ \\
\hline Constant Coefficients & 2.09 & .07 & $<.001$ & & & 2.07 & .08 & $<.001$ \\
\hline$x_{0}$ & -.04 & .03 & .087 & & & -.05 & .02 & .041 \\
\hline$x_{s}$ & -.00 & .02 & .952 & & & -.01 & .02 & .801 \\
\hline
\end{tabular}

Proportional Coefficient

$\beta$

$-.01 \quad .02 \quad .462$

Error Variances

$\begin{array}{lllllllllll}e_{[1-5]} & .20 & .02 & <.001 & .15 & .02 & <.001 & .18 & .02 & <.001\end{array}$

Note: Est. $=$ parameter estimate; $x_{0}=$ estimated mean for initial status; $x_{\mathrm{s}}=$ estimated mean for the non-stationarity/constant change effect; $\beta=$ autoregressive/proportional change effect; $e_{[1-5]}=$ error variances; QRI = Quality of Relationships Inventory. Error terms were constrained to be equal. 
The pairing of the best fitting univariate LDS models for PTSD with the best fitting LDS models for interpersonal relationship functioning resulted in the following six bivariate LDS models: 1) CAPS dual variable proportional change with QRI-Support constant change, 2) CAPS $_{\text {dual variable proportional }}$ change with QRI-Conflict $f_{\text {fixed proportional change, }}$ 3) CAPS $_{\text {dual variable proportional change }}$ with QRI-Depth ${ }_{\text {constant }}$

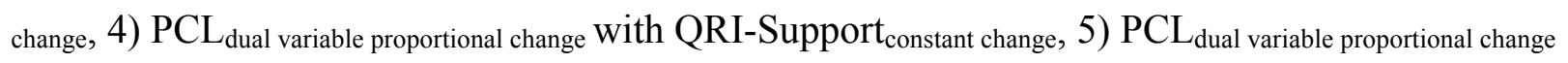
with QRI-Conflict fixed proportional change, 6) PCL $\mathrm{L}_{\text {dual variable proportional change }}$ with $\mathrm{QRI}$-Depth constant change. $_{\text {. }}$ Figure 7 presents the path diagram for the bivariate LDS models for dual variable proportional change for the PTSD variable and constant change for the interpersonal relationship functioning variable (models 1, 3, 4, and 6 above). Figure 8 presents the path diagram for the bivariate LDS models for dual variable proportional change for the PTSD variable and fixed proportional change for the interpersonal relationship functioning variable (models 2 and 5 above). Tables 1318 contain the model fit indices and parameter estimates for the bivariate LDS models. Notably, none of the cross-lagged effects were significant in any of the bivariate LDS models. 


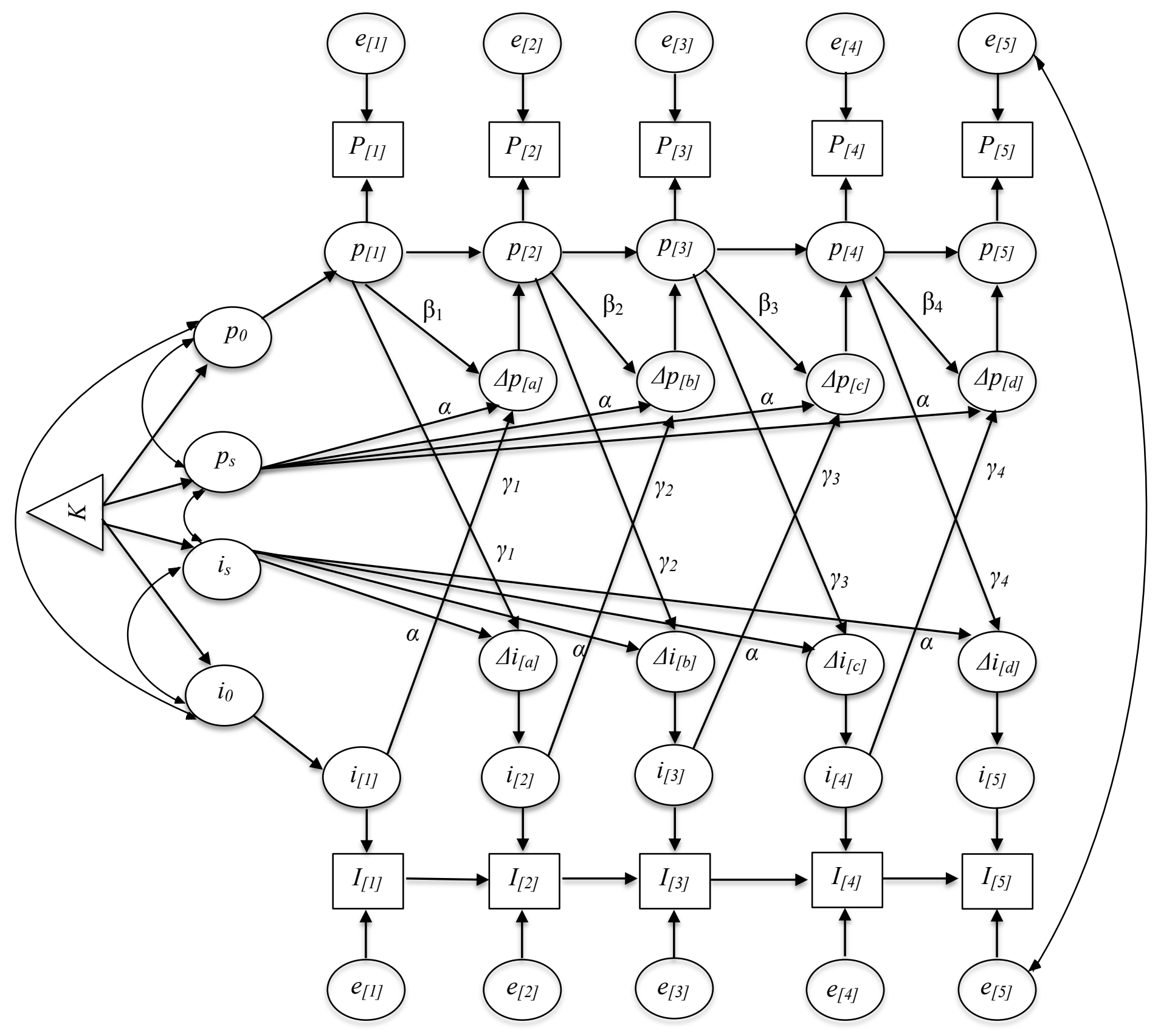

Figure 7. Path diagram for the bivariate latent difference score (LDS) model for dual variable proportional change for PTSD and constant change for interpersonal relationship functioning. This model was utilized for bivariate LDS models examining the CAPS with the QRI-Support, the PCL with the QRI-Support, the CAPS with the QRI-Depth, and the PCL with the QRI-Depth. 
Squares represent observed variables, circles represent latent (or unobserved) variables, and the triangle $/ K$ represents a constant used to estimate means and intercepts. Single-headed arrows indicate a regression coefficient, intercept, or mean. Double-headed arrows indicate a covariance. $P_{[t]}$ represents the observed PTSD variable score at time $t, p_{[t]}$ represents the latent PTSD variable score at time $t$, and $\Delta p_{[t]}$ represents the PTSD LDS between corresponding time points. $p_{0}$ represents the estimated mean for the initial status for the PTSD variable and $\alpha \times p_{\mathrm{s}}$ represents the non-stationarity/constant change for the PTSD variable. $I_{[t]}$ represents the observed interpersonal relationship functioning variable score at time $t, i_{[t]}$ represents the latent interpersonal relationship functioning variable score at time $t$, and $\Delta i_{[t]}$ represents the interpersonal relationship functioning LDS between corresponding time points. $i_{0}$ represents the estimated mean for the initial status for the interpersonal relationship functioning variable and $\alpha$ $\times i_{\mathrm{S}}$ represents the non-stationarity/constant change for the interpersonal relationship functioning variable. $\beta_{\mathrm{t}}$ represents the autoregressive/proportional change for the PTSD as a function of the latent PTSD variable at time $t$ on the subsequent LDS for PTSD and $\gamma_{t}$ represent the cross-lagged effects from one variable at time $t$ on the subsequent LDS for the other variable. $e_{[t]}$ represents the error term at time $t$. Error terms were constrained to be equal within variables.

Note. CAPS $=$ Clinician-Administered PTSD Scale; PCL = PTSD Checklist QRI = Quality of Relationships Inventory. 


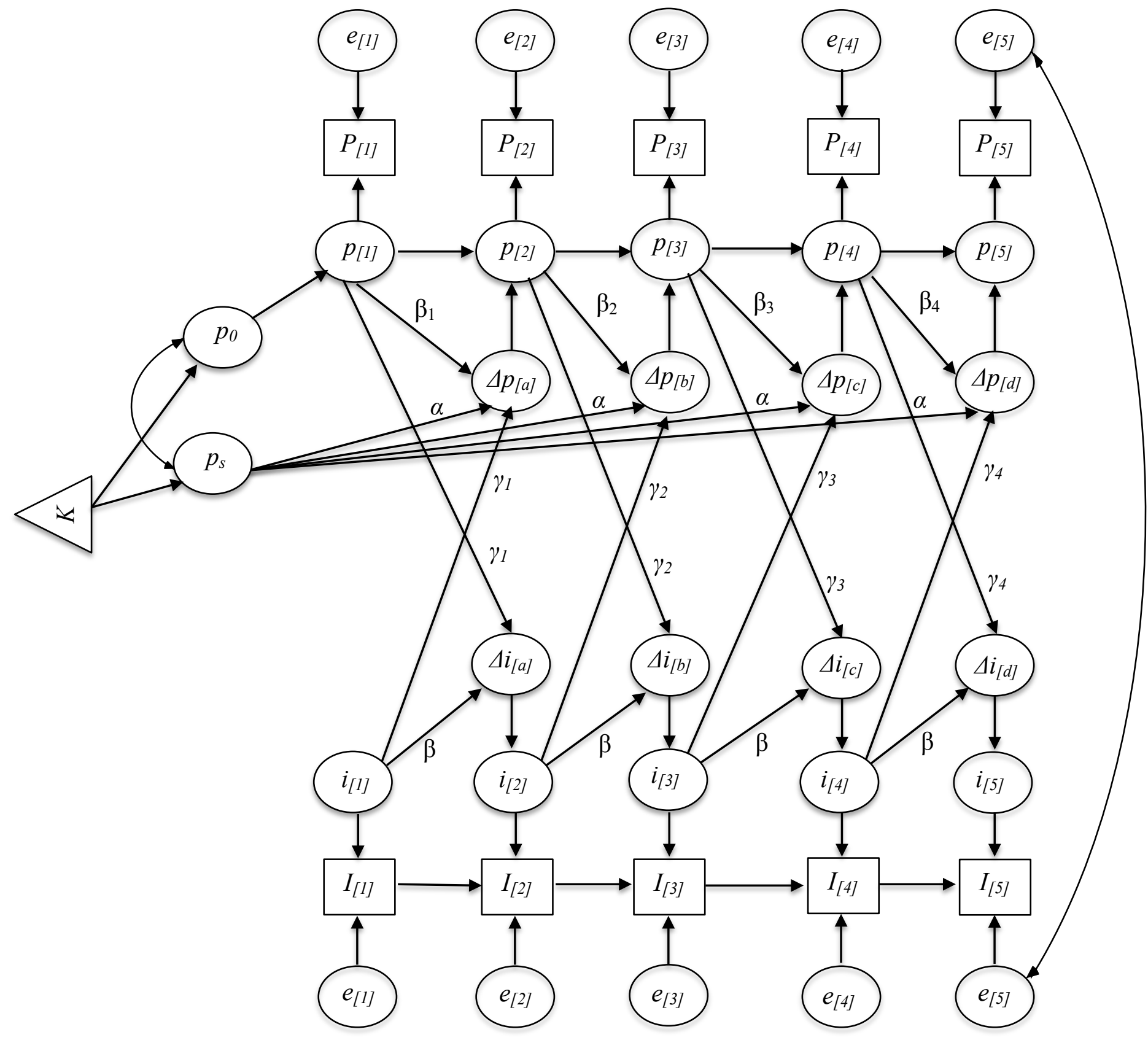


Figure 8. Path diagram for the bivariate latent difference score (LDS) model for dual variable proportional change for PTSD and fixed proportional change for interpersonal relationship functioning. This model was utilized for bivariate LDS models examining the CAPS with the QRI-Conflict and the PCL with the QRI-Conflict. Squares represent observed variables, circles represent latent (or unobserved) variables, and the triangle $/ K$ represents a constant used to estimate means and intercepts. Single-headed arrows indicate a regression coefficient, intercept, or mean. Double-headed arrows indicate a covariance. $P_{[t]}$ represents the observed PTSD variable score at time $t, p_{[t]}$ represents the latent PTSD variable score at time $t$, and $\Delta p_{[t]}$ represents the PTSD LDS between corresponding time points. $p_{0}$ represents the estimated mean for the initial status for the PTSD variable and $\alpha \times p_{\text {s }}$ represents the non-stationarity/constant change for the PTSD variable. $I_{[t]}$ represents the observed interpersonal relationship functioning variable score at time $t, i_{[t]}$ represents the latent interpersonal relationship functioning variable score at time $t$, and $\Delta i_{[t]}$ represents the interpersonal relationship functioning LDS between corresponding time points. $\beta_{\mathrm{t}}$ represents the autoregressive/proportional change for the PTSD as a function of the latent PTSD variable at time $t$ on the subsequent LDS for PTSD. $\beta$ represents the autoregressive/proportional change for the interpersonal relationship functioning as a function of the latent interpersonal relationship functioning variable at time $t$ on the subsequent LDS for interpersonal relationship functioning. Autoregressive/proportional change effects were held constant for interpersonal relationship functioning. $\gamma_{t}$ represent the cross-lagged effects from one variable at time $t$ on the subsequent LDS for the other variable. $e_{[t]}$ represents the error term at time $t$. Error terms were constrained to be equal within variables.

Note. CAPS = Clinician-Administered PTSD Scale; PCL = PTSD Checklist; QRI = Quality of Relationships Inventory. 
Table 13

Model Fit indices and Parameter Estimates for the CAPS with QRI-Support Bivariate Latent Difference Score Model

\begin{tabular}{|c|c|c|c|c|c|c|}
\hline \multirow[b]{2}{*}{ Parameters } & \multicolumn{3}{|c|}{ CAPS Dual Variable Proportional Change } & \multicolumn{3}{|c|}{ QRI-Support Constant Change } \\
\hline & Est. & $S E$ & $p$ & Est. & $S E$ & $p$ \\
\hline \multicolumn{7}{|c|}{ Constant Coefficients } \\
\hline$x_{0}$ & 45.78 & 3.17 & $<.001$ & 2.05 & .09 & $<.001$ \\
\hline$x_{s}$ & 16.55 & 7.70 & .032 & -.03 & .05 & .603 \\
\hline$x_{0} x_{s}$ & 149.00 & 60.67 & .014 & .01 & .02 & .830 \\
\hline$p_{0} i_{0}$ & \multicolumn{6}{|c|}{ Est. $=-2.94, S E=2.20, p=.181$} \\
\hline$p_{s} i_{s}$ & \multicolumn{6}{|c|}{ Est. $=.66, S E=.45, p=.140$} \\
\hline \multicolumn{7}{|c|}{ Proportional Coefficients } \\
\hline$\beta_{1}$ & -.31 & .17 & .007 & & & \\
\hline$\beta_{2}$ & -.55 & .12 & $<.001$ & & & \\
\hline$\beta_{3}$ & -.45 & .14 & .001 & & & \\
\hline$\beta_{4}$ & -.58 & .13 & $<.001$ & & & \\
\hline
\end{tabular}

Cross-lag Coefficients 


$\begin{array}{lllllll}\gamma_{1} & -4.45 & 3.57 & .212 & .00 & .00 & .539 \\ \gamma_{2} & -3.35 & 3.22 & .298 & -.00 & .00 & .283 \\ \gamma_{3} & -4.95 & 3.31 & .135 & .00 & .00 & .878 \\ \gamma_{4} & -5.01 & 3.38 & .139 & .00 & .00 & .856\end{array}$

Error Variances

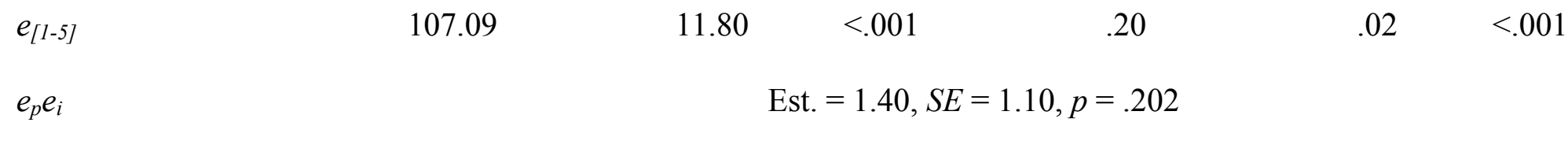

Fit indices

Parameters

df

$\chi^{2}(p)$

$\operatorname{RMSEA}(p)$

CFI

27

38

$50.22(.089)$

$.06(.397)$

.97

Note: Est. $=$ parameter estimate $; x_{0}=$ estimated mean for initial status; $x_{\mathrm{s}}=$ estimated mean for the non-stationarity/constant change effect; $x_{0} x_{\mathrm{s}}=$ covariance between the estimated mean for initial status and the estimated mean for the non-stationarity/constant change effect; $p_{0} i_{0}=$ covariance between the estimated mean for initial status on the CAPS and the QRI-Support; $p_{\mathrm{s}} i_{\mathrm{s}}=$ covariance between the estimated mean for the non-stationarity/constant change effect for the CAPS and the QRI-Support; $\beta_{1-4}=$ 
autoregressive/proportional change effects; $\gamma_{1-4}=$ cross-lagged effects; $e_{[1-5]}=$ error variances; $e_{p} e_{i}=$ covariance between error variance for the CAPS and QRI-Support; CAPS = Clinician-Administered PTSD Scale; QRI = Quality of Relationships Inventory. Error terms were constrained to be equal within variables. 
Table 14

Model Fit indices and Parameter Estimates for the PCL with QRI-Support Bivariate Latent Difference Score Model

\begin{tabular}{|c|c|c|c|c|c|c|}
\hline \multirow[b]{2}{*}{ Parameters } & \multicolumn{3}{|c|}{ PCL Dual Variable Proportional Change } & \multicolumn{3}{|c|}{ QRI-Support Constant Change } \\
\hline & Est. & $S E$ & $p$ & Est. & $S E$ & $p$ \\
\hline \multicolumn{7}{|c|}{ Constant Coefficients } \\
\hline$x_{0}$ & 42.09 & 1.89 & $<.001$ & 2.07 & .09 & $<.001$ \\
\hline$x_{s}$ & 20.68 & 8.27 & .012 & -.06 & .11 & .604 \\
\hline$x_{0} x_{s}$ & 51.12 & 26.02 & .049 & .01 & .02 & .788 \\
\hline$p_{0} i_{0}$ & \multicolumn{6}{|c|}{ Est. $=-0.66, S E=1.21, p=.583$} \\
\hline$p_{s} i_{s}$ & \multicolumn{6}{|c|}{ Est. $=.36, S E=.27, p=.193$} \\
\hline \multicolumn{7}{|c|}{ Proportional Coefficients } \\
\hline$\beta_{1}$ & -.38 & .17 & .027 & & & \\
\hline$\beta_{2}$ & -.57 & .17 & .001 & & & \\
\hline$\beta_{3}$ & -.47 & .21 & .022 & & & \\
\hline$\beta_{4}$ & -.52 & .20 & .009 & & & \\
\hline
\end{tabular}

Cross-lag Coefficients 


$\begin{array}{lllllll}\gamma_{1} & -3.30 & 2.45 & .178 & .00 & .00 & .689 \\ \gamma_{2} & -2.25 & 2.11 & .286 & -.00 & .00 & .816 \\ \gamma_{3} & -3.94 & 2.16 & .067 & .00 & .00 & .846 \\ \gamma_{4} & -3.70 & 2.30 & .107 & .00 & .00 & .833\end{array}$

Error Variances

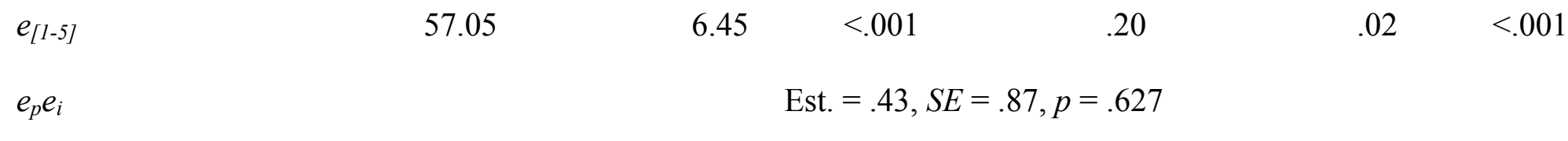

Fit indices

Parameters

df

$\chi^{2}(p)$

$\operatorname{RMSEA}(p)$

CFI

27

38

$90.11(<.001)$

$.11(.001)$

.87

Note: Est. $=$ parameter estimate $; x_{0}=$ estimated mean for initial status; $x_{\mathrm{s}}=$ estimated mean for the non-stationarity/constant change effect; $x_{0} x_{\mathrm{s}}=$ covariance between the estimated mean for initial status and the estimated mean for the non-stationarity/constant change effect; $p_{0} i_{0}=$ covariance between the estimated mean for initial status on the PCL and the QRI-Support; $p_{\mathrm{s}} i_{\mathrm{s}}=$ covariance between the estimated mean for the non-stationarity/constant change effect for the PCL and the QRI-Support; $\beta_{1-4}=$ autoregressive/proportional 
change effects; $\gamma_{1^{-} 4}=$ cross-lagged effects; $e_{[1-5]}=$ error variances; $e_{p} e_{i}=$ covariance between error variance for the PCL and QRI-

Support; PCL = PTSD Checklist; QRI = Quality of Relationships Inventory. Error terms were constrained to be equal within variables. 
Table 15

Model Fit indices and Parameter Estimates for the CAPS with QRI-Conflict Bivariate Latent Difference Score Model

\begin{tabular}{|c|c|c|c|c|c|c|}
\hline \multirow[b]{2}{*}{ Parameters } & \multicolumn{3}{|c|}{ CAPS Dual Variable Proportional Change } & \multicolumn{3}{|c|}{ QRI-Conflict Proportional Change } \\
\hline & Est. & $S E$ & $p$ & Est. & $S E$ & $p$ \\
\hline \multicolumn{7}{|c|}{ Constant Coefficients } \\
\hline$x_{0}$ & 46.08 & 3.09 & $<.001$ & & & \\
\hline$x_{s}$ & 3.82 & 3.80 & .315 & & & \\
\hline$x_{0} x_{s}$ & 141.74 & 54.52 & .009 & & & \\
\hline \multicolumn{7}{|c|}{ Proportional Coefficients } \\
\hline$\beta_{1}$ & -.28 & .11 & .009 & -.03 & .03 & .303 \\
\hline$\beta_{2}$ & -.55 & .12 & $<.001$ & -.03 & .03 & .303 \\
\hline$\beta_{3}$ & -.45 & .14 & .001 & -.03 & .03 & .303 \\
\hline$\beta_{4}$ & -.58 & .13 & $<.001$ & -.03 & .03 & .303 \\
\hline \multicolumn{7}{|c|}{ Cross-lag Coefficients } \\
\hline$\gamma_{1}$ & 2.26 & 4.58 & .623 & .00 & .00 & .821 \\
\hline$\gamma_{2}$ & 5.29 & 3.55 & .136 & .00 & .00 & .879 \\
\hline
\end{tabular}




$\begin{array}{lllllll}\gamma_{3} & 4.23 & 3.52 & .230 & .00 & .00 & .554 \\ \gamma_{4} & 2.40 & 3.78 & .526 & .00 & .00 & .658\end{array}$

Error Variances

$\begin{array}{llcccc}e_{[1-5]} & 106.45 & 11.65 & <.001 & .15 & .01\end{array}$

Fit indices

Parameters

df

$\chi^{2}(p)$

RMSEA $(p)$

CFI

23

42

$73.85(.002)$

$.08(.045)$

.93

Note: Est. $=$ parameter estimate $; x_{0}=$ estimated mean for initial status; $x_{\mathrm{s}}=$ estimated mean for the non-stationarity $/$ constant change effect; $x_{0} x_{\mathrm{s}}=$ covariance between the estimated mean for initial status and the estimated mean for the non-stationarity/constant change effect; $\beta_{1-4}=$ autoregressive/proportional change effects; $\gamma_{1-4}=$ cross-lagged effects; $e_{[1-5]}=$ error variances; $e_{p} e_{i}=$ covariance between error variance for the CAPS and QRI-Conflict; CAPS = Clinician-Administered PTSD Scale; QRI = Quality of Relationships Inventory. Error terms were constrained to be equal within variables. 
Table 16

Model Fit indices and Parameter Estimates for the PCL with QRI-Conflict Bivariate Latent Difference Score Model

\begin{tabular}{|c|c|c|c|c|c|c|}
\hline \multirow[b]{2}{*}{ Parameters } & \multicolumn{3}{|c|}{ PCL Dual Variable Proportional Change } & \multicolumn{3}{|c|}{ QRI-Conflict Proportional Change } \\
\hline & Est. & $S E$ & $p$ & Est. & $S E$ & $p$ \\
\hline \multicolumn{7}{|c|}{ Constant Coefficients } \\
\hline$x_{0}$ & 41.98 & 1.86 & $<.001$ & & & \\
\hline$x_{s}$ & 11.46 & 5.62 & .041 & & & \\
\hline$x_{0} x_{s}$ & 43.88 & 22.58 & .052 & & & \\
\hline \multicolumn{7}{|c|}{ Proportional Coefficients } \\
\hline$\beta_{1}$ & -.37 & .15 & .013 & -.02 & .04 & .600 \\
\hline$\beta_{2}$ & -.49 & .16 & .002 & -.02 & .04 & .600 \\
\hline$\beta_{3}$ & -.45 & .19 & .018 & -.02 & .04 & .600 \\
\hline$\beta_{4}$ & -.50 & .20 & .101 & -.02 & .04 & .600 \\
\hline \multicolumn{7}{|c|}{ Cross-lag Coefficients } \\
\hline$\gamma_{1}$ & 2.07 & 2.84 & .466 & -.00 & .00 & .513 \\
\hline$\gamma_{2}$ & 1.40 & 2.51 & .578 & .00 & .00 & .813 \\
\hline
\end{tabular}




$\begin{array}{lrrrrrr}\gamma_{3} & .97 & 2.62 & .712 & .00 & .00 & .875 \\ \gamma_{4} & 1.52 & 3.12 & .625 & .00 & .00 & .474\end{array}$

Error Variances
$e_{[1-5]}$
57.59
6.27
$<.001$
.15
$.01<.001$
$e_{p} e_{i}$
Est. $=.97, S E=.66, p=.143$

\section{Fit indices}

\section{Parameters}

df

$\chi^{2}(p)$

RMSEA $(p)$

CFI

23

42

$92.35(<.001)$

$.11(.002)$

.87

Note: Est. $=$ parameter estimate $; x_{0}=$ estimated mean for initial status; $x_{\mathrm{s}}=$ estimated mean for the non-stationarity $/$ constant change effect; $x_{0} x_{\mathrm{s}}=$ covariance between the estimated mean for initial status and the estimated mean for the non-stationarity/constant change effect; $\beta_{1-4}=$ autoregressive/proportional change effects; $\gamma_{1-4}=$ cross-lagged effects; $e_{[1-5]}=$ error variances; $e_{p} e_{i}=$ covariance between error variance for the PCL and QRI-Conflict; PCL = PTSD Checklist; QRI = Quality of Relationships Inventory. Error terms were constrained to be equal within variables. 
Table 17

Model Fit indices and Parameter Estimates for the CAPS with QRI-Depth Bivariate Latent Difference Score Model

\begin{tabular}{|c|c|c|c|c|c|c|}
\hline \multirow[b]{2}{*}{ Parameters } & \multicolumn{3}{|c|}{ CAPS Dual Variable Proportional Change } & \multicolumn{3}{|c|}{ QRI-Depth Constant Change } \\
\hline & Est. & $S E$ & $p$ & Est. & $S E$ & $p$ \\
\hline \multicolumn{7}{|c|}{ Constant Coefficients } \\
\hline$x_{0}$ & 45.94 & 3.18 & $<.001$ & 2.02 & .09 & $<.001$ \\
\hline$x_{s}$ & 6.72 & 5.95 & .259 & -.02 & .05 & .610 \\
\hline$x_{0} x_{s}$ & 166.37 & 64.40 & .010 & .00 & .02 & .967 \\
\hline$p_{0} i_{0}$ & \multicolumn{6}{|c|}{ Est. $=-.41, S E=2.34, p=.860$} \\
\hline$p_{s} i_{s}$ & \multicolumn{6}{|c|}{ Est. $=.61, S E=.41, p=.130$} \\
\hline \multicolumn{7}{|c|}{ Proportional Coefficients } \\
\hline$\beta_{1}$ & -.33 & .12 & .006 & & & \\
\hline$\beta_{2}$ & -.55 & .13 & $<.001$ & & & \\
\hline$\beta_{3}$ & -.47 & .14 & .001 & & & \\
\hline$\beta_{4}$ & -.58 & .14 & $<.001$ & & & \\
\hline
\end{tabular}

Cross-lag Coefficients 


$\begin{array}{lrrrrrr}\gamma_{1} & .66 & 3.24 & .838 & .00 & .00 & .530 \\ \gamma_{2} & 1.31 & 2.90 & .651 & -.00 & .00 & .213 \\ \gamma_{3} & .39 & 2.83 & .890 & .00 & .00 & .937 \\ \gamma_{4} & -.25 & 2.82 & .929 & -.00 & .00 & .641\end{array}$

Error Variances

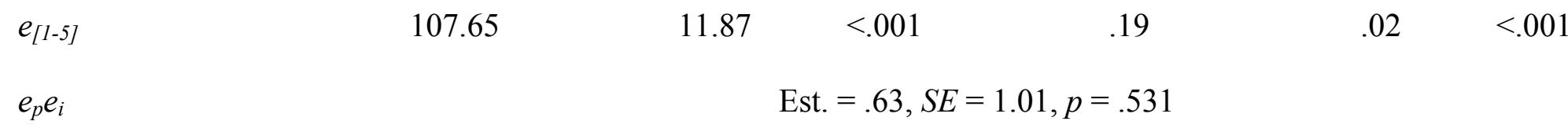

Fit indices

Parameters

df

$\chi^{2}(p)$

RMSEA $(p)$

CFI

27

$$
38
$$

$56.06(.030)$

$.07(.222)$

.96

Note: Est. $=$ parameter estimate; $x_{0}=$ estimated mean for initial status; $x_{\mathrm{s}}=$ estimated mean for the non-stationarity/constant change effect; $x_{0} x_{\mathrm{s}}=$ covariance between the estimated mean for initial status and the estimated mean for the non-stationarity/constant change effect; $p_{0} i_{0}=$ covariance between the estimated mean for initial status on the CAPS and the QRI-Depth; $p_{\mathrm{s}} i_{\mathrm{s}}=$ covariance between the estimated mean for the non-stationarity/constant change effect for the CAPS and the QRI-Depth; $\beta_{1-4}=$ autoregressive/proportional 
change effects; $\gamma_{1^{-4}}=$ cross-lagged effects; $e_{[1-5]}=$ error variances; $e_{p} e_{i}=$ covariance between error variance for the CAPS and QRIDepth; CAPS = Clinician-Administered PTSD Scale; QRI = Quality of Relationships Inventory. Error terms were constrained to be equal within variables. 
Table 18

Model Fit indices and Parameter Estimates for the PCL with QRI-Depth Bivariate Latent Difference Score Model

\begin{tabular}{|c|c|c|c|c|c|c|}
\hline \multirow[b]{2}{*}{ Parameters } & \multicolumn{3}{|c|}{ PCL Dual Variable Proportional Change } & \multicolumn{3}{|c|}{ QRI-Depth Constant Change } \\
\hline & Est. & $S E$ & $p$ & Est. & $S E$ & $p$ \\
\hline \multicolumn{7}{|c|}{ Constant Coefficients } \\
\hline$x_{0}$ & 42.35 & 1.89 & $<.001$ & 2.04 & .09 & $<.001$ \\
\hline$x_{s}$ & 13.96 & 6.53 & .033 & -.05 & .10 & .607 \\
\hline$x_{0} x_{s}$ & 44.73 & 26.25 & .088 & .00 & .02 & .998 \\
\hline$p_{0} i_{0}$ & \multicolumn{6}{|c|}{ Est. $=1.30, S E=1.32, p=.322$} \\
\hline$p_{s} i_{s}$ & \multicolumn{6}{|c|}{ Est. $=.32, S E=.23, p=.154$} \\
\hline \multicolumn{7}{|c|}{ Proportional Coefficients } \\
\hline$\beta_{1}$ & -.33 & .18 & .063 & & & \\
\hline$\beta_{2}$ & -.51 & .18 & .005 & & & \\
\hline$\beta_{3}$ & -.41 & .21 & .046 & & & \\
\hline$\beta_{4}$ & -.45 & .20 & .023 & & & \\
\hline
\end{tabular}

Cross-lag Coefficients 


$\begin{array}{lrrrrrr}\gamma_{1} & -1.18 & 2.26 & .602 & .00 & .00 & .714 \\ \gamma_{2} & -.32 & 1.78 & .857 & -.00 & .00 & .735 \\ \gamma_{3} & -1.52 & 1.78 & .393 & .00 & .00 & .704 \\ \gamma_{4} & -1.59 & 1.85 & .391 & -.00 & .00 & .762\end{array}$

Error Variances

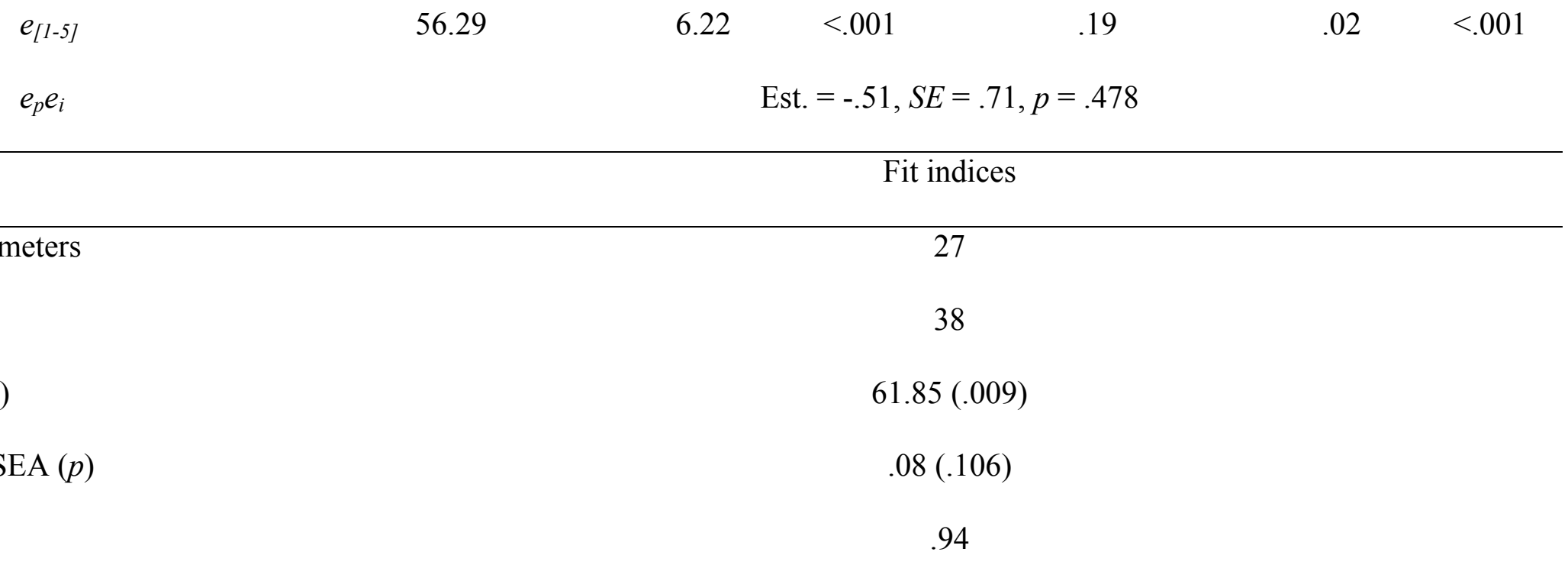

Note: Est. $=$ parameter estimate $; x_{0}=$ estimated mean for initial status; $x_{\mathrm{s}}=$ estimated mean for the non-stationarity $/$ constant change effect; $x_{0} x_{\mathrm{s}}=$ covariance between the estimated mean for initial status and the estimated mean for the non-stationarity/constant change effect; $p_{0} i_{0}=$ covariance between the estimated mean for initial status on the PCL and the QRI-Depth; $p_{\mathrm{s}} i_{\mathrm{s}}=$ covariance between the estimated mean for the non-stationarity/constant change effect for the PCL and the QRI-Depth; $\beta_{1-4}=$ autoregressive/proportional 
change effects; $\gamma_{1^{-} 4}=$ cross-lagged effects; $e_{[1-5]}=$ error variances; $e_{p} e_{i}=$ covariance between error variance for the PCL and QRIDepth; PCL = PTSD Checklist; QRI = Quality of Relationships Inventory. Error terms were constrained to be equal within variables. 


\section{Discussion}

The current study sought to examine the trajectory of posttraumatic symptomatology and interpersonal relationship functioning, as well as the effects that these constructs exert on one another over time, in a community sample of individuals who were recently exposed to traumatic events. Overall, PTSD symptom severity was found to decline over time following trauma exposure. Moreover, the trajectory of self-reported PTSD symptom severity was associated with projected end-state PTSD symptom severity. Relationship support and depth were also found to deteriorate over time following trauma exposure. Although these trajectories were not initially associated with end-state PTSD symptom severity, these associations became significant when trauma type was controlled, such that relationship support and depth worsened over time as projected end-state PTSD symptom severity improved.

The trajectory of relationship functioning was found to vary across type of trauma exposure and type of relationship with one's close other. Relationship support and depth worsened over time for those who experienced interpersonal traumas compared with those who experienced non-interpersonal traumas. Additionally, the trajectory of relationship support, conflict, and depth was poorer for those who responded in reference to a relationship with a nonintimate close other compared with those responding in reference to a relationship with an intimate close other.

Significant associations between initial levels of interpersonal relationship functioning variables and end-state PTSD symptom also emerged. Initial relationship support was negatively associated, and initial relationship conflict was positively associated, with projected end-state PTSD symptom severity. These associations were also found to vary across type of trauma exposure and type of relationship with one's close other. Initial relationship conflict was 
positively associated with projected end-state PTSD symptom severity among participants participating with an intimate close other but not those participating with a non-intimate close other. Moreover, there was a negative association between initial relationship support and projected end-state PTSD symptom severity among those who experienced non-interpersonal traumas, and a positive association between initial relationship depth and projected end-state PTSD symptom severity among those who experienced interpersonal traumas.

\section{Trajectory of PTSD Following Trauma Exposure: Aim 1}

The trajectory of PTSD symptomatology over time in the current sample was consistent with the existing conceptualization of PTSD as a disorder of impeded versus natural recovery (Blanchard, Hickling et al., 1996; Riggs et al., 1995; Rothbaum et al., 1992). As hypothesized, both self-reported and clinician-assessed PTSD symptom severity declined over time within the overall sample. Additionally, as hypothesized, there was a positive association between end-state PTSD symptom severity and the trajectory of self-reported PTSD symptom severity, indicating that declines in symptom severity occurred more rapidly as projected end-state PTSD symptom severity decreased. In contrast, and contrary to hypothesis, the trajectory of clinician-assessed PTSD symptom severity was not associated with the severity of projected end-state PTSD symptoms. Although no specific hypotheses were put forth regarding associations between initial PTSD symptom severity and end-state PTSD symptom severity, both initial clinician-assessed and self-reported PTSD symptom severity were positively associated with projected end-state PTSD symptom severity.

These results are largely consistent with well-established findings in the broader PTSD literature (e.g., Blanchard, Hickling et al., 1996; Riggs et al., 1995; Rothbaum et al., 1992; Shalev, Peri, Canetti, \& Schreiber, 1996). Previous studies have consistently found that the 
severity and prevalence of PTSD decline in the months following trauma exposure among individuals exposed to various types of traumas, including sexual assaults (Rothbaum et al., 1992), non-sexual assaults (Riggs et al., 1995), and motor vehicle accidents (Blanchard, Hickling et al., 1996). Although some studies documenting trajectories of PTSD have only followed participants for 3 months posttrauma (Riggs et al., 1995; Rothbaum et al., 1992), longer-term studies have shown that these declines can extend 1 to 2 years posttrauma (Blanchard, Hickling et al., 1996; Kessler et al., 1995).

The finding that initial PTSD symptom severity, as well as PTSD symptom trajectories differed as a function of projected-end state PTSD symptom severity is also consistent with the extant PTSD literature. For example, Rothbaum and colleagues (1992) found significantly greater initial PTSD symptom severity following trauma exposure among sexually assaulted women who met criteria for PTSD 3 months posttrauma compared with those who did not meet diagnostic criteria. This finding has been replicated in a study examining non-sexually assaulted men and women (Riggs et al., 1995). In both studies, those without end-state PTSD diagnoses made significantly greater improvements over time compared with those with end-state PTSD diagnoses. Similar findings for both initial differences in PTSD symptom severity and in symptom trajectories over 6 months have been noted in a community sample of individuals who experienced different traumatic events (Shalev et al., 1996). Finally, among motor vehicle accident survivors, remission rates over 12 months posttrauma were greater for those who were initially classified as having subsyndromal PTSD compared with those who met full diagnostic criteria; and among those initially classified as having PTSD, initial hyperarousal and avoidance symptoms were positively associated with PTSD symptom severity 12 months posttrauma (Blanchard, Hickling et al., 1996). 
Although the majority of the findings in Aim 1 were consistent with the established literature, there are some exceptions. Most notably, the trajectory of clinician-assessed PTSD symptom severity did not differ as a function of projected end-state PTSD symptom severity. This finding runs contrary to hypothesis and the established literature. The lack of a significant association may be attributable to differences in the procedure and in how data were analyzed in the current study compared with prior studies. Specifically, in the current study, participants received their first assessment an average of 124.22 days posttrauma, whereas prior studies have typically conducted their first assessments within 2 weeks of exposure (Riggs et al., 1995; Rothbaum et al., 1992; Shalev et al., 1996). Moreover, data were modeled in the current study with date of trauma exposure specified as the intercept. This was done in order to provide an estimate of PTSD symptom severity immediately after trauma exposure. However, because the first assessment occurred an average of 124.22 days posttrauma, the value of the intercept (i.e., symptom severity immediately following trauma exposure) is actually an estimate based on the trajectory of PTSD symptomatology during the posttrauma period. In contrast, prior studies have utilized the initial assessment as the indicator of initial PTSD symptom severity. It is possible that failing to capture differences in PTSD symptom severity more proximal to the trauma influenced the estimate of the trajectory of PTSD symptom severity.

Another statistically-related explanation for the lack of findings for clinician-assessed PTSD symptoms may be that previous studies have examined end-state PTSD as a predictor of symptom trajectories using a dichotomous variable to categorize participants as either meeting or not meeting diagnostic criteria for PTSD at the final assessment (e.g., Riggs et al., 1995; Rothbaum et al., 1992; Shalev et al., 1996). In contrast, the current study utilized a continuous variable to capture end-state PTSD symptom severity, to be more consistent with taxometric 
research that has shown that PTSD is better conceptualized as a dimensional construct (BromanFulks et al., 2006). Dichotomizing a continuous variable results in the loss of a significant portion of its variance, and suggests that there are only two meaningful values of the dichotomized variable. However, using a continuous variable that has been dichotomized can result in inflated associations therefore misrepresenting the effect of one variable on the other (MacCallum, Zhang, Preacher, \& Rucker, 2002).

These explanations cannot fully account for the findings of the current study, given that projected end-state PTSD symptom severity was associated with self-reported, but not clinicianassessed, PTSD symptom trajectory. One possibility that may account for this difference is that, during the administration of the clinician-administered interview, assessors explicitly instructed participants to only endorse symptoms present within the reporting period (i.e., the 30 days prior to the given assessment). Participants routinely needed to be reminded of the reporting period in the interviews, because they often reported on symptoms they had experienced in the initial weeks or month after the trauma occurred. Although the self-report measure of PTSD asked participants to report on their symptomatology over the past month, it is possible they were less adherent to the specific reporting period, therefore reporting on the severity of their symptoms closer to the trauma. This, in turn, may have influenced the trajectory of self-reported PTSD symptoms, leading to different trajectories of self-reported PTSD symptoms across different severities of projected end-state PTSD.

With the exception of a null finding for an association between the trajectory of clinicianassessed PTSD and projected end-state PTSD symptom severity, findings of the analyses in Aim 1 are consistent with those in the broader PTSD literature. This demonstrates that the manifestation of PTSD symptomatology displayed by the sample in the current study was similar 
to that which has been established in other samples. When investigating the trajectory of other variables during the posttrauma period, specifically those with a less established literature base (e.g., interpersonal relationship functioning), it is important to ensure that the sample is representative of those typically found in the literature, in order to help rule out the possibility that sample specific factors influenced study outcomes. Accordingly, given that the current study had a focus on investigating the trajectories of interpersonal relationship functioning variables in the posttrauma period, ensuring that the current sample was comparable to samples in previous studies allowed for greater confidence in the results of the proceeding aims (Aims 2, 3, and 4).

\section{Trajectory of Interpersonal Relationship Functioning Following Trauma Exposure: Aims 2}

and 3

The second and third aims of the current study focused on examining trajectories of interpersonal relationship functioning following trauma exposure, as well as the effects of endstate PTSD, trauma-type (interpersonal vs. non-interpersonal), and type of close significant other (intimate vs. non-intimate) on those trajectories. The goals of the analyses in Aims 2 and 3 were to establish an understanding of how interpersonal relationship functioning variables may change following trauma exposure and identify factors that may influence that change. In the overall sample, relationship support and depth were found to deteriorate following trauma exposure. Interestingly, none of the trajectories of the interpersonal relationship functioning variables were associated with end-state PTSD symptom severity.

Though there has been substantial research examining the trajectory of PTSD following trauma exposure (e.g., Blanchard, Hickling et al., 1996; Riggs et al., 1995; Rothbaum et al., 1992; Shalev et al., 1996), and the association between interpersonal relationship functioning and PTSD more generally (see Taft et al., 2011), there has been a relative paucity of research 
examining the trajectory of interpersonal relationship functioning following trauma exposure. The few studies that have investigated the effect of trauma exposure on interpersonal relationship functioning suggest that these effects are largely accounted for by PTSD symptomatology (Gimbel \& Booth, 1994; Orcutt, King, \& King, 2003; Taft, Schumm, Panuzio, \& Proctor, 2008). However, it is worth noting that all of the extant studies examined veteran samples and defined trauma exposure as "combat exposure" or "war-zone stress". Additionally, only one of them used a longitudinal design and included both male and female veterans (Taft et al., 2008), whereas the others used cross-sectional data analyses of male veterans (Gimbel \& Booth, 1994; Orcutt et al., 2003).

The current study is the first to investigate changes in facets of interpersonal relationship functioning following trauma exposure in a community sample exposed to various types of traumatic events. Accordingly, it aimed to fill an important gap in the literature about the associations between trauma exposure, PTSD, and relationship functioning in a broader traumaexposed population. Given that significant declines in relationship support and depth were detected during the posttrauma period, it appears that exposure to trauma can result in the deterioration of positive aspects of relationship functioning.

As noted above, prior veteran studies have suggested that PTSD symptomatology, as opposed to trauma exposure, accounts for changes in relationship functioning (Gimbel \& Booth, 1994; Orcutt et al., 2003; Taft et al., 2008). Accordingly, one would suspect that the inclusion of PTSD symptomatology in the models would likely account for interpersonal relationship functioning. However, the findings of the current study were inconsistent with this conclusion, as the trajectories of the interpersonal relationship functioning variables were not associated with projected end-state PTSD symptom severity. This may be attributable to heterogeneity in the 
sample of the current study that occurred as a result of including individuals exposed to various types of traumas and who were participating with different types of close others. For example, there is well established research demonstrating different prevalence rates or risk for PTSD following exposure to different types of traumatic events, with greater risk following interpersonal trauma (Breslau et al., 1998; Frans et al., 2005; Kessler et al., 1995). Accordingly, there may have been systematic differences in PTSD symptom manifestation based on type of trauma, which influenced the results. Interestingly, when the effect of trauma type was controlled in the respective models (i.e., the effect of trauma type on the trajectory of relationship functioning), relationship support and depth worsened over time as projected end-state PTSD symptom severity improved. This finding suggests a possible suppressor effect (Ludlow \& Klein, 2014), such that the addition of trauma type to the model accounted for some of the variance in end-state PTSD, clarifying, and therefore strengthening, the association between end-state PTSD symptom severity and the slope of relationship support and depth. In essence, the addition of trauma type helped account for the heterogeneity in end-state PTSD across different types of traumas.

Contrary to expectation, with inclusion of trauma type in the models, the trajectory of relationship support and depth declined as end-state PTSD symptom severity improved. A possible interpretation of these findings is that there may have been an immediate rise in relationship support and depth following trauma exposure (reflected in the initial assessment), which then regressed for that relationship as PTSD symptoms remitted (reflected in subsequent assessment). It is important to remember that participants were required to enroll with a close significant other who was aware of the trauma. Thus, it is possible that the act of disclosing the occurrence of trauma resulted in this immediate increase of support and depth from participating 
close significant others. This is consistent with research suggesting that the relational benefits of self-disclosure occur through the recipient's response to disclosure as opposed to self-disclosure in and of itself (Greene, Derlega, \& Mathews, 2006). Moreover, there is the possibility of a selection bias as a result of only having close significant others who were willing to participate in the study. These close others may be qualitatively more supportive and attuned to the needs of trauma-exposed participants compared with close others who were unwilling to participate following disclosure or traumatized others unwilling to disclose to their close other. In any of these cases, the immediate rise in support and depth may be protective with regard to PTSD and may promote a trajectory of recovery, which is consistent with what is known about social support as a risk/resilience factor for PTSD (Brewin et al., 2000; Ozer et al., 2003).

The trajectory of relationship functioning was also found to vary across type of trauma exposure and type of relationship with one's close other. Specifically, relationship support and depth worsened over time for those who experienced interpersonal traumas compared with those who experienced non-interpersonal traumas. Given that interpersonal traumas are more likely to lead to a diagnosis of PTSD (i.e., Breslau et al., 1998; Frans et al., 2005; Kessler et al., 1995) and that PTSD is associated with poorer relationship functioning (Taft et al., 2011), it is likely that the association between trauma type and the trajectory of relationship support and depth occurred, at least in part, through the presence and severity of PTSD symptomatology. Additionally, the trajectory of relationship support, conflict, and depth was poorer for those who responded in reference to a relationship with a non-intimate close other compared with those responding in reference to a relationship with an intimate close other.

Contrary to hypothesis, the association between end-state PTSD and the trajectory of relationship functioning over time did not differ as a function of either trauma type or type of 
close significant other. Given the heterogeneity of the current sample with regard to trauma type and type of close significant other, it is possible that either model alone (i.e., the model examining the time by trauma type by end-state PTSD interaction and the model examining the time by close other type by end-state PTSD interaction) were insufficient to fully account for the trajectory of interpersonal relationship functioning following trauma exposure. Instead, the inclusion of all of these variables (i.e., time, trauma type, close other type, and end-state PTSD) and their corresponding two-way, three-way, and four-way interaction terms within a single model may have more fully depicted the trajectories of interest. Notably, a larger sample would be needed to adequately power and detect these interactions.

\section{Associations between Initial Levels of Interpersonal Relationship Functioning and Projected End-state PTSD Symptom Severity: Aims 2 and 3}

The current study builds on the understanding of the influence of interpersonal relationship functioning around the time of trauma exposure as risk and/or resilience factors for PTSD. Consistent with previous literature (Brewin et al., 2000; Ozer et al., 2003), there was a significant negative association between initial relationship support and projected end-state PTSD symptom severity. In addition, initial relationship conflict was positively associated with projected end-state PTSD symptom severity. Although the protective effects of social support have been well documented, the risk for PTSD that relationship conflict may confer is more novel, and expands on the current understanding of interpersonal variables associated with PTSD.

When the type of close other by end-state PTSD symptom severity interaction was added to the model examining initial relationship conflict, the main effect of end-state PTSD became non-significant and a significant interaction was detected. Initial relationship conflict was 
positively associated with projected end-state PTSD symptom severity among participants participating with an intimate close other but not those participating with a non-intimate close other. Although prior work has demonstrated a cross-sectional association between family conflict and PTSD among children (i.e., among non-intimate relationships; e.g., Bokszczanin, 2008), it seems that the association between PTSD and relationship functioning is strongest for intimate relationships.

The current study also provides a more fine-grained understanding of the role of interpersonal relationship functioning variables in risk/resilience for PTSD, by examining the effects of the trauma type by projected end-state PTSD interaction and the close other type by projected end-state PTSD interaction on initial relationship functioning. On average, initial levels of relationship functioning did not differ by type of trauma; as one would not expect relationship quality to influence exposure to specific types of trauma. However, consistent with previous research on different types of relationships (e.g., Beach, Martin, Blum, \& Roman, 1993), those who responded in reference to an intimate close other reported significantly greater depth and support in their relationship compared with those who responded in reference to a non-intimate close other.

The significant interactions between trauma type and projected end-state PTSD present a more nuanced understanding of the role of interpersonal relationship functioning in risk/resilience for PTSD. Specifically, there was a negative association between initial relationship support and projected end-state PTSD symptom severity among those who experienced non-interpersonal traumas. In contrast, there was a positive association between initial relationship depth and projected end-state PTSD symptom severity among those who experienced interpersonal traumas. The differences in these associations might be understood by 
considering that interpersonal traumas present an increased risk for PTSD compared with noninterpersonal trauma (Breslau et al., 1998; Frans et al., 2005; Kessler et al., 1995). In this vein, it may be that trauma type was a proxy for risk of PTSD within the current study. Accordingly, different aspects of interpersonal relationship functioning may influence risk/resilience for PTSD when overall risk for PTSD, based on exposure to specific types of trauma, is either high or low. More specifically, social support may be a risk/resilience factor for PTSD for traumas with a lower risk for PTSD (e.g., accidents), but not traumas with a higher risk for PTSD (e.g., sexual or physical assaults). This interpretation suggests that the buffering effect of social support is insufficient to overcome the risk conferred by exposure to traumas with a higher risk for PTSD, or that the detrimental effect of poor social support does not confer additional risk beyond that attributable to exposure to traumas with a higher risk of PTSD. It is worth noting that this interpretation may seem contrary to prior work that has shown a greater association between social support and PTSD among military samples (typically with PTSD secondary to combat; a trauma with higher risk for PTSD) compared with civilian samples (with PTSD secondary to mixed traumas; Brewin et al., 2000). However, the civilian category in this prior work aggregated across various samples with exposure to different types of traumas, including interpersonal and non-interpersonal traumas, (e.g., disaster, crime, motor vehicle accidents). Thus, it does not directly address differences in associations between social support and PTSD across low- and high-risk traumas.

Although the interaction between trauma type and end-state PTSD symptom severity was in the opposite direction for relationship depth compared with relationship support, the notion of considering the base rate for PTSD across different types of traumas might be applied. Whereas relationship support appears to confer its risk/resilience among low-risk traumas, relationship 
depth appears to confer its risk/resilience among high-risk traumas. Individuals who have a relationship with their close significant other that is high in depth may have a close other who feels more responsible for the traumatized individual's well being. One potential mechanism for this association is that the close other may engage in accommodating behaviours, in an effort to ease the trauma-exposed individual's distress, (Fredman, Vorstenbosch, Wagner, Macdonald, \& Monson, 2014). This, in turn, may create an environment that prevents opportunities for the trauma-exposed individual to approach situations that serve to disconfirm trauma-related beliefs and aid in the recovery process. This explanation is partially supported by preliminary longitudinal work demonstrating a positive association between early accommodation from female intimate partners and later situational avoidance of male service members (Campbell, Renshaw, Kashdan, Curby, \& Carter, submitted). Alternatively, individuals with deeper relationships may have close significant others who are more comfortable being critical or hostile (Tarrier, 1996), which in turn may interfere with the recovery process. The detrimental effect of high relationship depth, appears only to occur for those exposed to traumas associated with a higher risk of PTSD, whereas it does not confer additional risk for traumas with lower risk of PTSD.

\section{Directionality of the Association between PTSD and Interpersonal Relationship Functioning Following Trauma Exposure: Aim 4}

The final aim of the current study was to investigate the directionality of associations between PTSD symptom severity and interpersonal relationship functioning following trauma exposure. Although it was hypothesized that interpersonal relationship functioning would significantly predict changes in PTSD symptom severity within the first year of exposure, and that PTSD symptom severity would significantly predict changes in interpersonal relationship 
functioning beyond one year post-exposure, none of the cross-lagged effects (i.e., the effect of PTSD at one time point on changes in interpersonal relationship functioning from that time point to the next, and vice versa) were significant.

The current study was designed with limited inclusion/exclusion criteria in order to increase its external validity. Although this can be viewed as a strength, it may have contributed to the lack of findings for analyses in Aim 4. More specifically, methodological differences between the current and previous studies may explain the lack of findings. For example, the current study recruited a large community sample exposed to a wide range of traumas. In contrast, previous studies have exclusively looked at specific types of traumas, the majority of which have been deployment or combat-related traumas (e.g., Erbes et al., 2012; King, Taft et al., 2006; Laffaye et al., 2008; Skopp et al., 2011). Additionally, although the current study evaluated interpersonal relationship functioning across an array of relationships (e.g., the participant's close significant other could have been an intimate partner, family member, or close friend), it was limited in that the analyses in Aim 4 were not conducted separately for each close other type, due to the relatively large sample size needed to conduct these analyses. In contrast, previous studies have examined interpersonal relationship functioning in reference to the same type of relationship for the entire sample (e.g., intimate relationship adjustment; Erbes et al., 2012; support from family; Kaniasty \& Norris, 2008) or have used a more global assessment of interpersonal relationship functioning not linked to a specific person or relationship type (e.g., perceived social support more generally; King, Taft et al., 2006; Perry et al., 1992). The lack of findings in the current study, coupled with significant finding in previous studies, suggest that there may be important differences associated with the type of trauma exposure and type of relationship that were obscured. Consistent with this interpretation, some meta-analytic studies 
support the notion that the strength of the relationship between interpersonal relationship functioning and PTSD differs as a function of trauma type, with a stronger association between interpersonal variables and PTSD within military populations compared with civilian populations (Brewin et al., 2000; Taft et al., 2011).

\section{Limitations}

Although the current study has several strengths, it is important to note its limitations. For instance, the wide enrollment window (i.e., up to 6 months posttrauma exposure), and corresponding average number of days between trauma exposure and the first assessment (i.e., 124.22 days), may have limited the ability to detect important shorter-term changes in PTSD and interpersonal relationship functioning. A 6-month enrollment window was utilized to balance the feasibility of recruiting a trauma-exposed sample with capturing the trajectory of recovery following trauma exposure. However, previous work has demonstrated that a large amount of change in PTSD symptomatology occurs within 3 months following trauma exposure (Riggs et al., 1995; Rothbaum et al., 1992), and thus the current study may have missed this change that may be important when examining the trajectory of interpersonal relationship functioning and the direction of its association with PTSD over time. Relatedly, the analyses utilized in the current study modeled the intercept terms (e.g., initial status) for PTSD and interpersonal relationship functioning variables by using the trajectory of each variable. Although this in and of itself is not problematic, the relative lack of data between trauma exposure and the first assessment (i.e., roughly 4 months), coupled with the possibility that early changes in these variables were missed, may have biased the estimates of these intercept terms.

As noted earlier, the current study is also limited by the heterogeneity of its sample with regard to trauma exposure and relationship type. Though this can be considered a strength of the 
study with regard to the generalizability of findings, the study may have benefitted from narrower inclusion criteria (e.g., limiting the type of traumas permitted for enrollment, restricting enrollment to only participants who included intimate close others). Narrower inclusion/exclusion criteria may have been useful in understanding the interplay between PTSD and interpersonal relationship functioning within more specific samples (e.g., among individuals exposed to sexual violence and their intimate partners). An alternative approach could have been to recruit a larger number of participants in order to account for the potential moderating effects of these variables.

Although the assessment of PTSD was multimodal (i.e., it included both self-report and clinician-rated measures), the measure of interpersonal relationship functioning was limited to self-report only. The study could have benefited from a more thorough assessment of interpersonal relationship functioning, including clinician assessment and self-report from one's the close significant other. Similarly, data regarding interpersonal relationship functioning prior to trauma exposure may also have been helpful to more clearly understand the effects of trauma on relationships. Although there were benefits of utilizing of the QRI, including its applicability to a wide range of relationships, there were limitations associated with its use. Specifically, the small number of items in each subscale limited the variability of each construct and thus may have made it difficult to detect differences in interpersonal relationship functioning across participants, as well as changes in these constructs over time.

Additionally, the current study is limited because it did not account for factors that may have influenced the association between interpersonal relationship functioning and PTSD. For example, the current study did not control for patients' prior trauma histories and associated preexisting PTSD symptoms. Similarly, it did not control for other mental health conditions, which 
have well-documented associations with interpersonal relationship functioning (e.g., depression, alcohol dependence, generalized anxiety disorder; Whisman, 1999; Whisman, Sheldon, \& Goering, 2000). Finally, it did not control for potential treatment effects; there may have been differential effects of interpersonal relationship functioning on PTSD, and vice versa, for individuals who were receiving treatment compared with those who were not in treatment.

\section{Future Directions}

Despite its limitations, the current study provides a valuable contribution to the literature and highlights a number of important areas for future work. Primarily, future studies could benefit from replicating this work, either in more specific samples (e.g., among participants exposed to specific types of trauma; among only intimate relationships), or by controlling for these factors in larger and more diverse samples. Other mental health conditions and factors that may influence the association between PTSD and interpersonal relationship functioning should also be considered. Future studies should also make efforts to recruit participants more proximal to the trauma, in order to prevent the loss of potential critical information during the early weeks posttrauma. Recent studies have begun to test the feasibility of, and develop a framework for, recruitment in emergency rooms immediately after exposure to trauma (Malcoun et al., 2010; Rothbaum et al., 2008, 2012). However, it will be important to consider how to recruit participants who do not present to the emergency room after trauma exposure.

Multimodal assessment of interpersonal relationship functioning should also be considered when expanding on this work. In addition to the use of self-report measures, clinician-administered and significant other-report measures should be utilized in order to ensure a comprehensive depiction of interpersonal relationship functioning. Similarly, the inclusion of significant others' assessment of participants' PTSD symptoms may also be informative in 
understanding the association between PTSD and interpersonal relationship functioning, because previous studies have documented that partner perceptions of PTSD symptoms can be associated with relationship distress (Renshaw \& Campbell, 2011).

Finally, future studies should consider examining specific PTSD symptom clusters individually, rather than examining PTSD as a single construct. There is evidence to suggest that the emotional numbing and hyperarousal symptom clusters of PTSD in the DSM-IV-TR (American Psychiatric Association, 2000), now captured in the negative alterations in cognition and mood and alterations in arousal and reactivity clusters in the fifth edition of the Diagnostic and Statistical Manual of Mental Disorders (DSM-5; American Psychiatric Association, 2013), are most closely linked to impaired relationship functioning (Cook, Riggs, Thompson, Coyne, \& Sheikh, 2004; Evans, McHugh, Hopwood, \& Watt, 2003; Riggs, Byrne, Weathers, \& Litz, 1988; Taft et al., 2008). More specifically, it has been proposed that emotional numbing may influence relationship quality through deficits in communication and emotional connection, whereas hyperarousal may exert its effects via anger and aggression (for a review, see Renshaw et al., 2011). Thus, evaluating symptom clusters individually may provide a more nuanced understanding of how specific domains of interpersonal relationship functioning influence specific PTSD symptom clusters, and vice versa, following trauma exposure.

\section{Conclusion and Clinical Implications}

The current study was designed to advance the understanding of PTSD and interpersonal relationships functioning. Although many of the hypothesized outcomes were not found, the results can still be used to inform early intervention efforts aimed at reducing risk for PTSD following trauma exposure. Specifically, given the associations between interpersonal relationship functioning and end-state PTSD, brief interpersonally-based interventions should be 
considered following trauma exposure. These interventions might be "indicated" based on the type of trauma to which one is exposed and whether someone is in an intimate relationship. More specifically, interventions should aim to increase social support following exposure to traumas that have a lower risk for PTSD (e.g., motor vehicle accidents). In contrast, interventions that aim to educate close significant others about PTSD symptom accommodation and ensure that trauma exposed individuals are provided with opportunities to approach trauma-related stimuli may be particularly beneficial among those exposed to traumas with a higher risk of PTSD. Finally, among trauma-exposed individuals in intimate relationships, interventions should aim to decrease relationship conflict via dyadic conflict management strategies and couple communication skills training. Although further research is needed to test the feasibility and efficacy of interpersonally-based early interventions following trauma exposure, they may be provide an avenue for promoting posttraumatic recovery, prevent chronic PTSD, and reducing the individual and societal costs associated with this condition. 


\section{References}

Akaike, H. (1973). Information theory as an extension of the maximum likelihood principle. In B. N. Petrov, \& F. Csaki (Eds.), Second International Symposium on Information Theory (pp. 267-281). Akademiai Kiado: Budapest.

Allen, E. S., Rhoades, G. K., Stanley, S. M., \& Markman, H. J. (2010). Hitting home:

Relationships between recent deployment, posttraumatic stress symptoms, and marital functioning for Army couples. Journal of Family Psychology, 24, 280-288. doi: $10.1037 / \mathrm{a} 0019405$

American Psychiatric Association. (2000). Diagnostic and statistical manual of mental disorders (4th ed., text rev.). Washington, DC: Author.

American Psychiatric Association. (2013). Diagnostic and statistical manual of mental disorders (5th ed.). Washington, DC: Author.

Beach, S. R., Martin, J. K., Blum, T. C., \& Roman, P. M. (1993). Effects of marital and coworker relationships on negative affect: Testing the central role of marriage. American Journal of Family Therapy, 21, 313-323. doi: 10.1080/01926189308251002

Bentler, P. M. (1990). Comparative fit indexes in structural models. Psychological Bulletin, 107, 238-246. doi: 10.1037/0033-2909.107.2.238

Berscheid, E., Snyder, M., \& Omoto, A. M. (1989). The Relationship Closeness Inventory: Assessing the closeness of interpersonal relationships. Journal of Personality and Social Psychology, 57, 792-807. doi: 10.1037//0022-3514.57.5.792

Blake, D. D., Weathers, F. W., Nagy, L. M., Kaloupek, D. G., Gusman, F. D., Charney, D. S., \& Keane, T. M. (1995). The development of a clinician-administered PTSD scale. Journal of Traumatic Stress, 8, 75-90. doi: 10.1002/jts.2490080106 
Blanchard, E. B., Hickling, E. J., Barton, K. A., Taylor, A. E., Loos, W. R., \& Jones-Alexander, J. (1996). One-year prospective follow-up of motor vehicle accident victims. Behaviour Research and Therapy, 34, 775-786. doi: 10.1016/0005-7967(96)00038-1

Blanchard, E. B., Jones-Alexander, J., Buckley, T. C., \& Forneris, C. A. (1996). Psychometric properties of the PTSD Checklist (PCL). Behaviour Research and Therapy, 34, 669-673. doi: 10.1016/0005-7967(96)00033-2

Blunch, N. J. (2008). Introduction to structural equation modeling using SPSS and AMOS. Thousand Oaks, CA: Sage.

Bokszczanin, A. (2008). Parental support, family conflict, and overprotectiveness: Predicting PTSD symptom levels of adolescents 28 months after a natural disaster. Anxiety, Stress, \& Coping, 21), 325-335. doi: 10.1080/10615800801950584

Breslau, N., Kessler, R. C., Chilcoat, H. D., Schultz, L. R., Davis, G. C., \& Andreski, P. (1998). Trauma and posttraumatic stress disorder in the community: The 1996 Detroit area survey of trauma. Archives of General Psychiatry, 55, 626-632. doi: 10.1001/archpsyc.55.7.626

Brewin, C. R., Andrews, B., \& Valentine, J. D. (2000). Meta-analysis of risk factors for posttraumatic stress disorder in trauma-exposed adults. Journal of Consulting and Clinical Psychology, 68, 748-766. doi: 10.1037//0022-006X.68.5.748

Broman-Fulks, J. J., Ruggiero, K. J., Green, B. A., \& Kilpatrick, D. G. (2006). Taxometric investigation of PTSD: Data from two nationally representative samples. Behavior Therapy, 37, 364-380. doi: 10.1016/j.beth.2006.02.006

Campbell, Renshaw, Kashdan, Curby, \& Carter. A daily diary study of PTSD and romantic partner accommodation. Manuscript submitted for publication. 
Cook, J. M., Riggs, D. S., Thompson, R., Coyne, J. C., \& Sheikh, J. I. (2004). Posttraumatic stress disorder and current relationship functioning among World War II ex-prisoners of war. Journal of Family Psychology, 18, 36-45. doi:10.1037/0893-3200.18.1.36

Erbes, C. R., Meis, L. A., Polusny, M. A., \& Compton, J. S. (2011). Couple adjustment and posttraumatic stress disorder symptoms in National Guard veterans of the Iraq war. Journal of Family Psychology, 25, 479-487. doi: 10.1037/a0024007

Erbes, C. R., Meis, L. A., Polusny, M. A., Compton, J. S., \& Wadsworth, S. M. (2012). An examination of PTSD symptoms and relationship functioning in US soldiers of the Iraq War over time. Journal of Traumatic Stress, 25, 187-190. doi: 10.1002/jts.21689

Evans, L., McHugh, T., Hopwood, M., \& Watt, C. (2003). Chronic posttraumatic stress disorder and family functioning of Vietnam veterans and their partners. Australian and New Zealand Journal of Psychiatry, 37, 765-772. doi: 10.1080/j.1440-1614.2003.01267.x

Foa, E. B., \& Tolin, D. F. (2000). Comparison of the PTSD Symptom Scale-Interview Version and the Clinician-Administered PTSD Scale. Journal of Traumatic Stress, 13, 181-191. doi: 10.1023/A:1007781909213

Frans, Ö., Rimmö, P. A., Åberg, L., \& Fredrikson, M. (2005). Trauma exposure and posttraumatic stress disorder in the general population. Acta Psychiatrica Scandinavica, 111, 291-290. doi: 10.1111/j.1600-0447.2004.00463.x

Fredman, S. J., Vorstenbosch, V., Wagner, A. C., Macdonald, A., \& Monson, C. M. (2014). Partner accommodation in posttraumatic stress disorder: Initial testing of the Significant Others' Responses to Trauma Scale (SORTS). Journal of Anxiety Disorders, 28, 372381. doi: 10.1016/j.janxdis.2014.04.001

Galovski, T., \& Lyons, J. A. (2004). Psychological sequelae of combat violence: A review of the 
impact of PTSD on the veteran's family and possible interventions. Aggression and Violent Behavior, 9, 477-501. doi: 10.1016/S1359-1789(03)00045-4

Gimbel, C., \& Booth, A. (1994). Why does military combat experience adversely affect marital relations? Journal of Marriage and the Family, 56, 691-703. doi: 10.2307/352879

Greene, K., Derlega, V. J., \& Mathews, A. (2006). Self-disclosure in personal relationships. In A. L. Vangelisti \& D. Perlman (Eds.), The Cambridge handbook of personal relationships (pp. 409-427). Cambridge, UK: Cambridge University Press.

Griffin, M. A. (1997). Interaction between individuals and situations: Using HLM procedures to estimate reciprocal relationships. Journal of Management, 23, 759-773. doi: $10.1177 / 014920639702300604$

Hamagami, F., \& McArdle, J. J. (2001). Advanced studies of individual differences linear dynamic models for longitudinal data analysis. In G. Marcoulides \& R. Schumacker (Eds.), New developments and techniques in structural equation modeling (pp. 203-246). Hillside, NJ: Erlbaum.

Hovens, J. E., Van der Ploeg, H. M., Klaarenbeek, M. T. A., Bramsen, I., Schreuder, J. N., \& Rivero, V. V. (1994). The assessment of posttraumatic stress disorder: with the Clinician Administered PTSD Scale: Dutch results. Journal of Clinical Psychology, 50, 325-340. doi: 10.1002/1097-4679(199405)50:3\%3C325::AID-JCLP2270500304\%3E3.0.CO;2-M

Iverson, K. M., Gradus, J. L., Resick, P. A., Suvak, M. K., Smith, K. F., \& Monson, C. M. (2011). Cognitive-behavioral therapy for PTSD and depression symptoms reduces risk for future intimate partner violence among interpersonal trauma survivors. Journal of Consulting and Clinical Psychology, 79, 193-202. doi: 10.1037/a0022512

Kaniasty, K., \& Norris, F. H. (2008). Longitudinal linkages between perceived social support 
and posttraumatic stress symptoms: Sequential roles of social causation and social selection. Journal of Traumatic Stress, 21, 274-281. doi: 10.1002/jts.20334

Kessler, R. C., Sonnega, A., Bromet, E., Hughes, M., \& Nelson, C. B. (1995). Posttraumatic stress disorder in the national comorbidity survey. Archives of General Psychiatry, 52, 1048- 1060. doi: 10.1001/archpsyc.1995.03950240066012

King, D. W., King, L. A., McArdle, J. J., Grimm, K., Jones, R. T., \& Ollendick, T. H. (2006). Characterizing time in longitudinal trauma research. Journal of Traumatic Stress, 19, 205-215. doi: 10.1002/jts.20112

King, L. A., King, D. W., McArdle, J. J., Saxe, G. N., Doron-LaMarca, S., \& Orazem, R. J. (2006). Latent difference score approach to longitudinal trauma research. Journal of Traumatic Stress, 19, 771-785. doi: 10.1002/jts.20188

King, D. W., Taft, C., King, L. A., Hammond, C., \& Stone, E. R. (2006). Directionality of the association between social support and posttraumatic stress disorder: A longitudinal investigation. Journal of Applied Social Psychology, 36, 2980-2992. doi: 10.1111/j.00219029.2006.00138.x

Laffaye, C., Cavella, S., Drescher, K., \& Rosen, C. (2008). Relationships among PTSD symptoms, social support, and support source in veterans with chronic PTSD. Journal of Traumatic Stress, 21 394-401. doi: 10.1002/jts.20348

Lambert, J. E., Engh, R., Hasbun, A., \& Holzer, J. (2012). Impact of posttraumatic stress disorder on the relationship quality and psychological distress of intimate partners: a meta-analytic review. Journal of Family Psychology, 26, 729-737. doi: $10.1037 / \mathrm{a} 0029341$

Lecrubier, Y., Sheehan, D. V., Weiller, E., Amorim, P., Sheehan, K. H., Janavs, J., \& Dunbar, G. 
C. (1997). The Mini International Neuropsychiatric Interview (MINI). A short diagnostic structured interview: Reliability and validity according to the CIDI. European Psychiatry, 12, 224-231. doi: 10.1016/S0924-9338(97)83296-8

Ludlow, L., \& Klein, K. (2014). Suppressor variables: The difference between 'is' versus 'acting as'. Journal of Statistics Education, 22.

MacCallum, R. C., Browne, M. W., \& Sugawara, H. M. (1996). Power analysis and determination of sample size for covariance structure modeling. Psychological Methods, 1, 130-149. doi: 0.1037/1082-989X.1.2.130

MacCallum, R. C., Zhang, S., Preacher, K. J., \& Rucker, D. D. (2002). On the practice of dichotomization of quantitative variables. Psychological Methods, 7, 19-40. doi: 10.1037/1082-989X.7.1.19

Malcoun, E., Houry, D., Arndt-Jordan, C., Kearns, M. C., Zimmerman, L., Hammond-Susten, M., \& Rothbaum, B. O. (2010). Feasibility of identifying eligible trauma patients for posttraumatic stress disorder intervention. Western Journal of Emergency Medicine, 11, 274-278.

McArdle, J. J. (2001). A latent difference score approach to longitudinal dynamic structural analyses. In R. Cudeck, S. DuToit, \& D. Sorbom (Eds.), Structural equation modeling: Present and future (pp. 342-380). Lincolnwood, IL: Scientific Software International.

McArdle, J. J., \& Hamagami, F. (2001). Latent difference score structural models for linear dynamic analyses with incomplete longitudinal data. In L. Collins (Ed.), New methods for the analysis of change: Decade of behavior (pp. 139-175). Washington, DC: American Psychological Association.

Melvin, K. C., Gross, D., Hayat, M. J., Jennings, B. M., \& Campbell, J. C. (2012). Couple 
functioning and post-traumatic stress symptoms in US army couples: The role of resilience. Research in Nursing \& Health, 35, 164-177. doi: 10.1002/nur.21459

Monson, C. M., Fredman, S. J., \& Dekel, R. (2010). Posttraumatic stress disorder in an interpersonal context. In J. G., Beck (Ed.), Interpersonal processes in the anxiety disorders: Implications for understanding psychopathology and treatment (pp. 179-208). Washington, DC: American Psychological Association.

Monson, C. M., Fredman, S. J., Macdonald, A., Pukay-Martin, N. D., Resick, P. A., \& Schnurr, P. P. (2012). Effect of cognitive-behavioral couple therapy for PTSD: A randomized controlled trial. Journal of the American Medical Association, 308, 700-709. doi: 10.1001/jama.2012.9307

Monson, C. M., Taft, C. T., \& Fredman, S. J. (2009). Military-related PTSD and intimate relationships: from description to theory-driven research and intervention development. Clinical psychology review, 29, 707-714. doi: 10.1016/j.cpr.2009.09.002

Moos, R. H., \& Moos, B. S. (1981). Family Environment Scale manual. Palo Alto, CA: Consulting Psychologists Press.

Nelson Goff, B. S., \& Smith, D. B. (2005). Systemic traumatic stress: The couple adaption to traumatic stress model. Journal of Marital and Family Therapy, 31, 145-157. doi: $10.1111 / \mathrm{j} .1752-0606.2005 . t b 01552 . x$

Norton, R. (1983). Measuring marital quality: A critical look at the dependent variable. Journal of Marriage and the Family, 45, 141-151. doi:10.2307/351302

Orcutt, H. K., King, L. A., \& King, D. W. (2003). Male -perpetrated violence among Vietnam veteran couples: Relationships with veteran's early life characteristics, trauma history, and PTSD symptomatology. Journal of Traumatic Stress, 16, 381-390. doi: 
10.1023/A:1024470103325

Ozer, E. J., Best, S. R., Lipsey, T. L., \& Weiss, D. S. (2003). Predictors of posttraumatic stress disorder and symptoms in adults: A meta-analysis. Psychological Bulletin, 129, 52-73. doi: $10.1037 / 0033-2909.129 .1 .52$

Perry, S., Difede, J., Musngi, G., Frances, A. J., \& Jacobsberg, L. (1992). Predictors of posttraumatic stress disorder after burn injury. American Journal of Psychiatry, 149, 931935. doi: 10.1097/00006534-199308000-00073

Pierce, G. R., Sarason, I. G., \& Sarason, B. R. (1991). General and relationship-based perceptions of social support: Are two constructs better than one? Journal of Personality and Social Psychology, 61, 1028-1039. doi: 10.1037/0022-3514.61.6.1028

Pierce, G. R., Sarason, I. G., Sarason, B. R., Solky-Butzel, J. A., \& Nagle, L. C. (1997). Assessing the quality of personal relationships. Journal of Social and Personal Relationships, 14, 339-356. doi: 10.1177/0265407597143004

Qualtrics. (2005) Qualtrics (version 55740). Provo, UT.

Raudenbush, S. W., \& Bryk, A. S. (2002). Hierarchical linear models: Application and data analysis methods. Thousand Oaks, CA: Sage.

Renshaw, K. D., Blais, R. K., \& Caska, C. M. (2011). Distress in spouses of combat veterans with PTSD: The importance of interpersonally based cognitions and behaviors. In S. MacDermid Wadsworth \& D. Riggs (eds.), Risk and resilience in U.S. military families (pp. 69-84). New York, NY: Springer.

Renshaw, K. D., \& Campbell, S. B. (2011). Combat veterans' symptoms of PTSD and partners' distress: The role of partners' perceptions of veterans' deployment experiences. Journal of Family Psychology, 25, 953-962. doi 10.1037/a0025871 
Riggs, D. S., Byrne, C. A., Weathers, F. W., \& Litz, B. T. (1998). The quality of the intimate relationships of male Vietnam veterans: Problems associated with posttraumatic stress disorder. Journal of traumatic stress, 11, 87-101. doi: 10.1023/A:1024409200155

Riggs, D. S., Rothbaum, B. O., \& Foa, E. B. (1995). A prospective examination of symptoms of posttraumatic stress disorder in victims of nonsexual assault. Journal of Interpersonal Violence, 10, 201-214. doi: 10.1177/0886260595010002005

Rothbaum, B. O., Foa, E. B., Riggs, D. S., Murdock, T., \& Walsh, W. (1992). A prospective examination of post-traumatic stress disorder in rape victims. Journal of Traumatic Stress, 5, 455-475. doi: 10.1007/BF00977239

Rothbaum, B. O., Houry, D., Heekin, M., Leiner, A. S., Daugherty, J., Smith, L. S., \& Gerardi, M. (2008). A pilot study of an exposure-based intervention in the ED designed to prevent posttraumatic stress disorder. American Journal of Emergency Medicine, 26, 326-330. doi: 10.1016/j.ajem.2007.07.006

Rothbaum, B. O., Kearns, M. C., Price, M., Malcoun, E., Davis, M., Ressler, K. J.,... Houry, D. (2012). Early intervention may prevent the development of posttraumatic stress disorder: A randomized pilot civilian study with modified prolonged exposure. Biological Psychiatry, 72, 957-963. doi: 10.1016/j.biopsych.2012.06.002

Schnurr, P. P., Friedman, M. J., Engel, C. C., Foa, E. B., Shea, M. T., Chow, B. K.,... Bernardy, N. (2007). Cognitive behavioral therapy for posttraumatic stress disorder in women: A randomized controlled trial. Journal of the American Medical Association, 297, 820-230. doi: $10.1001 /$ jama.297.8.820

Shalev, A. Y., Peri, T., Canetti, L., \& Schreiber, S. (1996). Predictors of PTSD in injured trauma survivors: A prospective study. American Journal of Psychiatry, 153, 219-225. doi: 
10.1176/ajp.153.2.219

Sheehan, D. V., Lecrubier, Y., Sheehan, K. H., Amorim, P., Janavs, J., Weiller, E.,... Dunbar, G. C. (1998). The Mini-International Neuropsychiatric Interview (MINI): the development and validation of a structured diagnostic psychiatric interview for DSM-IV and ICD-10. Journal of Clinical Psychiatry, 59, 22-33.

Singer, J. D., \& Willett, J. B. (2003). Applied longitudinal data analysis: Modeling change and event occurrence. London: Oxford University Press.

Skopp, N. A., Reger, M. A., Reger, G. M., Mishkind, M. C., Raskind, M., \& Gahm, G. A. (2011). The role of intimate relationships, appraisals of military service, and gender on the development of posttraumatic stress symptoms following Iraq deployment. Journal of Traumatic Stress, 24, 277-286. doi: 10.1002/jts.20632

Solomon, Z., Debby-Aharon, S., Zerach, G., \& Horesh, D. (2011). Marital adjustment, parental functioning, and emotional sharing in war veterans. Journal of Family Issues, 32, 127147. doi: $10.1177 / 0192513 \times 10379203$

Steiger, J. H., \& Lind, J. C. (1980, May). Statistically based tests for the number of common factors. Paper presented at the annual meeting of the Psychometric Society, Iowa City, IA.

Tabachnick, B. C., \& Fidell, L. S. (2007). Using multivariate statistics (5th ed.). Boston, MA: Allyn and Bacon.

Taft, C. T., Schumm, J. A., Panuzio, J., \& Proctor, S. P. (2008). An Examination of family adjustment among operation desert storm veterans. Journal of Consulting and Clinical Psychology, 76, 648-656. doi: 10.1037/a0012576

Taft, C. T., Watkins, L. E., Stafford, J., Street, A. E., \& Monson, C. M. (2011). Posttraumatic stress disorder and intimate relationship problems: A meta-analysis. Journal of 
Consulting and Clinical Psychology, 79, 22-33. doi: 10.1037/a0022196

Tarrier, N. (1996). An application of expressed emotion to the study of PTSD: Preliminary findings. Clinical Psychology and Psychotherapy, 3, 220-229. doi: 10.1002/(SICI)10990879(199609)3:3<220::AID-CPP104>3.0.CO;2-9

Van Ameringen, M., Mancini, C., Patterson, B., \& Boyle, M. H. (2008). Post-traumatic stress disorder in Canada. CNS Neuroscience \& Therapeutics, 14, 171-181. doi:

$10.1111 / j .1755-5949.2008 .00049 . x$

Walling, S. M., Suvak, M. K., Howard, J. M., Taft, C. T., \& Murphy, C. M. (2012).

Race/ethnicity as a predictor of change in working alliance during cognitive behavioral therapy for intimate partner violence perpetrators. Psychotherapy, 49, 180-189. doi: $10.1037 / \mathrm{a} 0025751$

Wanklyn, S. G., Pukay-Martin, N. D., Belus, J. M., St. Cyr, K., Girard, T. A., \& Monson C. M. Trauma types as differential predictors of PTSD, MDD and their comorbidity. Manuscript submitted for publication.

Weathers, F. W., Keane, T. M., \& Davidson, J. R. (2001). Clinician-Administered PTSD Scale: A review of the first ten years of research. Depression and Anxiety, 13, 132-156. doi: 10.1002/da. 1029

Weathers, F. W., Litz, B. T., Herman, J. A., Huska, J. A., \& Keane, T. M. (1993, October). The PTSD Checklist (PCL): Reliability, validity and diagnostic utility. Paper presented at the 9th annual conference of the International Society for Traumatic Stress Studies, San Antonio, TX.

Whisman, M. A. (1999). Marital dissatisfaction and psychiatric disorders: Results from the National Comorbidity Survey. Journal of Abnormal Psychology, 108, 701-706. doi: 


\subsection{7/0021-843X.108.4.701}

Whisman, M. A., Sheldon, C. T., \& Goering, P. (2000). Psychiatric disorders and dissatisfaction with social relationships: Does type of relationship matter? Journal of Abnormal Psychology, 109, 803-808. doi: 10.1037/0021-843X.109.4.803 